\title{
Hybrid Wing Body Shielding Studies Using an Ultrasonic Configurable Fan Artificial Noise Source Generating Typical Turbofan Modes
}

Daniel L. Sutliff and Clifford A. Brown

Glenn Research Center, Cleveland, Ohio

Bruce E. Walker

Channel Islands Acoustics, Camarillo, California 


\section{NASA STI Program . . . in Profile}

Since its founding, NASA has been dedicated to the advancement of aeronautics and space science. The NASA Scientific and Technical Information (STI) program plays a key part in helping NASA maintain this important role.

The NASA STI Program operates under the auspices of the Agency Chief Information Officer. It collects, organizes, provides for archiving, and disseminates NASA's STI. The NASA STI program provides access to the NASA Aeronautics and Space Database and its public interface, the NASA Technical Reports Server, thus providing one of the largest collections of aeronautical and space science STI in the world. Results are published in both non-NASA channels and by NASA in the NASA STI Report Series, which includes the following report types:

- TECHNICAL PUBLICATION. Reports of completed research or a major significant phase of research that present the results of NASA programs and include extensive data or theoretical analysis. Includes compilations of significant scientific and technical data and information deemed to be of continuing reference value. NASA counterpart of peer-reviewed formal professional papers but has less stringent limitations on manuscript length and extent of graphic presentations.

- TECHNICAL MEMORANDUM. Scientific and technical findings that are preliminary or of specialized interest, e.g., quick release reports, working papers, and bibliographies that contain minimal annotation. Does not contain extensive analysis.

- CONTRACTOR REPORT. Scientific and technical findings by NASA-sponsored contractors and grantees.
- CONFERENCE PUBLICATION. Collected papers from scientific and technical conferences, symposia, seminars, or other meetings sponsored or cosponsored by NASA.

- SPECIAL PUBLICATION. Scientific, technical, or historical information from NASA programs, projects, and missions, often concerned with subjects having substantial public interest.

- TECHNICAL TRANSLATION. Englishlanguage translations of foreign scientific and technical material pertinent to NASA's mission.

Specialized services also include creating custom thesauri, building customized databases, organizing and publishing research results.

For more information about the NASA STI program, see the following:

- Access the NASA STI program home page at http://www.sti.nasa.gov

- E-mail your question to help@sti.nasa.gov

- Fax your question to the NASA STI Information Desk at 443-757-5803

- Phone the NASA STI Information Desk at 443-757-5802

- Write to: STI Information Desk NASA Center for AeroSpace Information 7115 Standard Drive Hanover, MD 21076-1320 


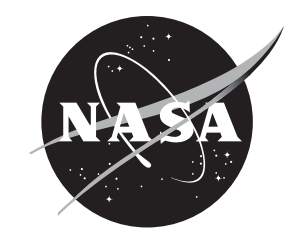

\section{Hybrid Wing Body Shielding Studies Using an Ultrasonic Configurable Fan Artificial Noise Source Generating Typical Turbofan Modes}

Daniel L. Sutliff and Clifford A. Brown

Glenn Research Center, Cleveland, Ohio

Bruce E. Walker

Channel Islands Acoustics, Camarillo, California

Prepared for

SciTech 2014

sponsored by the American Institute of Aeronautics and Astronautics

National Harbor, Maryland, January 13-17, 2014

National Aeronautics and

Space Administration

Glenn Research Center

Cleveland, Ohio 44135 


\section{Acknowledgments}

The authors world like to acknowledge the efforts of D. Podboy, L. Smith, R. Loew, B. Groening, E. Mysliwiec, and J. Mirecki of TFOME for their support in model assembly and ATL testing. C. Garcia (also TFOME) wrote the A/D and D/A algorithms used for signature generation and in-duct verification. This work was supported by the NASA Integration Systems Research Program/ Environmentally Responsible Aviation project and the Fundamental Aeronautics /Subsonic Fixed Wing Project.

Trade names and trademarks are used in this report for identification only. Their usage does not constitute an official endorsement, either expressed or implied, by the National Aeronautics and Space Administration.

This work was sponsored by the Fundamental Aeronautics Program at the NASA Glenn Research Center.

Level of Review: This material has been technically reviewed by technical management.

Available from

NASA Center for Aerospace Information 7115 Standard Drive

Hanover, MD 21076-1320
National Technical Information Service 5301 Shawnee Road Alexandria, VA 22312 


\title{
Hybrid Wing Body Shielding Studies Using an Ultrasonic Configurable Fan Artificial Noise Source Generating Typical Turbofan Modes
}

\author{
Daniel L. Sutliff and Clifford A. Brown \\ National Aeronautics and Space Administration \\ Glenn Research Center \\ Cleveland, Ohio 44135 \\ Bruce E. Walker \\ Channel Islands Acoustics \\ Camarillo, California 93010
}

\begin{abstract}
An Ultrasonic Configurable Fan Artificial Noise Source (UCFANS) was designed, built, and tested in support of the NASA Langley Research Center's 14- by 22-ft wind tunnel test of the Hybrid Wing Body (HWB) full 3-D 5.8 percent scale model. The UCFANS is a 5.8 percent rapid prototype scale model of a high-bypass turbofan engine that can generate the tonal signature of proposed engines using artificial sources (no flow). The purpose of the test was to provide an estimate of the acoustic shielding benefits possible from mounting the engine on the upper surface of an HWB aircraft using the projected signature of the engine currently proposed for the HWB. The modal structures at the rating points were generated from inlet and exhaust nacelle configurations - a flat plate model was used as the shielding surface and vertical control surfaces with correct plan form shapes were also tested to determine their additional impact on shielding. Radiated acoustic data were acquired from a traversing linear array of 13 microphones, spanning 36 in. Two planes perpendicular, and two planes parallel, to the axis of the nacelle were acquired from the array sweep. In each plane the linear array traversed four sweeps, for a total span of $168 \mathrm{in}$. acquired. The resolution of the sweep is variable, so that points closer to the model are taken at a higher resolution. Contour plots of Sound Pressure Levels, and integrated Power Levels, from nacelle alone and shielded configurations are presented in this paper; as well as the in-duct mode power levels.
\end{abstract}

\subsection{Introduction}

The Subsonic Fixed Wing (SFW) and Environmentally Responsible Aviation (ERA) Projects of NASA's Aeronautics Research Mission Directorate established research goals for the development of key technologies to a readiness level of up to six (system or subsystem prototype demonstrated in a relevant environment) by the year 2020 for the $\mathrm{N}+2$ timeframe. The subsonic $\mathrm{N}+2$ aircraft level goal was set at $42 \mathrm{~dB}$ cumulative below the Stage 4 certification level based on a study with a set of technology assumptions configured with the innovative Hybrid Wing Body (HWB) aircraft concept (Refs. 1 and 2). This study used the available shielding data from a small set of data of a point noise source shielding experiment without flow effect. In 2009, NASA and Boeing conducted a large experiment that developed the technology for jet noise shielding and, to be used for the assessment of the shielding effect of internal engine noise sources, a large database for shielding of a point noise source with flow effect (Ref. 3). This data was used in a rigorous system noise study that validated the technical approach of the earlier study to reach the $42 \mathrm{~dB}$ goal with the HWB aircraft concept (Ref. 4).

In a parallel effort, the Subsonic Fixed Wing (SFW) project began a contract with a Boeing led team in 2008 to develop improved prediction methods for shielding of engine noise that would be validated with a higher fidelity experiment to be conducted in the NASA Langley Research Center's (LaRC) 14- by 22-ft wind tunnel (Refs. 5 and 6) (see Figure 1). The N2A HWB configuration developed in this effort 


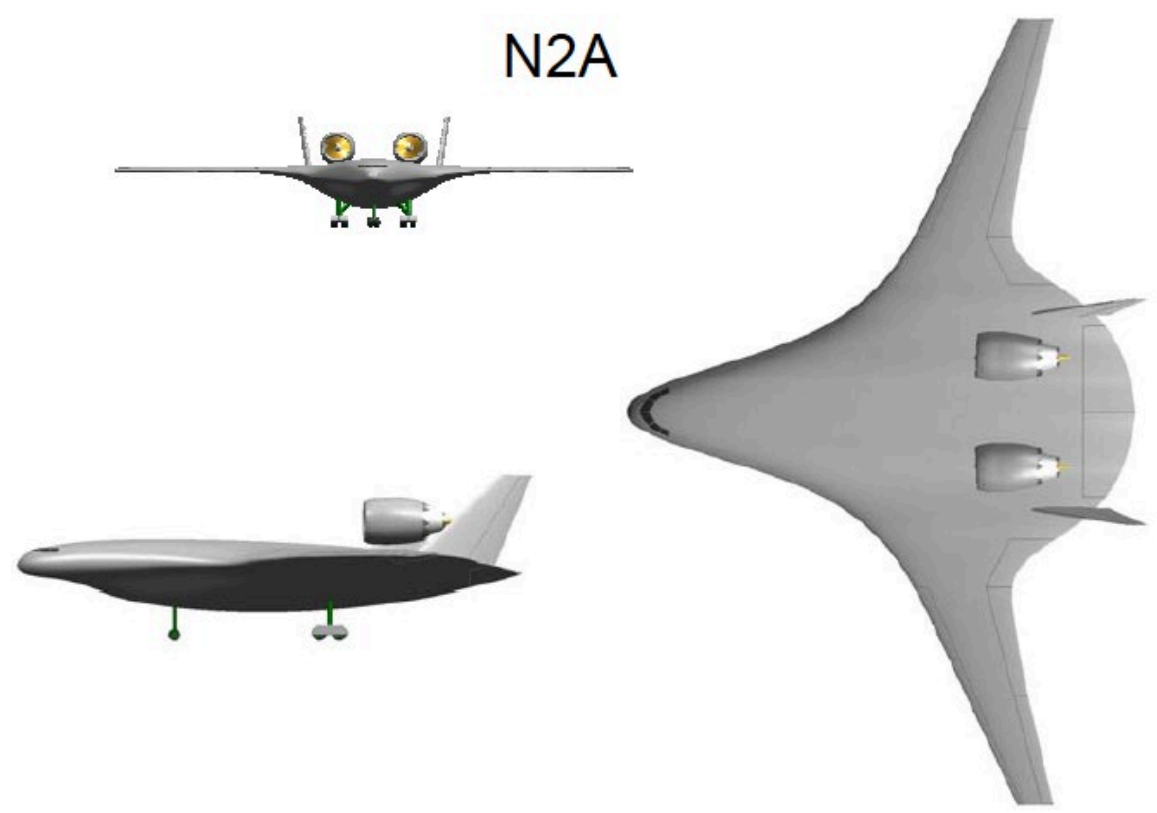

Figure 1.-A 3-D view of Hybrid Wing Body Model N2A.

was designed to meet the noise goal of $42 \mathrm{~dB}$ cumulative below Stage 4 while achieving a 25 percent fuel burn reduction compared an equivalent, current in service aircraft. NASA Aeronautics subsequently increased the fuel burn reduction goal to 50 percent, however, the N2A design was not changed because it was already in progress.

Shielding of engine noise by airframe components is a significant consideration in the design of nextgeneration transport aircraft to meet these goals. Fan tones and broadband noise are important components of engine noise. Tones in particular tend to be characterized by discrete radiated modes with distinctive radiation patterns. Modeling aircraft noise accurately will depend partially on knowledge of the effect of nearby barriers on these radiation patterns. The Ultrasonic Configurable Fan Artificial Noise Source (UCFANS) is part of the larger effort mentioned above and was developed to provide a higher fidelity noise source compared to the broadband point noise source used in the earlier shielding studies and, therefore, more representative of realistic fan noise sources. The purpose of this paper is the documentation of a scalemode test nacelle to validate code predictions of shielding and scattering of modal radiation from realistic representations of turbofan inlets and exhausts when located near finite barrier surfaces.

The test described in this paper is in support of the HWB 5.8 percent scale model test at LaRC's 14by $22-\mathrm{ft}$ wind tunnel. The HWB test will be conducted to determine the noise characteristics of the N2A HWB aircraft and in particular to determine the acoustic shielding benefits from mounting the engines on the upper surface of the airframe. Jet noise as well as the broadband component of turbo-machinery noise was simulated. The purpose of the UCFANS test is to provide complementary data on the airframe interaction of the tonal component of turbo-machinery noise. The data will be used to estimate shielding from the HWB configuration and to provide a database for shielding code validation.

The UCFANS is a 5.8 percent scale model of a turbofan nacelle and fan duct currently predicted for the HWB. It was designed, built, and tested for measuring acoustic shielding by prospective airframe components of modal fan tone radiation in an anechoic chamber. Artificial (no flow) noise sources are used in the model to reproduce the noise characteristics of a turbofan engine without the complexities of scaling down an operational fan. The artificial sources also offer additional control over the mode and frequency at each point to give a more precise database for prediction code development and validation. Model fabrication was accomplished using rapid-prototype technology at NASA Glenn Research Center (GRC).

An array of 36 wide-bandwidth electrostatic actuators was installed in a dual annulus within the fan duct and driven with modally phased tone signals between 7 and $40 \mathrm{kHz}$. Three rows of 24 widebandwidth microphones were installed in the duct between the actuator array and the configurable 
inlet/exhaust exit plane to measure the modal tone generation. Modal excitation and analysis at up to nine simultaneous frequencies was accomplished by multiplexing. Note that this arrangement allows fine control over tone frequency and azimuthal mode but only limited control over radial modes.

Spectral components of the in-duct microphone data corresponding to reference excitation frequencies were spatially filtered to recover complex amplitudes of circumferential mode orders for each of the three rings. For each circumferential mode, radial components were estimated by steering vector matrix inversion for the three rings. Far-field radiation was measured using a 3-D traversing microphone rake. Corresponding far-field data was converted to "lossless" (spherical spreading only) levels at the locations of the microphones based on temperature and humidity data recorded for each data set.

An earlier paper (Ref. 7) documented the results from using simpler modes more suitable for code validation and provided limited repeatability and signal-to-noise ratios.

\subsection{Facility}

The Acoustical Testing Laboratory (ATL) (Ref. 8) consists of a 23-by 27 - by 20 -ft (height) convertible hemi/anechoic chamber and separate sound-attenuating test support enclosure. Absorptive 34-in. fiberglass wedges in the test chamber provide an anechoic environment down to $100 \mathrm{~Hz}$. A springisolated floor system affords vibration isolation above $3 \mathrm{~Hz}$. These criteria, along with very low design background levels, enable the acquisition of accurate and repeatable acoustical measurements on test articles that radiate very little noise. Removable floor wedges allow the test chamber to operate in either a hemi-anechoic or anechoic configuration, depending on the size of the test article and the specific test being conducted (the UCFANS test was conducted in the anechoic configuration). The test support enclosure functions as a control room during normal operations.

Acoustic data were acquired from an array of 13, 1/4 in. condenser style microphones. These microphones were mounted on a linear array, spaced 3 in. apart (resulting in a 36 in. span). A traverse system was utilized to move the linear array throughout the test chamber. This traverse was limited to planar motion, (e.g., a horizontal or a vertical plane, but no arcs).

\subsection{Test Articles}

The UCFANS test article was based on the 5.8 percent scale model of the proposed nacelle of the N2A-EXTE HWB model. This scale factor resulted in a duct diameter of approximately 6-in., and a nacelle length of approximately 1 -ft. At this scale factor the approximate full-scale relevant frequency range scaled from 400 to $4,000 \mathrm{~Hz}$ is $\sim 7$ to $70 \mathrm{kHz}$. The cut-on circumferential mode generation for the proposed N2A turbofan, based on rotor blade and stator vane count, is $m=10$ at 2 BPF and $m=-8$ at $3 \mathrm{BPF}$. These approximate parameters were used to guide the UCFANS design process. The full acoustic signature definition of the engine proposed for the N2A is provided in Section 4.1.

The model was manufactured at GRC using rapid prototyping methods. The 'wing' used to determine the shielded radiation was a $2-\mathrm{D} 1 / 4 \mathrm{in}$. thick aluminum plate. The trailing and leading edge was manufactured to match the contours of the HWB N2A model, but the wing plan form was not. The edges were then faired back from a point where to meet N2A contours to the $1 / 4 \mathrm{in}$. plate. This was deemed an acceptable change to the 3-D contour as the primary impact to scattering is concentrated near the edge, and the projected area contributes mostly to shielding.

\subsection{Actuators}

The frequency range from blade passing frequency (BPF) at approach to $3 \mathrm{BPF}$ at takeoff is from just under $8 \mathrm{kHz}$ to just above $57 \mathrm{kHz}$ at the 5.8 percent model scale. To accomplish this and to allow some control over radial mode content, ultrasonic electrostatic actuators were used. These actuators have a nominal frequency response of $95 \mathrm{~dB}$ SPL at $10 \mathrm{~cm}$ for a $9.9 \mathrm{~V}_{\text {peak-to-peak }} 5 \mathrm{kHz}$ input signal, $\pm 11 \mathrm{~dB}$ from 4 to $110 \mathrm{kHz}$. Dedicated amplifier/power supply assemblies drove the actuators. Figure 2 shows the dimensions of an isolated driver in the standard monopole configuration (Figure 2(a)). The drivers were modified (by removing the 'can' mounted on the backside) so as to provide a dipole source (Figure 2(b)). 

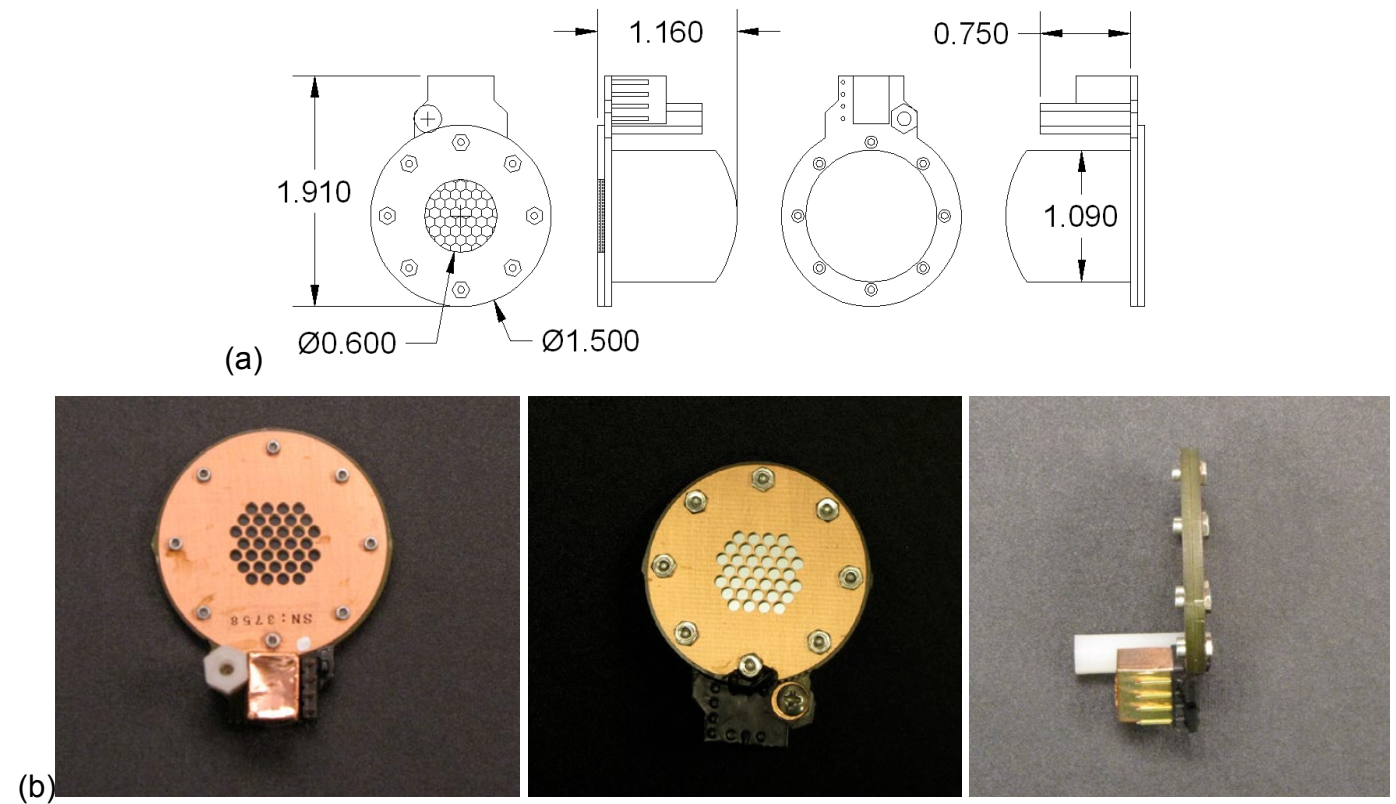

Figure 2.-(a) Dimensions (in inches) of electrostatic actuator in monopole configuration. (b) Photographs of electrostatic actuator in dipole configuration.

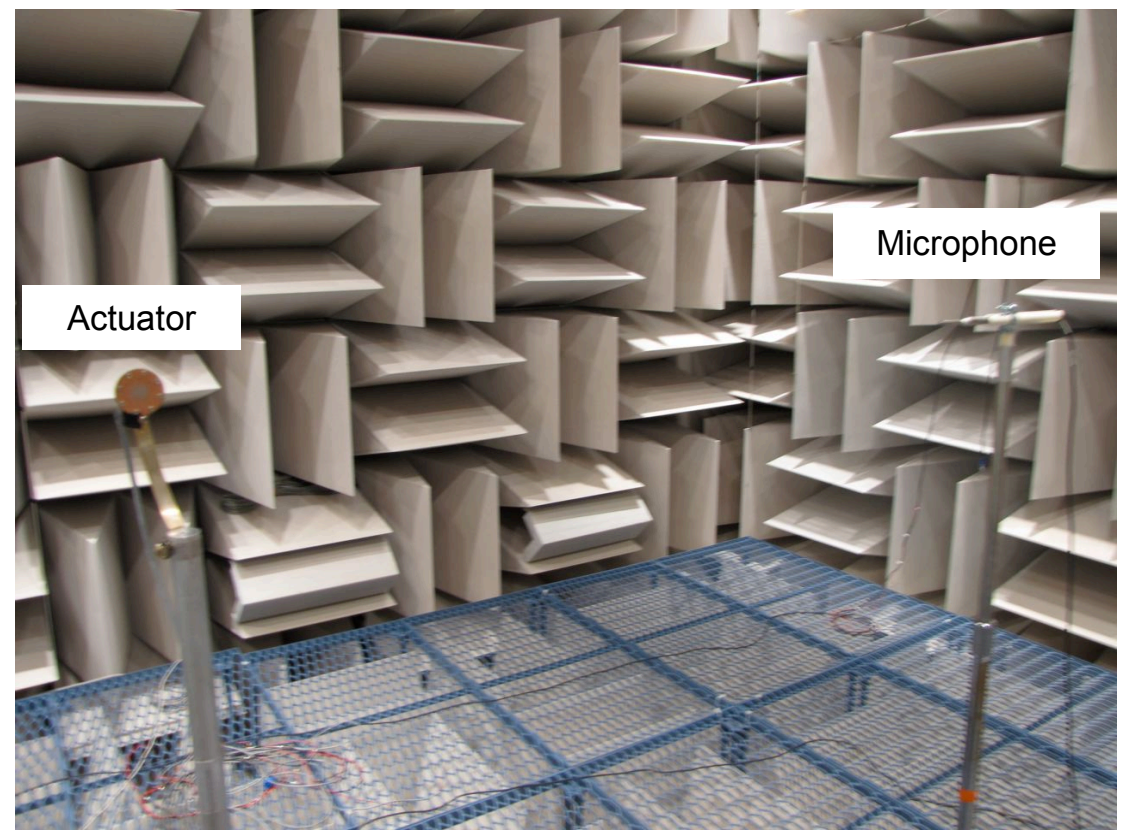

Figure 3.--Isolated actuator test setup in ATL.

The actuators (two standard monopole versions and four dipole configurations) were evaluated individually for free field characteristics by placing them in the ATL (see Figure 3) and measuring the response to a $100 \mathrm{kHz}$ MLS input signal at a $1 \mathrm{~m}$ radius at $15^{\circ}$ increments. MLS (Ref. 9) (or Maximum Length Sequence, sometimes referred to as Gallois Sequence) is a binary pulse-width modulated signal whose circular autocorrelation function is $\boldsymbol{\delta}(0)$. The length of the sequence is $2 \mathrm{~N}-1$, where $\mathrm{N}=18$ was used in this study. At a $100 \mathrm{kHz}$ clock rate, this provides a $2.6 \mathrm{sec}$ burst of deterministic "noise" that contains all frequencies to above $50 \mathrm{kHz}$ but which has a crest factor of 1 , as opposed to Gaussian noise, which has an effective crest factor of over 3. Cross-correlating the excitation signal with a received signal provides the impulse response of the system being measured. A typical frequency response plot is shown in Figure 4(a) and a typical directivity plot in Figure 4(b). The responses were found to be consistent. 


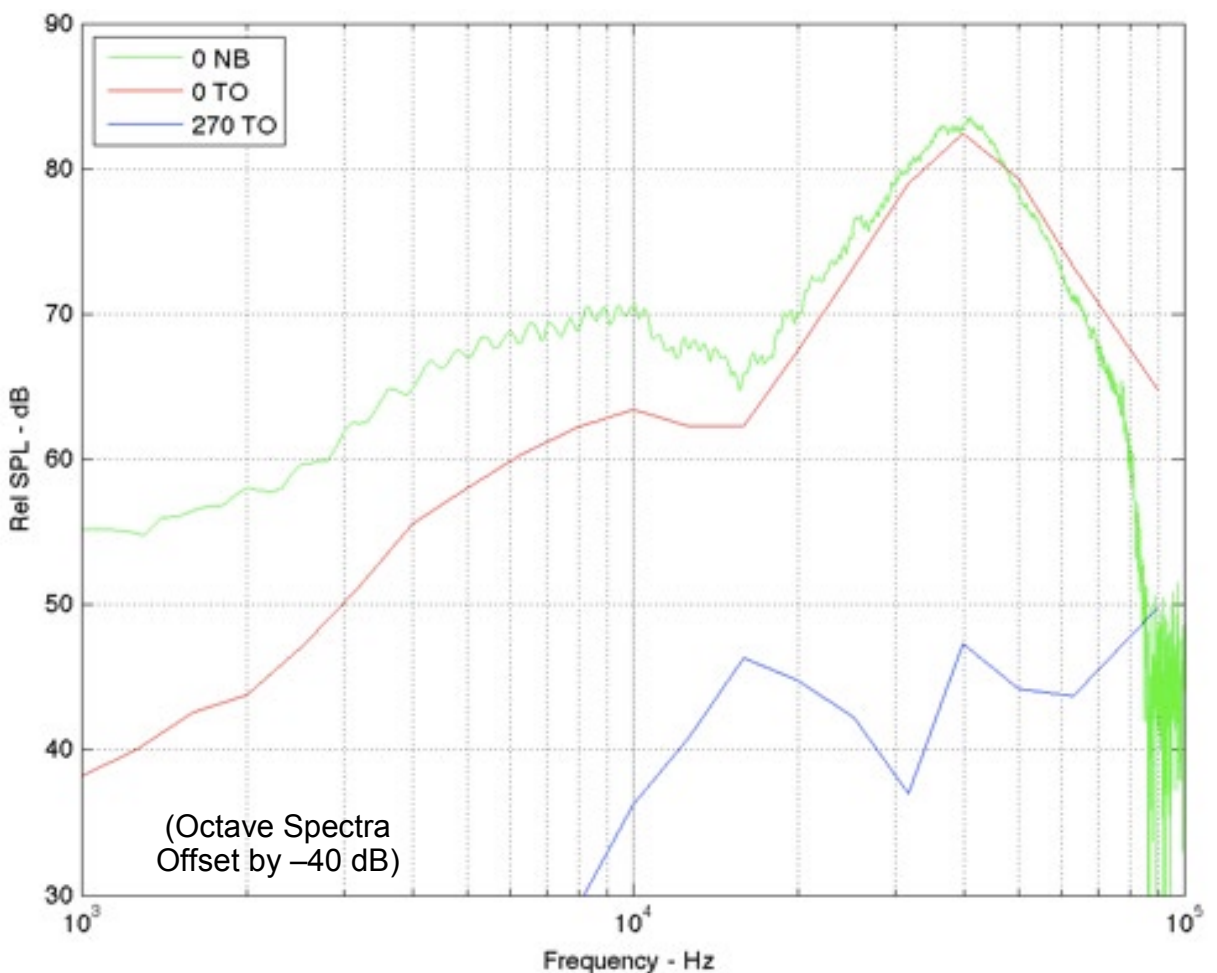

(a)
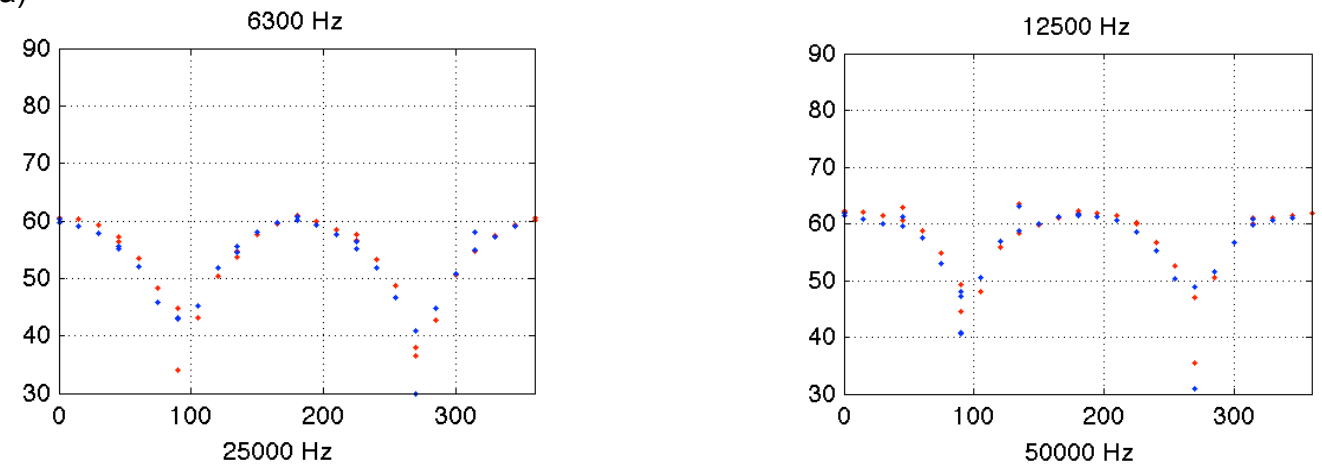

(b)
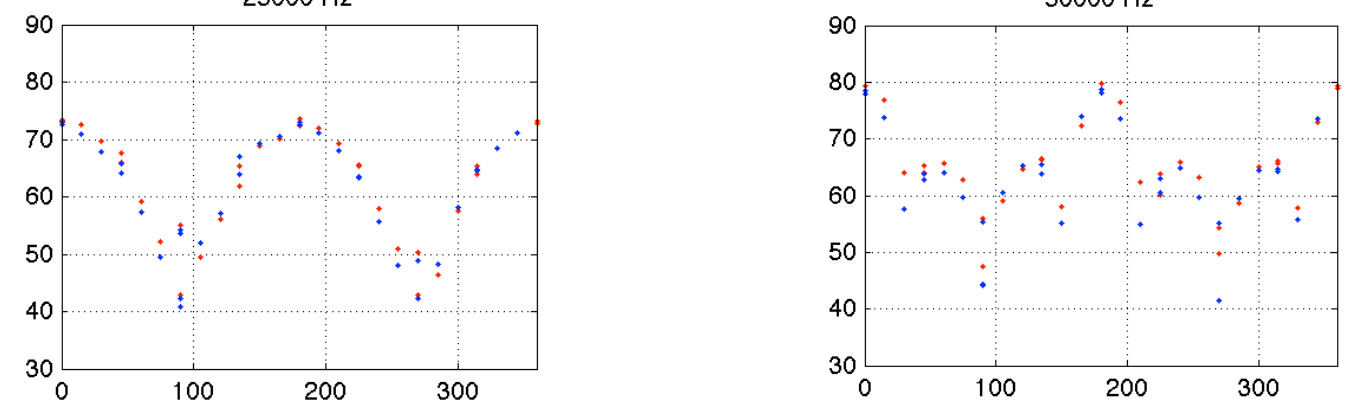

Figure 4.-(a) Typical Narrow Band (NB) and Third Octave (TO) frequency response of isolated actuator (dipole configuration) at $0^{\circ}$ and $270^{\circ}$ from principal radiation axis. (b) Typical directivity response (decibel versus angle) of isolated actuator (dipole configuration).

Each actuator and in-duct microphone was bench-tested for complex frequency response and equalization tables created to minimize the effect of actuator nonuniformity on modal radiation and of microphone nonuniformity on modal analysis. Actuators were driven from a dedicated D/A system using prerecorded 37-channel multifrequency signals (one for each actuator and one reference) WAV file. Induct microphone data were recorded on a separate dedicated A/D system, resulting in a 73-channel (one for each microphones and reference) TDMS file (a National Instruments (NI) file format). Far-field data 
was recorded on a dedicated facility A/D system, resulting in a 16-channel file (one fixed microphone, 13 traversing microphones, one reference and one timing signal). For each position of the far-field microphone traverse, three combinations of tone excitation and a 3-sec burst of 36-channel statistically independent random noise were recorded to provide differing radial mode mixes and to compare data being taken in a separate test using pneumatic broadband noise generation.

\subsection{Internal Microphones}

The acoustic signature was measured in-duct, for modal content verification, by omnidirectional electric condenser microphones (Figure 5). These microphones are typically used in the audible range (20 to $16,000 \mathrm{~Hz}$ ) but have been utilized for ultrasonic wildlife studies (Ref. 10). These microphones were also evaluated for frequency response and compared to the response of a $1 / 4 \mathrm{in}$. B\&K 4939 style microphone (Figure 6). Microphones whose response was relatively inconsistent were not used.

Modal separation analysis of the $3 \times 24$ in-duct microphone rings showed considerably more apparent scattering and aliasing than had been expected, particularly for the HWB mode set. A potential cause for this would be nonlinearity in microphone amplitude response. For example, with BPF, 2BPF and 3BPF signals driving the actuators at approach conditions, nonlinear microphone response would generate a fictitious series of harmonics, sum and difference signals that could significantly distort the phase response at individual microphones or entire microphone rings, leading to false circumferential and radial mode identification.

Dimensions in $\mathrm{mm}$ (not to scale)

WM-64C/64K

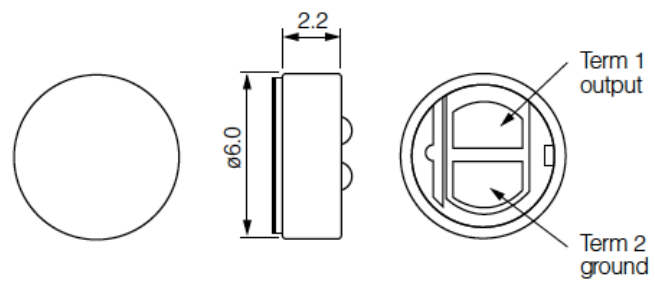

Figure 5.-Dimensions of Electret Microphone.

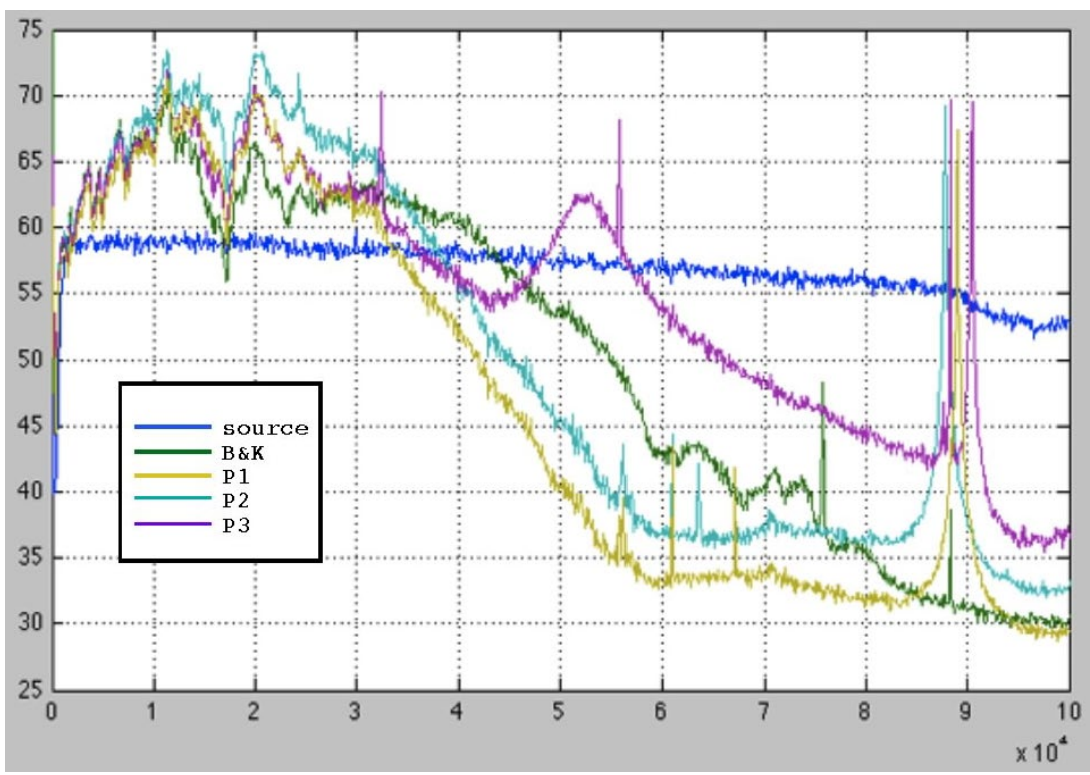

Figure 6.-Sample isolated electret microphone frequency response curves compared to $1 / 4$ in. B\&K 4939 style microphone. 
A review of the interface circuitry between the in-duct microphones and the National Instruments (NI) signal conditioner/data acquisition system revealed that in order to accommodate the fixed 4-mA bias current provided by the NI system, the microphones were connected with $620 \mathrm{ohm}$ shunt resistance, which forms the effective load on the microphones' built-in FET preamplifier. Since the rated minimum load impedance is 2,200 ohms, a possible source of nonlinearity was identified (see Figure 7).

A substitution test was conducted to compare a spare in-duct microphone as configured in the test to a laboratory $1 / 4 \mathrm{in}$. condenser microphone. The test instrument was restricted to $20 \mathrm{kHz}$ but the results are well demonstrated. Several tone combinations were applied to a high frequency loudspeaker and harmonic and intermodulation distortion observed. The CVM and HWB mode signals (up to $20 \mathrm{kHz}$ ) are shown in Figure 8 . The lab microphone levels are offset $-20 \mathrm{~dB}$ for purposes of illustration.

The CVM frequencies are spaced every $1 \mathrm{kHz}$ with a gap at $10 \mathrm{kHz}$. The harmonic and intermodulation distortion products for the lab microphone (blue) are 40 to $60 \mathrm{~dB}$ below the excitation tones and are most likely generated by the test loudspeaker rather than the microphone. However, the distortion products for the example in-duct microphone are only about $20 \mathrm{~dB}$ below the excitation tones. For purposes of the UCFANS test, 7 and $14 \mathrm{kHz}$ are the only target signals that correspond to primary harmonic or IM interactions, so the nonlinearity is not expected to have a strong effect on the CVM analysis results.

Only four of the seven HWB mode frequencies fall within the $20 \mathrm{kHz}$ analysis limitations. However, the relatively strong difference tone at $12 \mathrm{kHz}$ illustrates that the interactions are present. Detailed computation of the influence of nonlinearity on the mode analysis results has not been attempted. However, for future use, the potential for contamination could be reduced significantly by maintaining the minimum load impedance or greater. As an example, the difference between $620 \mathrm{ohms} \mathrm{load} \mathrm{and} \mathrm{the}$ minimum 2,200 ohm load is shown in Figure 9. Using the minimum recommended load impedance does not eliminate the distortion products, but reduces them significantly. It is expected that dedicated preamplifiers with optimized load impedance would reduce the distortion by at least $10 \mathrm{~dB}$ compared to the $620 \mathrm{ohm}$ configuration.

In order to better assess the influence of this nonlinearity on actual UCFANS data, full spectra (up to $40 \mathrm{kHz}$ ) were computed for the microphones on a representative CVM and HWB measurements. Results are shown in Figure 10(a) and (b). It can be seen that whereas the distortion products in the bench test were in the range of $-20 \mathrm{~dB}$ (10 percent), the in-duct amplitudes were lower than that used in the bench test and are in the range of -40 to $-30 \mathrm{~dB}$ ( 1 to 3 percent); thus the distortion is unlikely to have a significant influence on the mode separation. An exception could be the approach BPF signal, where the frequency of the second harmonic $(14,571 \mathrm{~Hz})$ is very close to the frequency of the first harmonic at cutback $(14,247 \mathrm{~Hz})$. For future use it is recommended that a more optimized load be used for the in-duct microphones.
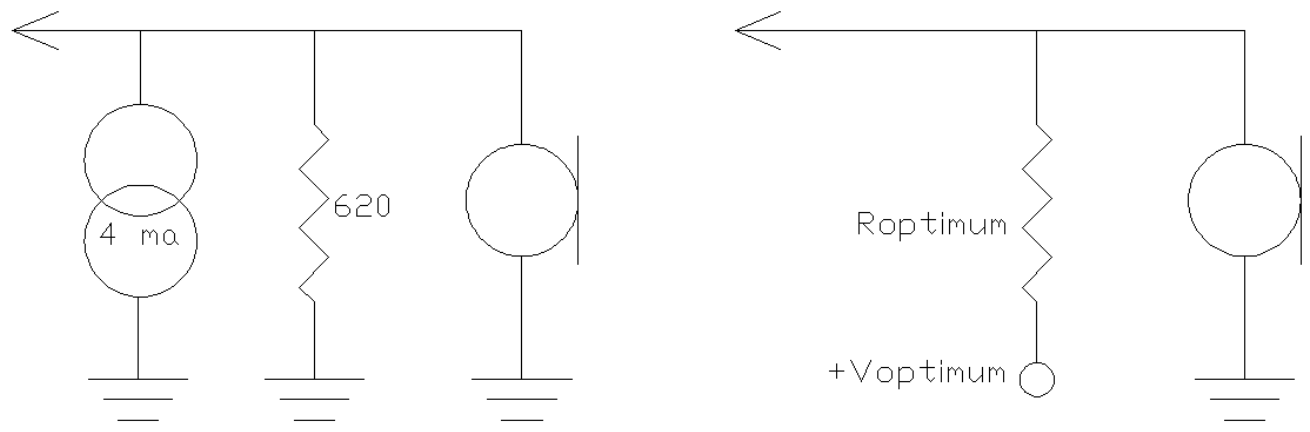

Figure 7.-As installed (left) and preferred microphone connections. 
Mic 107 to LD 1/4" Compare CVM

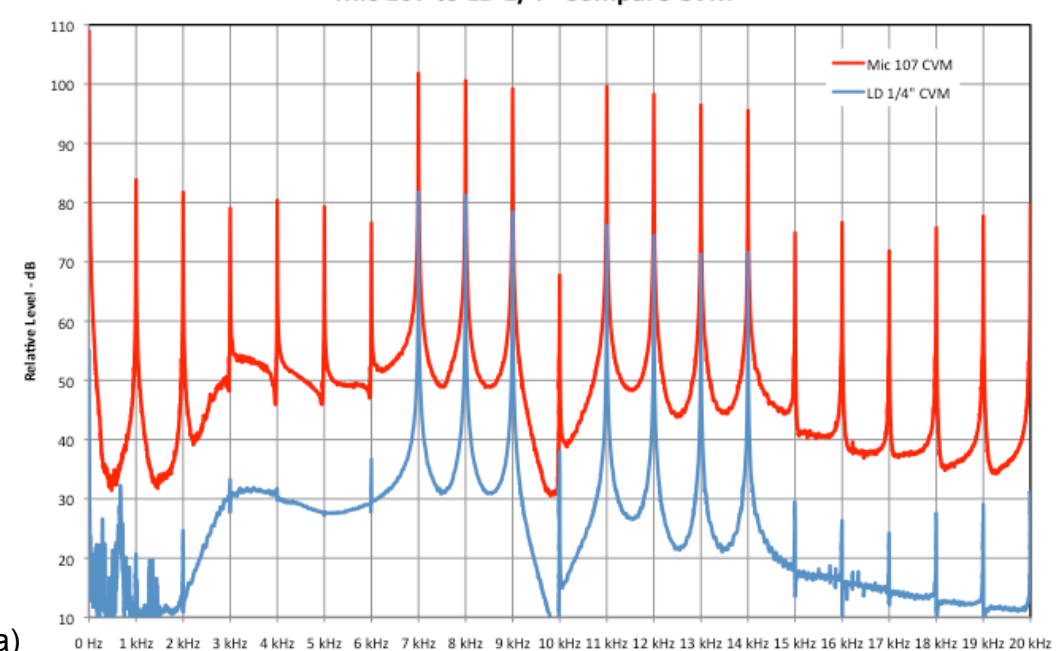

(a)

Mic 107 to LD 1/4" Compare HWB

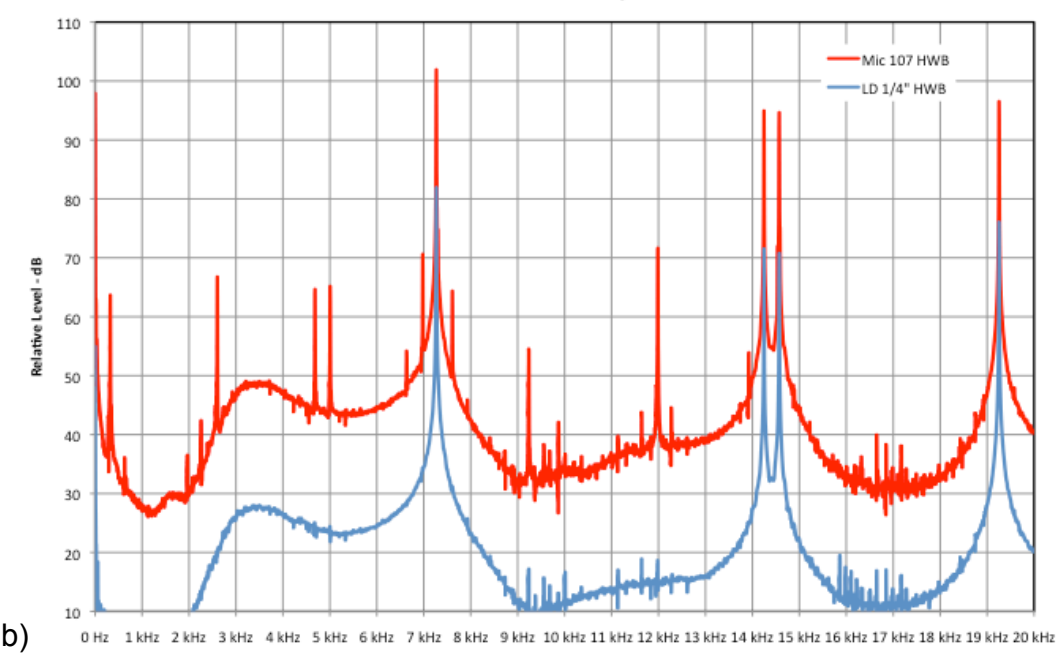

Figure 8.-Comparison of distortion products for laboratory and UCFANS in-duct microphones. (a) CVM and (b) HWB.

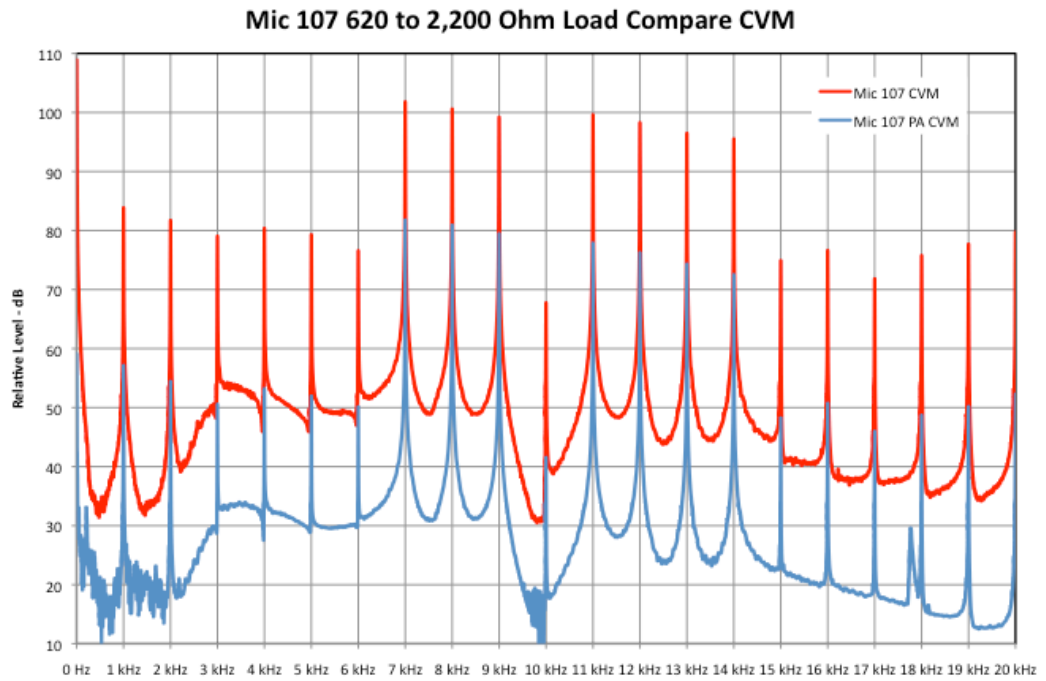

Figure 9.-Demonstration of distortion reduction with increase of UCFANS induct mic load impedance. 


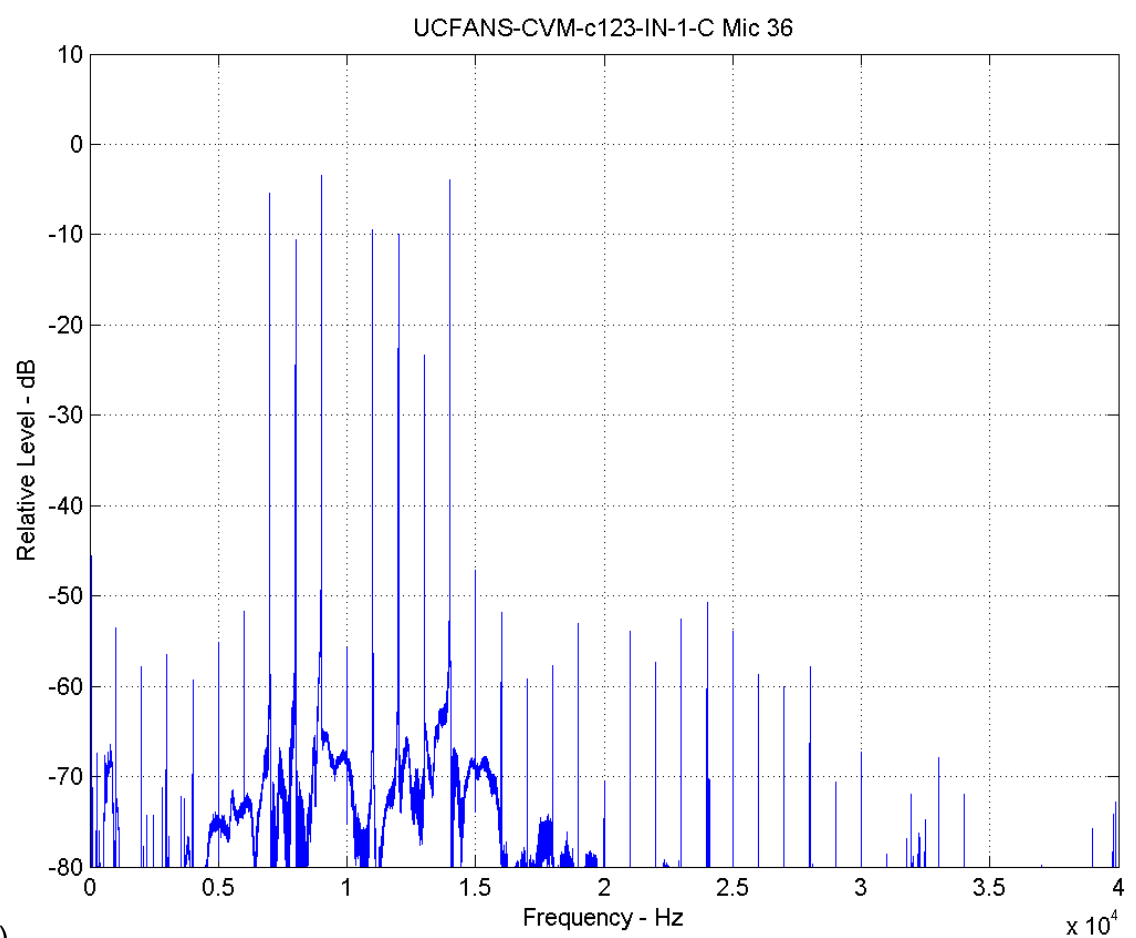

(a)

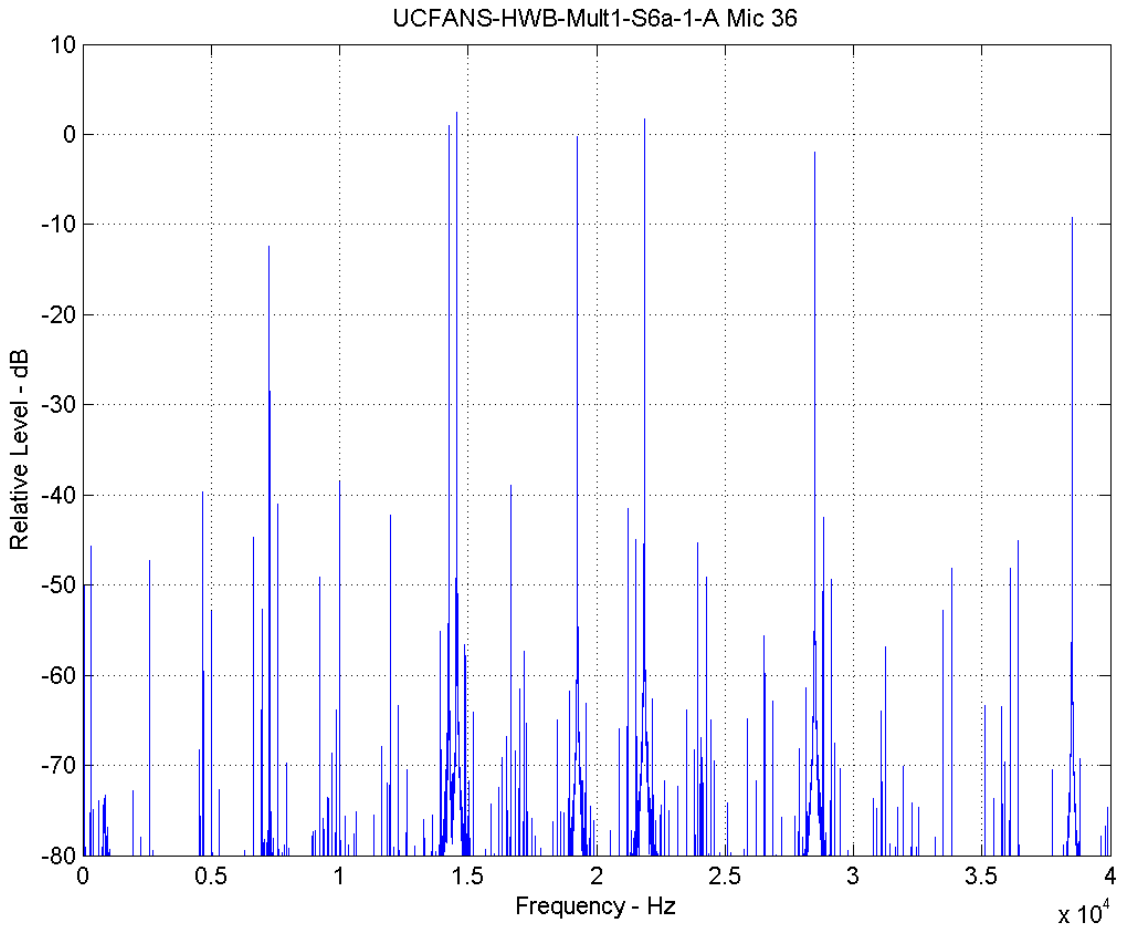

Figure 10.-(a) Representative distortion components from actual UCFANS measurement-HWB. (b) Representative distortion components from actual UCFANS measurement-CVM.

\subsection{Assembly}

Drawings of the model are shown in Figure 11. Figure 11(a) is a line drawing with critical dimensions. Figure 11(b) is the CAD drawing showing the components. Areas critical to far-field acoustic radiation from the duct, such as inlet lip and duct exit dimensions were held to high fidelity, while areas 
not so critical to acoustics (e.g., internal flow path) were relaxed. The rapid prototype model was cast in five sections so that any section could be removed, replaced, or redesigned. In particular the exhaust lip and tail cone can be removed and replaced with the inlet lip and spinner to switch the model from an exhaust to an inlet radiation model. In these cases, the opposite end of the model is blocked off, and absorptive material placed in the cavity to minimize internal reflections. The remainder of the model is unchanged. The cabling for the internal drivers was routed through the center section. While this arrangement meant that the complete internal and external lines did not completely match the target nacelle, this area is not primarily relevant to duct/far-field radiation, and furthermore the prime objective of the test was to obtain the differences between the shielded and unshielded cases. The flexibility in this arrangement (i.e., no rewiring actuators or moving the model required to convert between the exhaust and inlet configurations), more than outweighed any minor effect on the radiation. An appropriately scaled pylon was placed between the nacelle and wing to simulate the mounted geometry on the HWB.

(a)

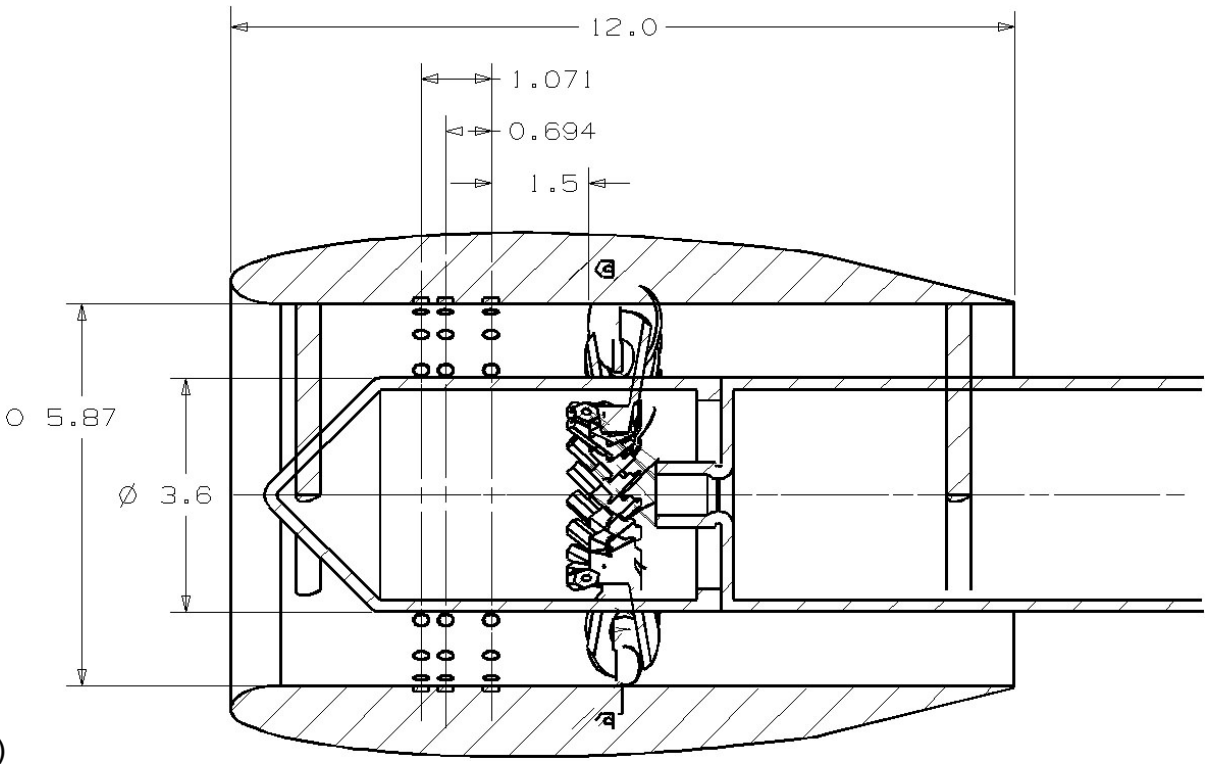

(b)

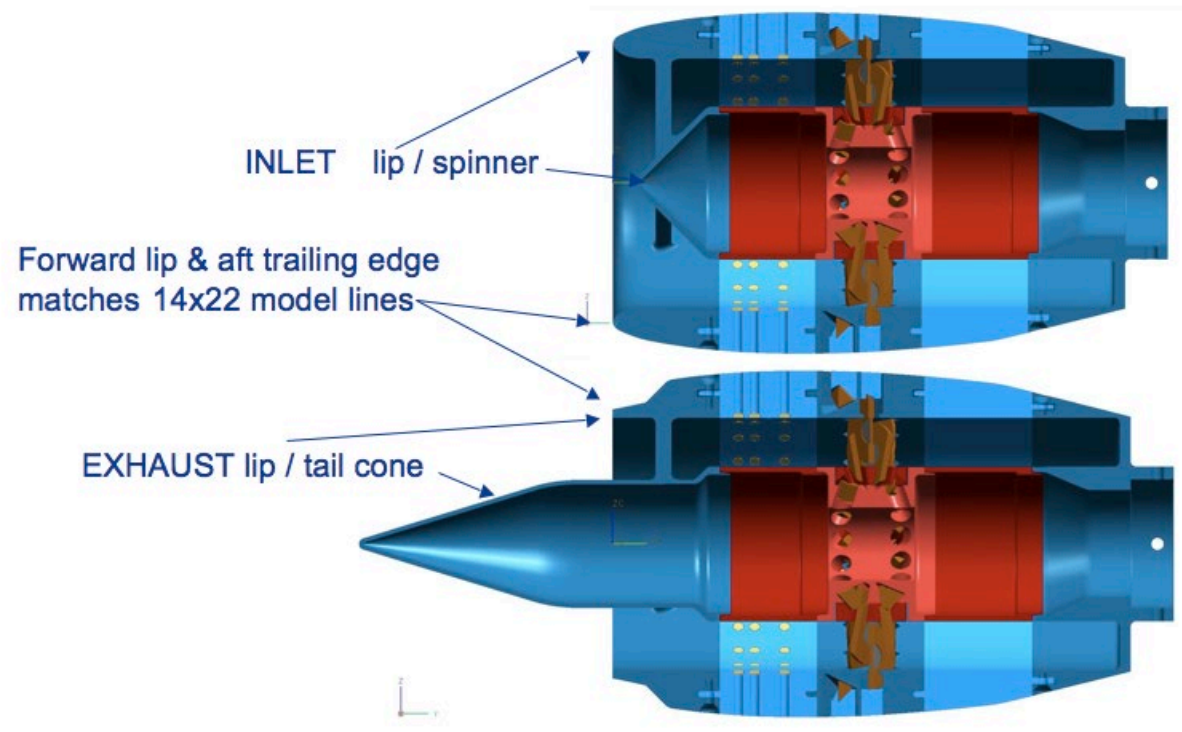

Figure 11.-(a) Line drawings of nacelle in inlet configuration. (b) CAD drawings of nacelle in inlet and exhaust configurations. 
Figure 12 shows photographs of the actuators installed. The actuators were mounted in an annular ring, whose dimensions matched the hub-to-tip ratio of the proposed N2A engine nacelle. The inner path was kept constant to the spinner to minimize mode change due to area. Two rows of 18 actuators each were mounted circumferentially, one from the hub, and the second row from the 'tip'. These rows were offset radially in the same axial plane, but interlocking. The actuator count and distribution allowed for circumferential modes up to $m=8$, and 2 radial modes to be controlled. Higher modes can be generated but effects like aliasing and under specification become factors. The microphones were flush mounted internally in three axially distributed rows, 24 microphones each, equally spaced in the circumferential direction (Figure 13). This distribution allows for measurement of up to $m=11$, and $n=2$, without aliasing. Compensation curves for all microphones were obtained in-situ.
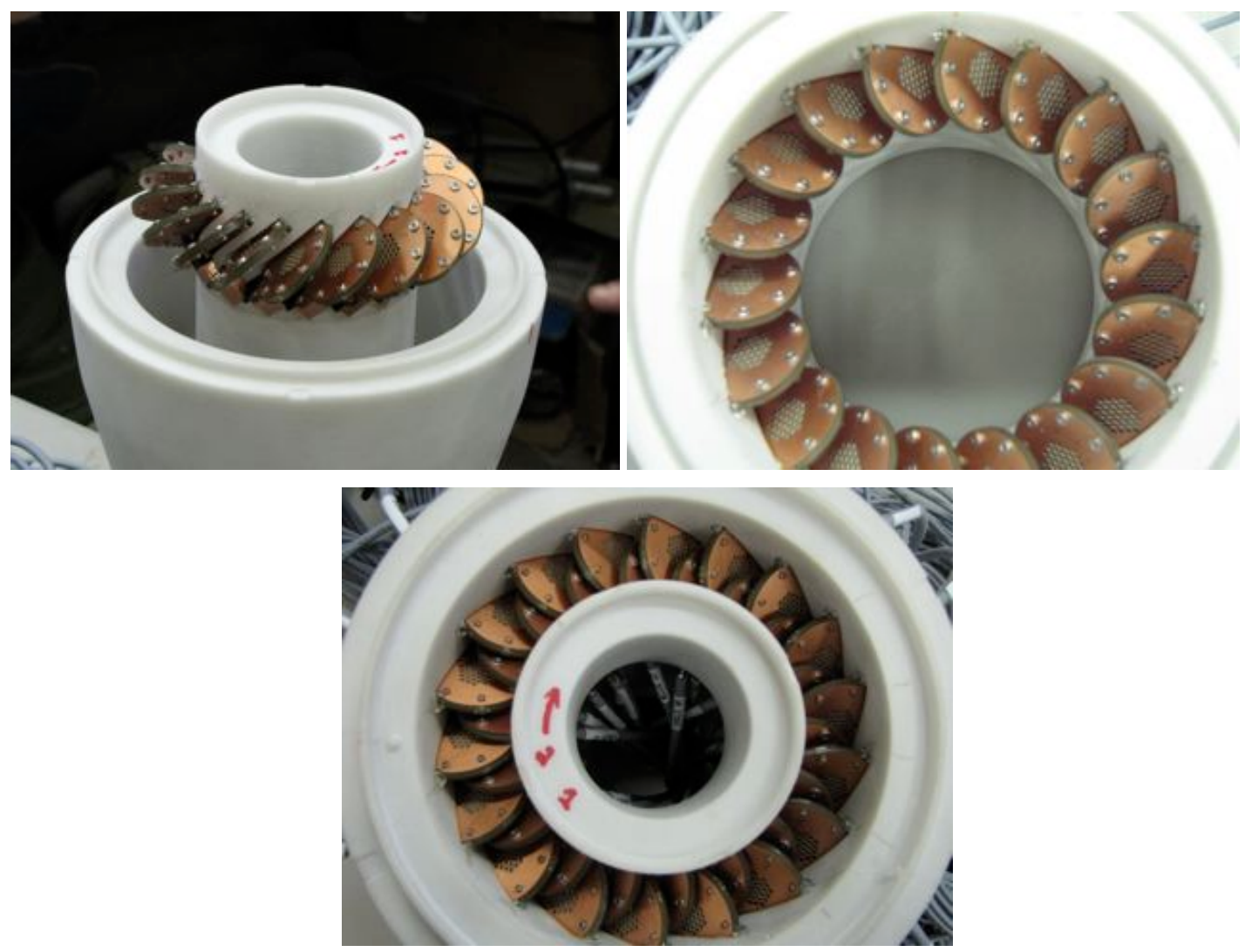

Figure 12.-Actuators installed in rapid prototype model.

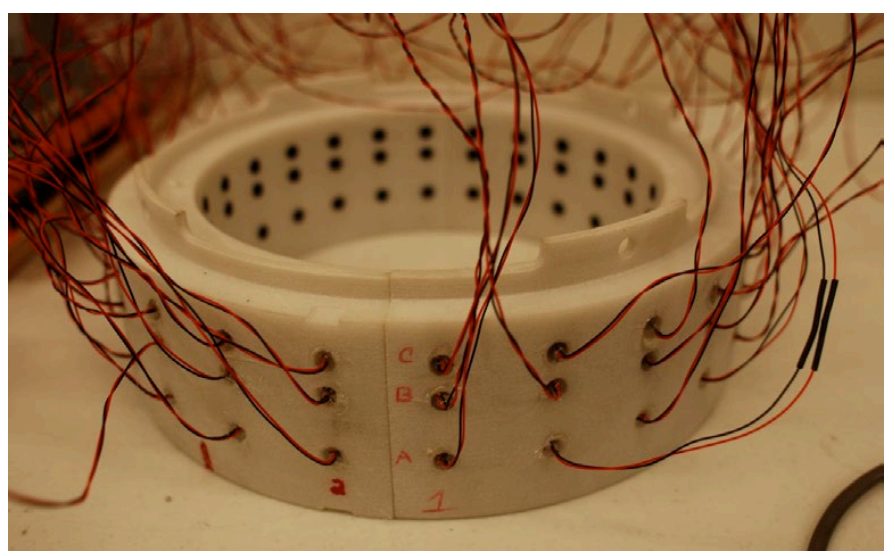

Figure 13.-Electret microphones installed in rapid prototype model. 
Figure 14(a) shows the fully assembled (undressed) UCFANS nacelle and Figure 14(b) shows the nacelle mounted in the ATL.

(a)
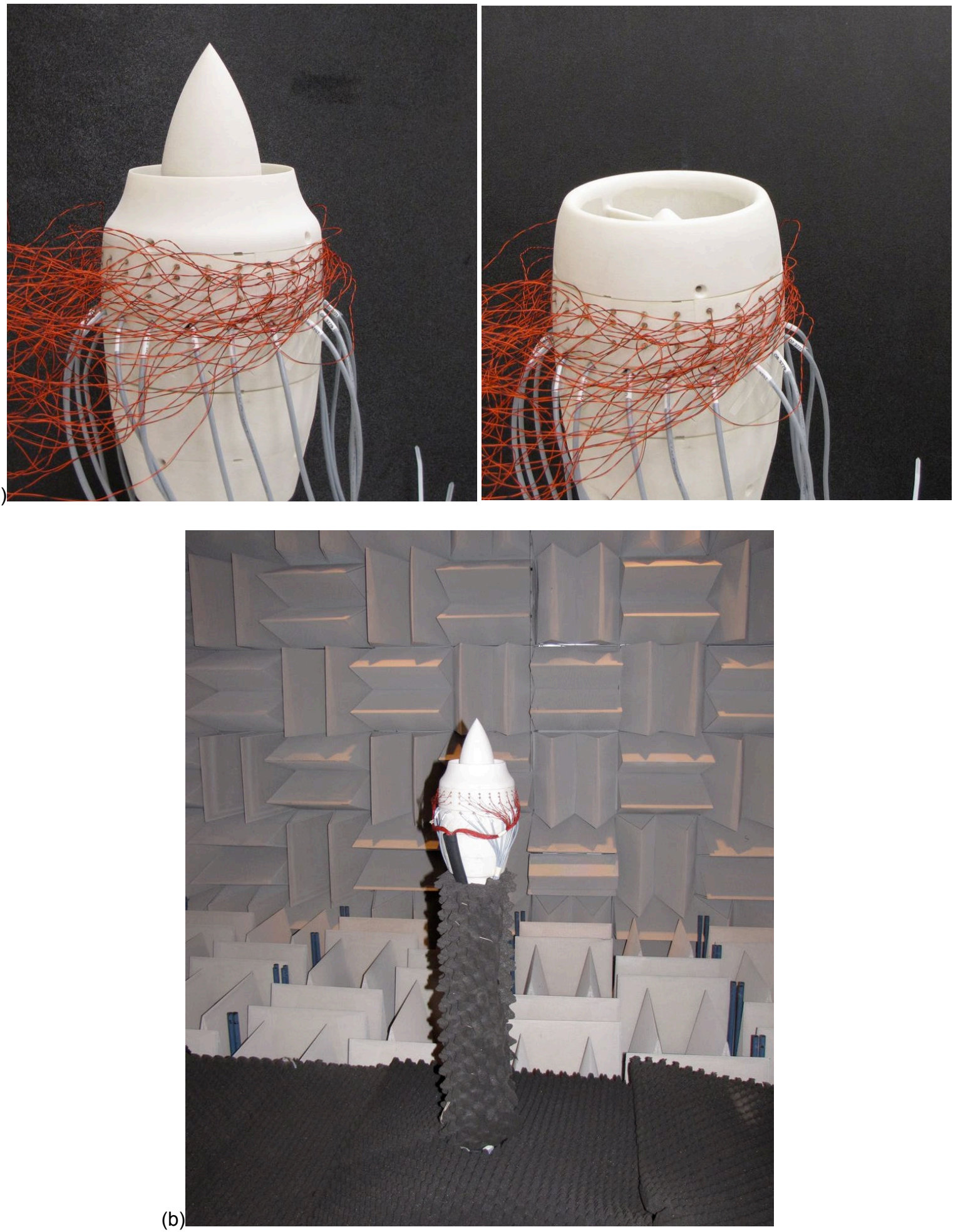

Figure 14.-(a) Assembled rapid prototype model in exhaust, and inlet configurations (not dressed). (b) UCFANS in exhaust configurations installed in ATL. 


\subsection{Shield Hardware}

The wing, or shield, was an aluminum plate $68.5 \mathrm{in}$. high that spanned the entire width of the acoustic chamber. It was decided to trade-off the 3-D plan form effects present on the HWB to concentrate on the diffraction effects of the edge. For the UCFANS in the exhaust configuration, a sharp trailing edge based on the HWB N2A-EXTE model was used. The trailing edge was made of solid rapid prototype material and was mounted on, and blended to, the aluminum plate (see Figure 15(a)). The aft-most portion of the trailing edge part matched the N2A model lines then was blended back to the $1 / 4$ in. plate. For the UCFANS in the inlet condition, the edge is represented by more of the hybrid wing fuselage and was made of fiberglass. The trailing edge is affixed to the wing/wall as seen in Figure 16. The leading edge was made of fiberglass and fits over the trailing edge so that the edges may be reused as illustrated in Figure 15(b). It, too, matches the N2A lines to a point, then is faired back to blend into the plate. This arrangement captures the primary effect due to acoustic scattering at the edges and plan-form shielding while maintaining model simplicity.

Trailing edge rapid prototype

(a)

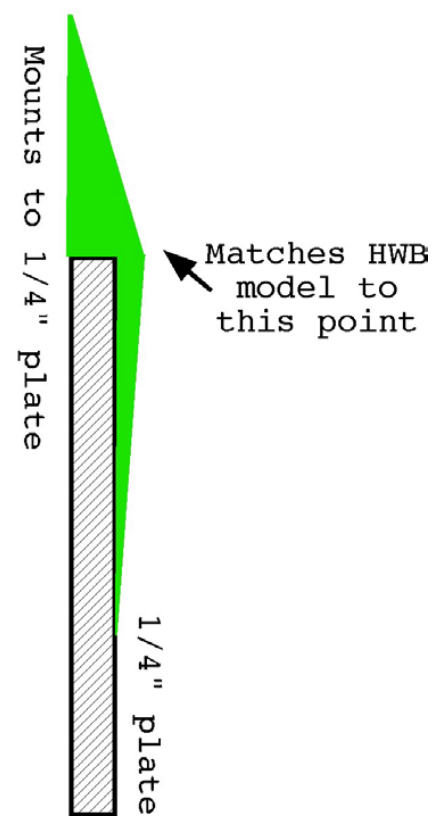

(b)

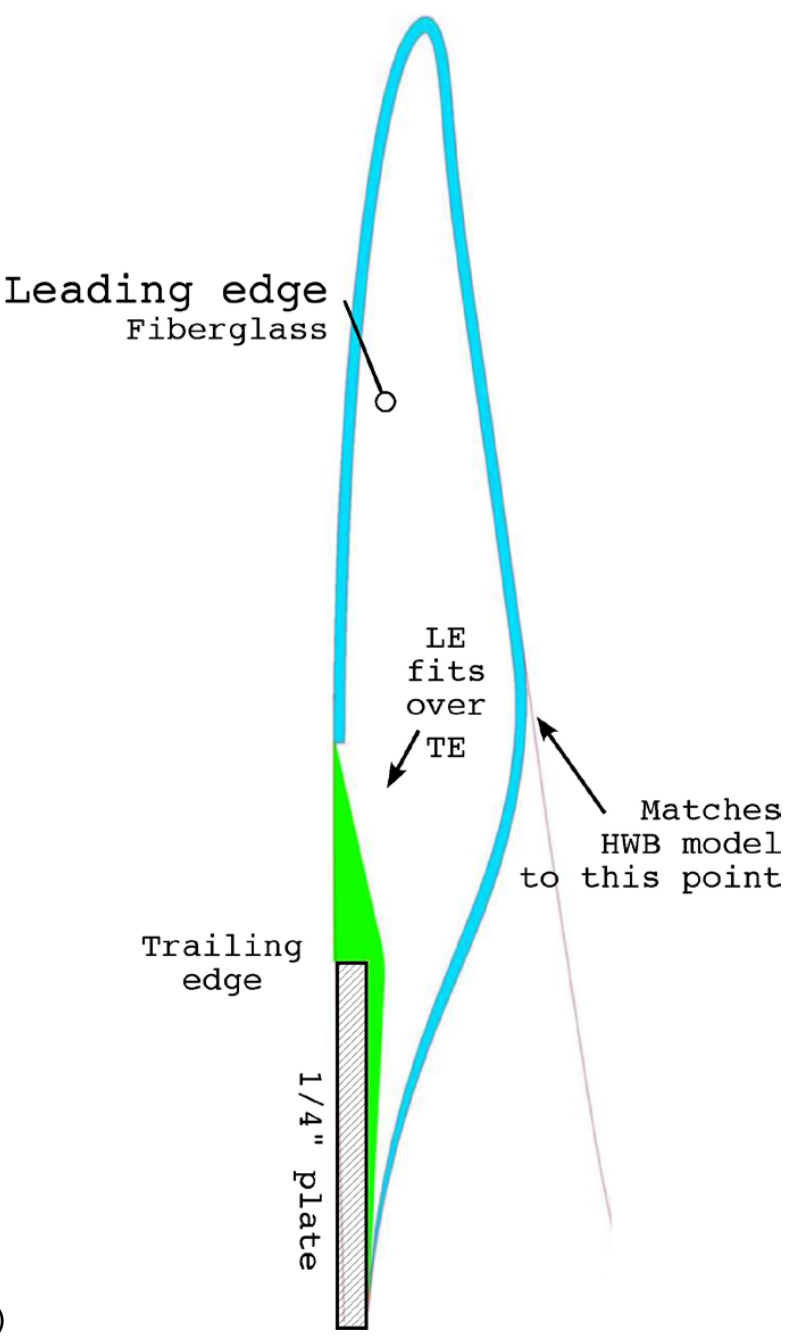

Figure 15.-(a) Trailing edge representation, and (b) leading edge representation. 


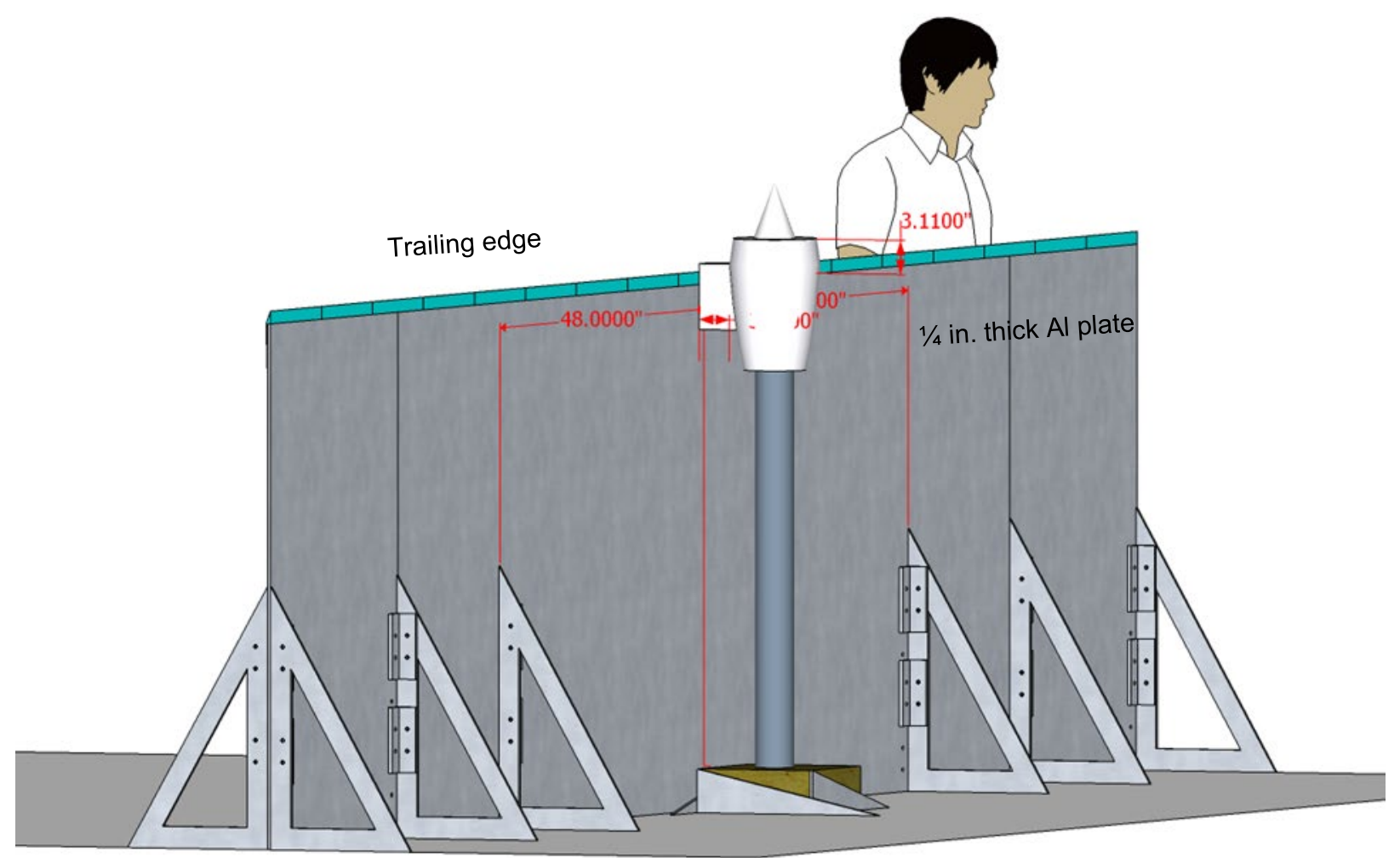

Figure 16.-Trailing edge affixed to wing/wall.

Two pair of verticals based on the N2A model verticals were manufactured using rapid prototyping. Two sets were built, one with a $10^{\circ}$ cant, and one with a $30^{\circ}$ cant. Each pair was tested at two different axial positions relative to the trailing edge. The two positions as measured from the trailing edge of the wing to the trailing edge at the base of the vertical were $x_{\text {vert }} / d=0.90$ and 1.41.

These were mounted in pairs on the shield wall to determine their effect, if any, on the shielding values.

Figure 17(a) and (b) show the positions of the verticals relative to the nacelle and the trailing edge (note: the two cants are superimposed in the drawing but were tested separately). The pair of verticals mounted in each configuration were spaced apart from one another in the properly scaled distance, and the single nacelle was positioned at the starboard engine location of the N2A.

\subsection{In-Duct Signature Generation and Validation}

A block diagram of the signature generation and in-duct measurement is presented in Figure 18. 


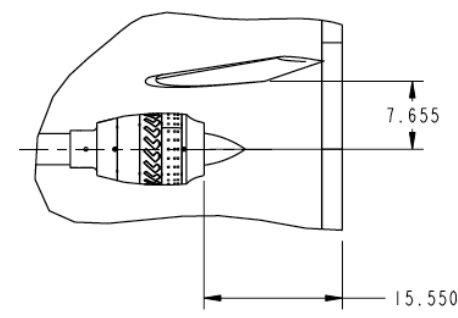

$10^{\circ}$ DEGREE FORWARD TOP VIEW

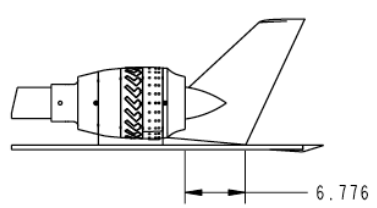

$10^{\circ}$ DEGREE FORWARD SIDE VIEW

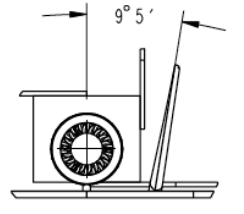

$10^{\circ}$ DEGREE FORWARD BACK VIEW

(a) Verticals at $x_{\text {vert }} / d=0.4, \delta=10^{\circ}$

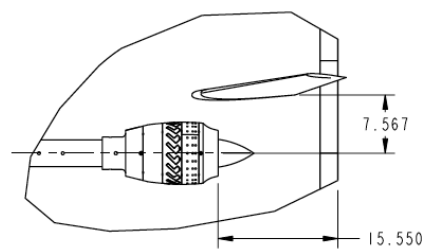

10 DEgREE AFT TOP VIEW

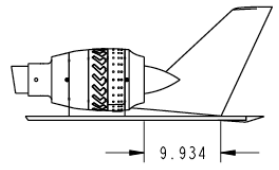

I0 DEGREE AFT SIDE VIEW

(c) Verticals at $x_{\text {vert }} / d=0.9, \delta=10^{\circ}$

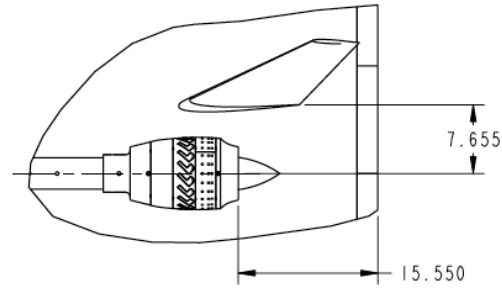

$30^{\circ}$ DEGREE FORWARD TOP VIEW
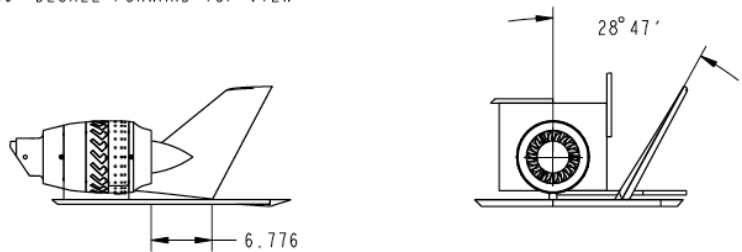

$30^{\circ}$ DEGREE FORWARD SIDE VIEW

$30^{\circ}$ DEGREE FORWARD BACK VIEW

(b) Verticals at $x_{\text {vert }} / d=0.4, \delta=30^{\circ}$

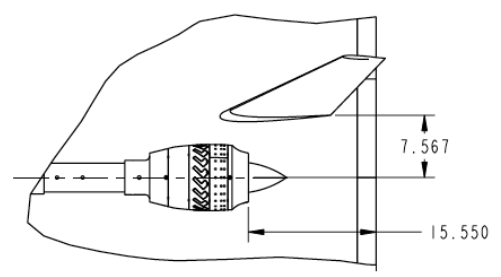

$30^{\circ}$ DEgREE AFT TOP VIEW
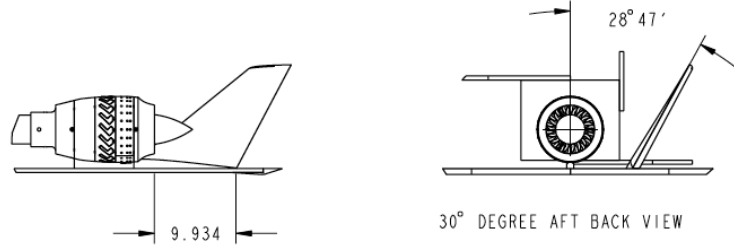

$30^{\circ}$ DEGREE AFT BACK VIEW

$30^{\circ}$ DEGREE AFT SIDE VIEW

(d) Verticals at $x_{\text {vert }} / d=0.9, \delta=30^{\circ}$

Figure 17.-Sketches of vertical in two positions at $10^{\circ}$ and $30^{\circ}$ cants. 


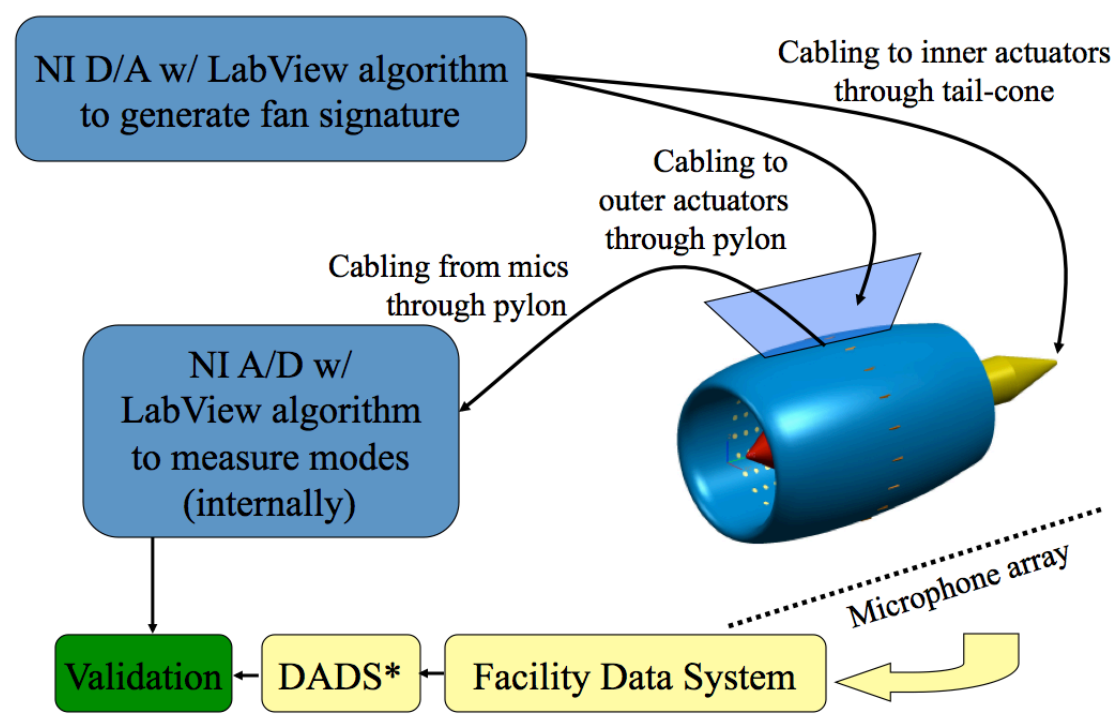

Figure 18.-Block diagram for UCFANS signature generation and measurement ( ${ }^{*}$ Digital Acoustic Data System).

\subsubsection{Signature Generation}

The signature to generate the modal content was precalculated using the desired modes and frequencies, and stored. Each actuator in the array was driven by a composite signal of seven excitation frequencies, equalized for variations in individual actuator amplitude and phase response, and phased to match the circumferential wave numbers of the modes to be radiated. A GUI program was used to create the algorithm to read in the prestored signal. These 36 signatures, plus a reference signal were output by a $200 \mathrm{kS} / \mathrm{s}$ sample rate D/A chassis, allowing the full desired frequency range to be well below the Nyquist frequency. In addition to the 36-actuator drive channels, a reference channel consisting of the unity amplitude cosine wave of all excitation frequencies that was output directly to the data recorder. Because the test frequencies were limited to integer values up to $60 \mathrm{kHz}$, the excitation signals were multiples of $1-\mathrm{sec}$ duration.

An additional matrix of 36 each, $3 \mathrm{sec}(600,000$ points) Gaussian noise signals was generated and stored in a file and applied to the test fixture actuators as an additional file. Care was taken to ensure that although the signals are statistically independent among the actuators, the radiated signal is coherent from test point to test point, allowing cross correlation and coherence computation between data taken at different traverse stops or even on different test days.

To minimize test time, three unique blocks of the seven frequencies plus the broadband signature (Gaussian distribution) were generated, sequentially. The two sets of radial drivers could be preset with a desired amplitude and/or phase relation, as a group. To achieve differing radial mode combinations, three consecutive, 2-sec signal bursts were applied with differing drive levels to the inner and outer actuators rows. Since the signal is known, parsing the frequency content from each block separately was conceptually straightforward. Effectively, this allowed for 22 separate conditions to be acquired in a short time frame. Figure 19(a) depicts the sequential block concept and Figure 19(b) the FFT of a representative block. 


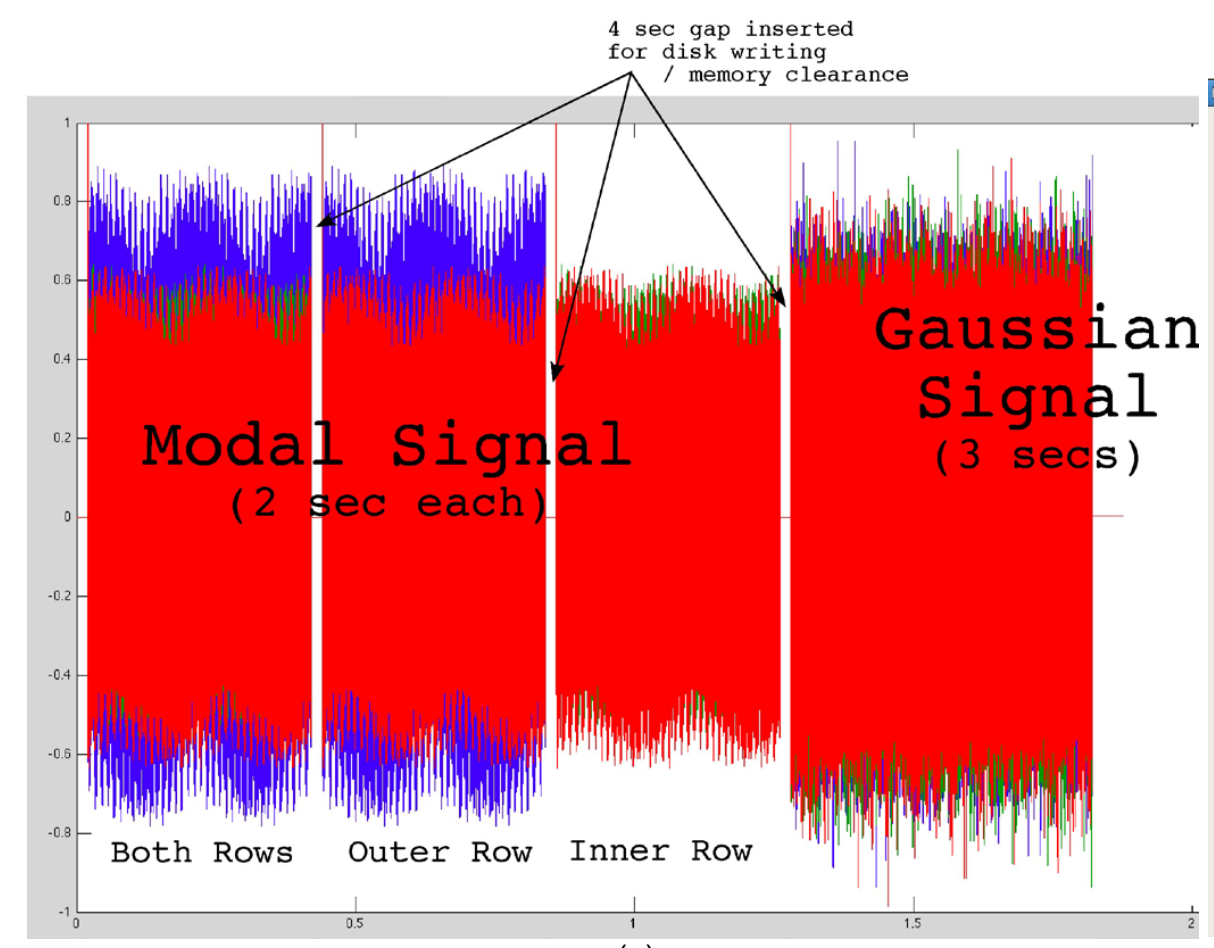

(a)

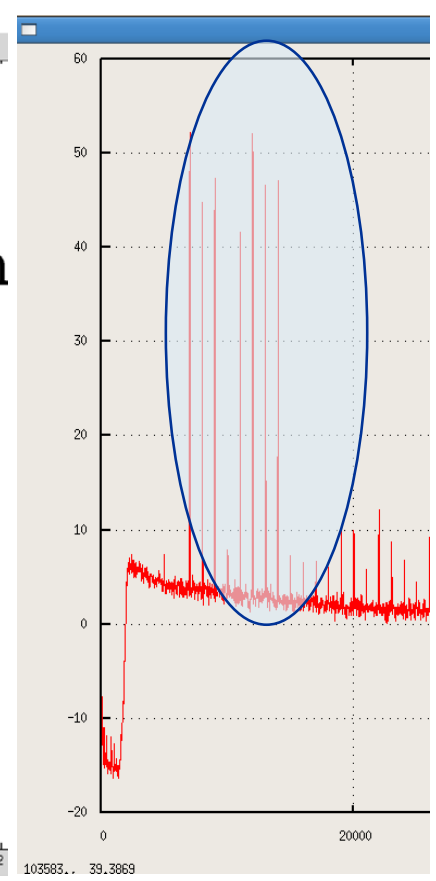

(b)

Figure 19._UCFANS signature. (a) Illustrative time history, and (b) spectral content.

\subsubsection{Signature Validation}

The mode-identification microphone array consists of 72 prepolarized condenser microphones in three rows of 24 each, allowing resolution of circumferential modes up to $|m|=11$. The array is located approximately midway between the actuators and the duct termination, at axial positions that were determined to allow identification of radial orders $n=0,1$ and 2 over the BPF, and part of the $2 \mathrm{BPF}$ frequency range 7 to $30 \mathrm{kHz}$.

The 72 internal microphones, along with the reference signal, were acquired using an AC-coupled $200 \mathrm{kS} / \mathrm{s} \mathrm{A} / \mathrm{D}$ converter with integrated anti-aliasing filters. These time histories were streamed to disk for later analysis. The 72-microphone signals and a reference signal were recorded at $200 \mathrm{kHz}$ sampling rate using simultaneous sampling. The cross-power spectrum of each microphone channel was computed against the reference signal and spectral components corresponding to excitation frequencies were isolated into a "compressed spectrum." The compressed spectrum for each microphone was equalized based on that microphone's calibration curve and then the 24 spectra from each microphone ring were spatially Fourier transformed to recover the complex amplitude of each circumferential mode order $-12<m<12$, with $m=|12|$ measurable, but the direction indeterminate. 
For each circumferential mode $m$ of frequency $f$, the radial component composition was estimated by computing the modal cutoff ratios and axial wave numbers

$$
k_{z_{m, n}}=\frac{2 \pi f}{c} \sqrt{1-\left(\frac{f c o_{m, n}}{f}\right)^{2}}
$$

for all radial orders $n=0$ to one above the highest cut on.

For microphone row $\mathrm{A}, \mathrm{B}$, or $\mathrm{C}$, an axial coordinate $\mathrm{z}$ was established relative to the midway point between rows $\mathrm{A}$ and $\mathrm{C}$. The transfer function from this location to each microphone row $M$ and each propagation mode $(m, n)$ is then

$$
p_{M, m}=\sum_{n} p_{0, m, n} \exp \left(-i k_{z_{m, n}} z_{M}\right)
$$

or, expressed as a transfer matrix function

$$
\left[p_{M}\right]_{m}=\left[H_{M, n}\right]_{m}\left[p_{0, n}\right]_{m}
$$

Since complex circumferential mode amplitudes $p_{M}$ are determined from spatial Fourier transform of the microphone data, the matrix $H$ is inverted and multiplied with $p_{M}$ to determine the radial content, $p_{0, n}$ relative to the center of the microphone array. This inversion was done using MATLAB PINV function, which has the properties:

If rows $=$ columns, inversion is exact

If rows $>$ columns, inversion is the minimum norm solution (fewer modes than microphone rows)

If rows $<$ columns, inversion is least squares error solution (more modes than microphone rows)

Because each test involved hundreds of far-field microphone array traverse stops, with the same excitation signals applied to the simulator drivers, the modal composition of the excitation was computed for a representative subset of the traverse stops. The results were compared for consistency to ensure repeatability of the radiated sound field.

Two methods were employed to separate the duct propagation modes from the 72 microphone signals: a) A two-step process that separates circumferential modes in each microphone ring and then separates radial modes by inversion of a transfer matrix based on modal axial wavenumber; b) A simplified "beamforming" approach that uses the modal transfer functions to each microphone as steering vectors and the cross-spectral matrix of the 72 microphone signals. For the two-step process, only unaliased circumferential modes $(-12<m<=12)$ were considered. In the beam-forming process, the first cycle of aliased modes was also considered $(-24<m<=24)$. In either case, only radial orders $(0<=n<=3)$ were included.

The full modal analysis process actually consisted of multiple computation steps:

1. TDMS data files produced by the NI 80-channel acquisition system were converted to MATLAB data arrays. Channel 73 was a copy of the zero-phase excitation signal set. The Fourier transform of this reference channel signal was used to phase-normalize the Fourier transforms of the 72 microphone channels and to select the spectral components corresponding to the seven-excitation frequencies. The cross-spectral matrix was computed for these seven components only, and the "condensed" phase-normalized spectra and cross-spectral matrices were equalized from tables created during the microphone calibration process and stored for use in the subsequent modal analyses. 
2. Spatial Fourier transforms were computed for each frequency and microphone row. The 24 microphones were equally spaced around each ring, so that complex circumferential mode amplitudes $A_{f m z}$ were recovered for $(-11<=m<=12)$ and plotted.

3. For each frequency and circumferential mode, the axial wavenumber was computed for each radial order $(0<=n<=3)$ based on cutoff ratio $\left(\eta=f l f_{\mathrm{co}}\right)$

$$
k_{z_{m n}}=\frac{2 \pi f}{c_{0}} \sqrt{1-\left(\frac{1}{\eta_{m n}}\right)^{2}}
$$

4. Transfer matrices were created for each frequency and circumferential mode from the geometric center of the three microphone rows.

$$
T_{f m}=\left[\begin{array}{llll}
e^{i k_{z m 0} \delta z_{1}} & e^{i k_{z m 1} \delta z_{1}} & e^{i k_{z m 2} \delta z_{1}} & e^{i k_{z m 3} \delta z_{1}} \\
e^{i k_{z m 0} \delta z_{2}} & e^{i k_{z m 1} \delta z_{2}} & e^{i k_{z m 2} \delta z_{2}} & e^{i k_{z m 3} \delta z_{2}} \\
e^{i k_{z m 0} \delta z_{3}} & e^{i k_{z m 1} \delta z_{3}} & e^{i k_{z m 2} \delta z_{3}} & e^{i k_{z m 3} \delta z_{3}}
\end{array}\right]
$$

where $\delta z$ is the axial distance to the microphone ring from the center.

$$
\left[\begin{array}{l}
A_{f m 1} \\
A_{f m 2} \\
A_{f m 3}
\end{array}\right]
$$

5. The complex amplitude vector was multiplied by the inverse of $T_{f m}$ to obtain the complex amplitudes of the radial modes. Note, however, that when the number of cut-on radial orders exceeds the number of microphone rows, the inverse of $T_{m}$ is actually a least squares approximation (using the MATLAB pinv function). For cases where only two or three radial order modes were cut on, the transfer matrix was truncated so that exact inversion was possible.

The alternative "beam-forming" approach was implemented in an attempt to better understand the effect of mode generation aliasing and mode separation aliasing that result from space-dictated too-sparse arrays of actuators and microphones. For each propagation mode $(-24<=m<=24)$ and $(0<=n<=3)$, a modal steering vector was computed

$$
S_{f m n M}=e^{i\left(m \theta_{M}+k_{z m n} \delta z_{M}\right)}
$$

where $M$ is the microphone identification index $(1<=M<=72)$. The standard beam-forming calculation was then used to determine the relative apparent strengths of the 196 trial modes

$$
B_{f m n}=\left\langle S_{f m n}\right\rangle\left[C_{f}\right]\left\langle S_{f m n}\right\rangle^{T}
$$

where $\left[C_{f}\right]$ is the $72 \times 72$ element cross-spectral matrix at frequency $f$ and $S_{f m n}$ is the 72 element steering vector for frequency $f$ and mode $(m, n)$.

For each mode, a conversion factor was computed to determine sound power from the outer wall sound pressure. 


$$
P_{\text {ratio }}=\frac{\int_{r_{1}}^{1} \psi(r)^{2} r d r}{\psi(1)^{2}}
$$

where $r_{1}$ is the ratio or inner to outer duct radius and

$$
\psi_{m, n}(r)=J_{m}\left(\pi \alpha_{m, n} r\right)+\beta_{m, n} Y_{m}\left(\pi \alpha_{m, n} r\right)
$$

the annular duct mode basis function. Sound Power Level was then computed

$$
L_{W m n}=10 \log \left(\frac{\pi\left(r_{\text {Outer }}^{2}-r_{\text {Inner }}^{2}\right)}{S_{\text {ref }}} \frac{p_{\text {Outer } m n}^{2}}{p_{\text {ref }}^{2}} P_{\text {ratio } m n} \sqrt{1-\left(\frac{1}{\eta_{m n}}\right)^{2}}\right)
$$

where $S_{\text {ref }}$ is $1 \mathrm{~m}^{2}$ and $p_{\text {ref }}$ is $20 \mu \mathrm{Pa}$.

\subsection{Testing}

\subsection{Configurations}

The general procedure was to first acquire data from the nacelle for each of the isolated (unshielded) configurations and then repeat the data acquisition for the same geometric positions of the nacelle but with the shield installed. The far field microphone array was traversed at the same set of spatial locations for both shielded and unshielded configurations Thus, each nacelle configuration (exhaust radiation minus shield with trailing edge, and inlet radiation minus shield with leading edge) was tested twice, once unshielded, and subsequently with the shield in place. A key point is that the array traverse was always in the same position relative to the edge or shield, or where it would be in the case of isolated nacelle testing.

Table I presents the model configurations tested. The acoustic signatures used for the results reported in this paper were termed the HWB. These are set acoustic signals with frequency and modal content based on a proposed turbofan engine and with a parametric variation in. Table II lists the frequency content based on the rating points of the proposed N2A turbofan engine and provides the modal structure for the first three harmonics based on the rotor-stator count and engine speed.

Acoustic time histories were acquired from a 13-microphone array. The microphone spacing on this array was $3 \mathrm{in}$. for a total span of $36 \mathrm{in.}$ The seventh microphone (center) was the position reference and at $Y=0$ tracked the model centerline. Far-field traverses were taken over four planar sweeps. Two horizontal planes were at approximately 7.5 and 10.1 nacelle diameters above the model. The two vertical planes were on one side of the model ( 6 and 12 diameters). The array was then shifted by 33 or 66 in. in the $+Y$ or $-Y$ direction and another planar sweep acquired. This shift resulted in overlapping data from two microphones. Thus the total lateral span acquired was $168 \mathrm{in.} \mathrm{The} \mathrm{resolution} \mathrm{between} \mathrm{traverse} \mathrm{stops}$ was varied, with a finer resolution closer to the model. Figure 20(a) depicts the traverse plane locations relative to the model. Figure 20(b) illustrates the variable spacing in the Y direction of the array stops. Photos of the model installed in the ATL are in Figure 21. 
TABLE I.-MODEL CONFIGURATIONS TESTED

(a) Exhaust nacelle (with and without trailing edge) (8)

\begin{tabular}{|c|c|}
\hline$x / d$ & $\theta$ \\
\hline 3.14 & $0^{\circ}$ \\
2.5 & $0^{\circ}, 22.5^{\circ}, 45^{\circ}, 60^{\circ}$ \\
1.5 & $0^{\circ}$ \\
0.0 & $0^{\circ}$ \\
-0.5 & $0^{\circ}$ \\
\hline
\end{tabular}

(b) Inlet nacelle (with and without trailing edge) (2)

\begin{tabular}{|c|c|}
\hline$x / d$ & $\theta$ \\
\hline 2.5 & $45^{\circ}$ \\
4.0 & $45^{\circ}$ \\
\hline
\end{tabular}

(c) Exhaust nacelle and verticals (with and without trailing edge) (4)

\begin{tabular}{|c|c|}
\multicolumn{2}{|c|}{$x / d=2.5 ; \theta=0^{\circ}$} \\
\hline$x_{\text {vert }} / d$ & $\delta$ \\
\hline 0.90 & $10^{\circ}$ \\
1.41 & $10^{\circ}$ \\
0.90 & $30^{\circ}$ \\
1.41 & $30^{\circ}$ \\
\hline
\end{tabular}

Note: Exhaust nacelle at $x / d=2.5, \theta=0^{\circ}$ is considered the baseline configuration.

TABLE II.-PROPOSED N2A TURBOFAN FIRST THREE HARMONIC FREQUENCIES AND STATOR INTERACTION MODES OF RATING POINTS

\begin{tabular}{|c|c|c|c|c|c|}
\hline Condition & $\mathrm{BPF}$ & $\begin{array}{c}\text { BPF-full } \\
\text { scale, } \\
\mathrm{Hz}\end{array}$ & $\begin{array}{c}\text { BPF-model } \\
\text { scale, } \\
\mathrm{Hz}\end{array}$ & $\begin{array}{l}\text { Modes, } \\
m, n_{\text {range }}\end{array}$ & Cut-off ratio \\
\hline \multirow[t]{3}{*}{ Approach } & 1 & 423 & $\mathrm{a}^{\mathrm{a}} 7286$ & ${ }^{\mathrm{b}}(-5,0)$ & 1.64 \\
\hline & 2 & 845 & 14571 & $(10,0: 2)$ & 1.26 \\
\hline & 3 & 1268 & 21857 & $(8,0: 3)$ & $3.15 / 2.22 / 1.55 / 1.13$ \\
\hline \multirow[t]{3}{*}{ Cut-back } & 1 & 826 & 14247 & $\mathrm{~b}(-5,0: 2)$ & $3.20 / 1.85 / 1.12$ \\
\hline & 2 & 1653 & 28493 & $(10,0: 4)$ & $3.34 / 2.47 / 1.88 / 1.42 / 1.12$ \\
\hline & 3 & 2479 & ${ }^{\mathrm{c}} 42470$ & ---------- & --------------------------------------------- \\
\hline \multirow[t]{3}{*}{ Takeoff } & 1 & 1117 & 19254 & $\mathrm{~b}_{(-5,0: 3)}$ & $4.32 / 2.51 / 1.51 / 1.05$ \\
\hline & 2 & 2233 & 38508 & $(10,0: 6)$ & $4.52 / 3.34 / 2.54 / 1.92 / 1.51 / 1.24 / 1.05$ \\
\hline & 3 & 3350 & c57762 & --------- & 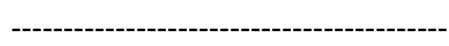 \\
\hline
\end{tabular}

${ }^{\mathrm{a}}$ Erroneously tested at $7,268 \mathrm{~Hz}$

${ }^{\mathrm{b}} m=-5$ tested arbitrarily for cutoff BPF

${ }^{\mathrm{c}}$ Frequency too high to control 
(H1)

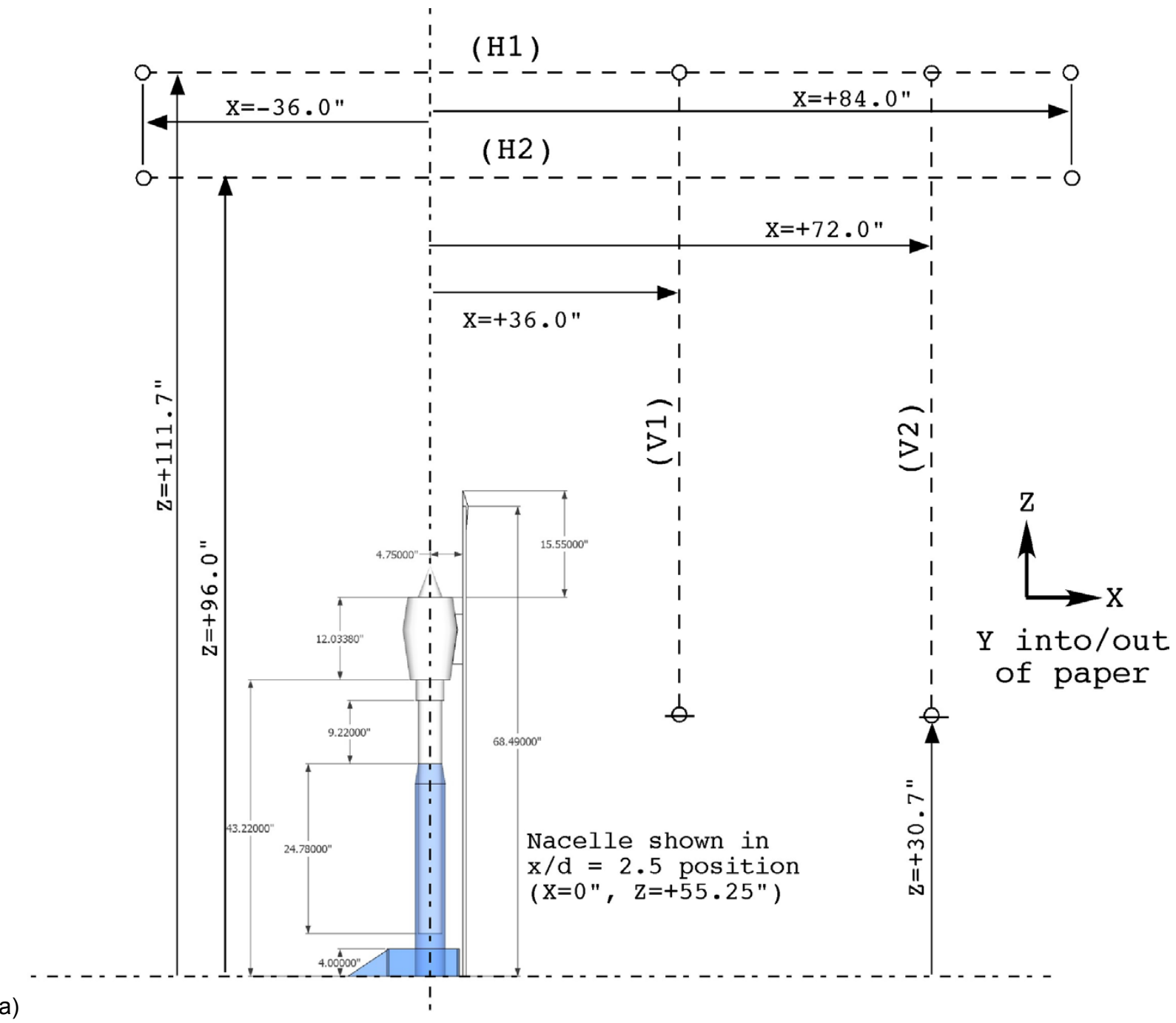

array is 13 mics; $36 "$ wide; 3 " between mics

$\mathrm{X}$ or $\mathrm{Z}$

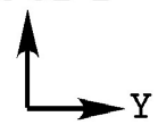

\section{In $\mathrm{X}-\mathrm{Y}$ plane:}

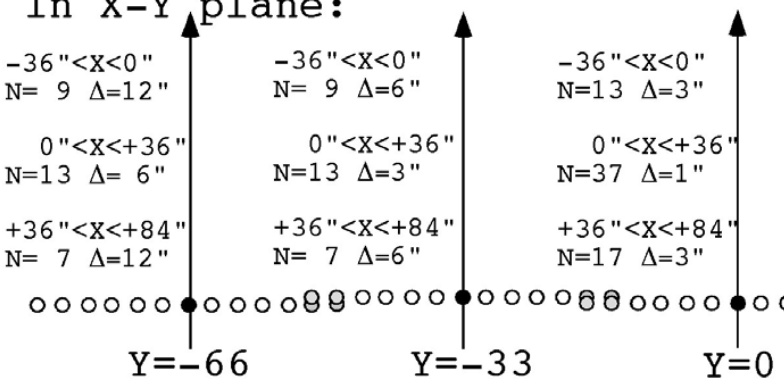

overlapped

center mic

$O$ mics

S

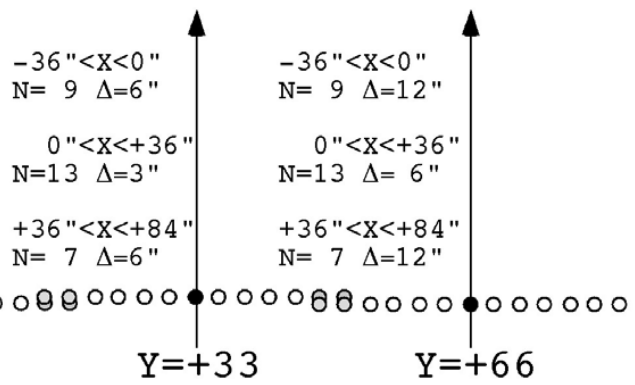

In Z-Y plane:

(b)

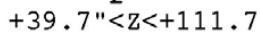

$\mathrm{N}=7 \quad \Delta=12 "$

$+33.7^{\prime \prime}<\mathrm{Z}<+111$

$\mathrm{N}=14 \Delta=6 "$

$30.7^{\prime \prime}<\mathrm{Z}<+111$ $\mathrm{N}=28 \Delta=3 "$

$\mathrm{N}=14 \Delta=6 "$

$+39.7^{\prime \prime}<\mathrm{Z}<+111.7^{\prime}$

Figure 20.-(a) Traverses for acoustic data, and (b) Resolution and spacing for X-Y and Z-Y microphone array sweeps. 


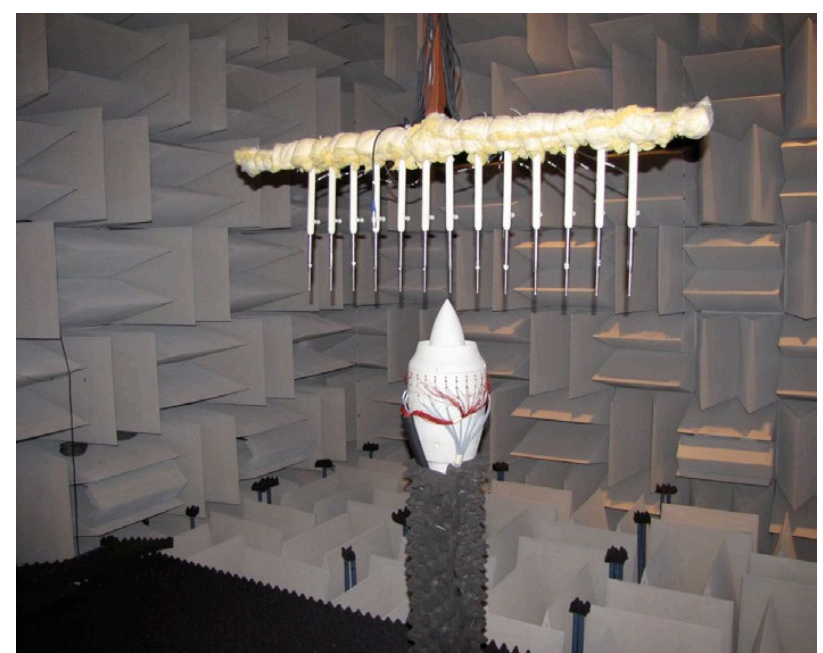

(a)

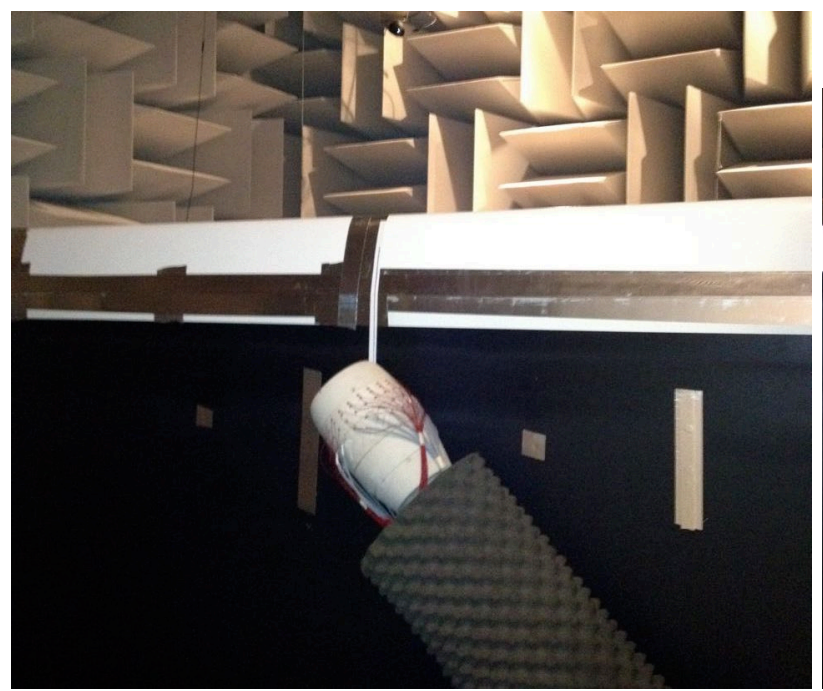

(c)

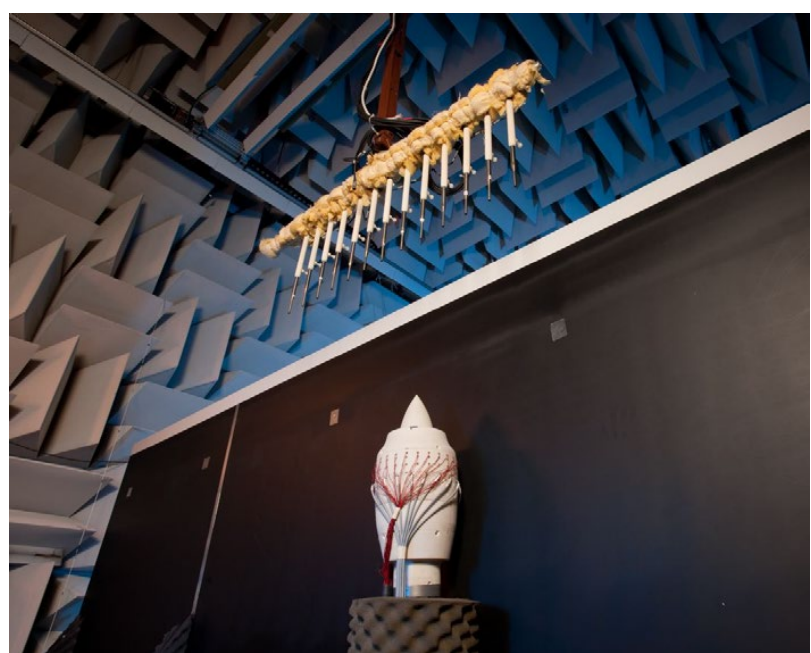

(b)

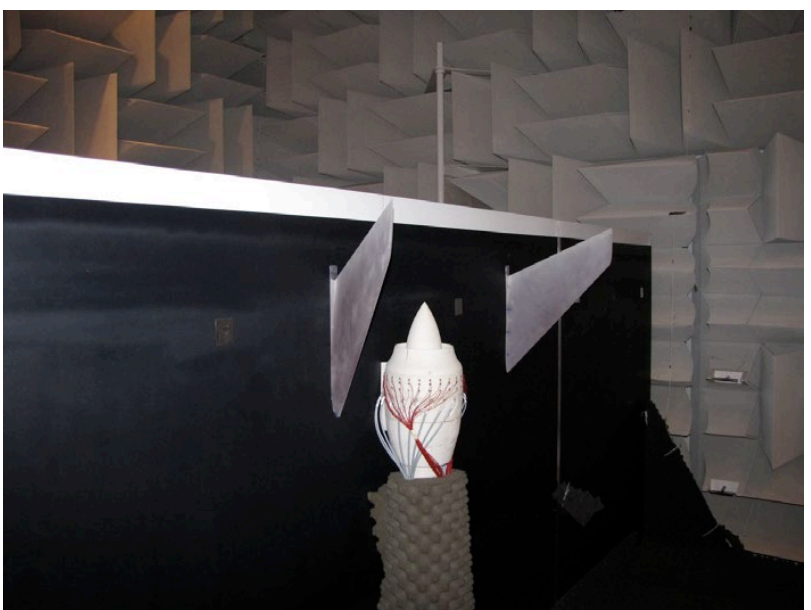

(d)

Figure 21.-(a) Isolated UCFANS exhaust configuration installed in ATL. (b) UCFANS exhaust configuration adjacent to shield with trailing edge. (c) UCFANS inlet configuration adjacent to shield with leading edge. (d) UCFANS exhaust configuration adjacent to shield with trailing edge and verticals mounted in position $x_{v e r} / d=1.41, \delta=10^{\circ}$.

\subsection{Reduced Data}

A significant amount of data was acquired for HWB shielding estimates. Reported herein is a selection of the reduced data, encompassing select changes in the parametric study. The data include the in-duct relative modal decomposition and sound pressure level (SPL) contour plots of the traverse planes. The SPL contour planes were also integrated to obtain noise power level (PWL) - (actually partial power to be used for comparison purposes). These are done for both the isolated nacelle and the nacelle with wing (shield) cases. In addition, deltas between the isolated nacelle/nacelle + wall configurations are presented to obtain shielding values. The convention will be to denote a reduction in PWL due to shielding as a negative number (cooler colors on the contour plots). A qualitative analysis is provided herein, as the objective is to assess the character of the system. 


\subsubsection{In-Duct Modal Analysis From Wall Microphone Data}

The in-duct modal signatures as discussed in Section 3.5.2 were further processed to estimate in-duct modal sound power levels. Absolute calibration of the in-duct microphones was determined by in-situ comparison with a laboratory microphone using the electrostatic actuator fitted with point source coupler horn. For each circumferential/radial mode amplitude from the basic separation and the basic functions for each mode, the modal pressure-squared distribution was integrated over the annular duct area, divided by $\rho c$ and multiplied by the group-velocity Mach number. For the code-validation set of test modes, the computed in-duct sound powers agree within approximately $2 \mathrm{~dB}$ of the power levels determined from integration of the far field array measurement.

Figure 22 to Figure 25 show the mode decomposition results for representative signal generation. Each of the first three figures is the result of computing based on a number of radial modes ranging from 2 to 4 . For convenience, the engine operating point reference and target mode are shown for each. Referring to Table II, the only case where the two-mode inversion applies (see Figure 22) would be Approach BPF, with one mode propagating, $(-5,0)$. Mode $(-5,0)$ is clearly dominant in the inversion, with $(-5,1)$ over $10 \mathrm{~dB}$ down. The only significant spurious mode is $(8,0)$ at $2 \mathrm{~dB}$ down, which could be an intermodulation distortion result from the strongly excited Approach 2BPF and 3BPF modes at $m=-10$ and $m=8$, which are potential excitation aliases for 18 -actuator inner and outer subgroups.

The three-mode inversion should be applicable for Approach 2BPF and Cutback BPF, which are nearly the same frequency, 14,571 and 14,247 Hz respectively. Referring to Figure 23 it is seen that although the Cutback BPF displays significant content at mode $(-5,1)$, it is tertiary in indicated strength compared to modes at $m=7$ and $m=-11$. These two spurious modes are aliases of each other for an 18-element actuator array, but have not obvious relationship to the $m=-5$ target mode. The beam-forming result shown in Figure 25 also shows the strong contribution at $m=-11$, and a secondary peak at $m=13$, which is an alias of -11 for 24 microphones. Target mode $m=-5$ is again tertiary. Approach 2BPF displays a clearly dominant mode $(8,0)$, secondary $(8,1)$ and tertiary $(2,0)$. The dominant $m=8$ is an alias of the target mode $m=-10$ for an 18-element actuator array. Since all 36 actuators were driven for this example, it is unclear why the aliased mode is predominant. In this case, the beam-form result confirms the dominance of $m=8$, but the radial components are reversed in relative strength and target mode $m=-10$ is tertiary.

Four-mode inversion applies to Approach $3 \mathrm{BPF}$ and Takeoff BFF (21,857 and 19,254 Hz respectively). As seen in Figure 24, the Approach BPF shows a significant contribution at $(8,1)$ but it is 7 to $8 \mathrm{~dB}$ weaker than three of the radials at $m=2$. In this case, the beam-form solution indicates dominance by $(-16,1)$ with $(8,2)$ only about $2 \mathrm{~dB}$ weaker. Note that $m=-16$ is an alias of $m=2$ for an 18-element actuator array and $m=-16$ is an alias of target $m=8$ for the 24-microphone array, so it is possible a compound alias effect produces the observed result. Take-Off BPF shows secondary strength at target mode $m=-5$, approximately $5 \mathrm{~dB}$ below spurious mode $m=-11$. The beam-forming result also shows significant target mode content, but dominance by $m=13$, which aliases to $m=-11$ in the microphone array and $m=-5$ in the 18 -element actuator array. Again, a compound aliasing effect is suggested.

Cutback and Takeoff $2 \mathrm{BPF}$ are predicted to comprise five and seven radial components each at mode $m=-10$, so that spuriously excited lower-order circumferential modes would include a much larger number of radial components than could be discriminated in either the inversion or the beam-forming process. In both cases, a low-order circumferential mode is strongly dominant, $m=0$ for the Take-Off case and $m=2$ for the Cutback case. Using the four-radial inversion, at Cutback 2 BPF, $m=8$ is secondary, and is an excitation alias of the target mode $m=-10$. The beam-forming result is dominated by $m=-22$, which aliases to $m=2$ in the microphone array. The beam-forming result also shows a strong contribution at $m=-16$, which is a microphone array alias of $m=8$, which in turn is an excitation alias of target mode $m=-10$. At Takeoff $2 \mathrm{BPF},(-10,2)$ and its excitation-alias $(8,2)$ are present, but weak compared to $(0,2),(0,0)$ and $(2,2)$. The beam-forming result shows moderate strength at $m=-22$ and $m=2$, and at the sequence $m=-16,-4,8,20$. These all may relate to the $m=-10$ target mode by aliasing combinations from the actuator and microphone arrays, but it is not clear why the actual target mode is nearly insignificantly weak compared to the spurious modes.

The difficulties encountered in analyzing the in-duct modal distribution with an extensive, but nonetheless sparse array of microphones together with generating the in-duct sound field with an array of 
actuators that is on the cusp to satisfying Nyquist limitations suggest that for actual radiated mode analysis, an external system may be preferable. Early in the UCFANS development, the OptiNav Array48 system with advanced Generalized Inverse processing was employed to test some of the CVM mode properties, with good indication of success. The in-duct system is effective for validating excitation consistency; modal distributions computed from data collected at several stages of production testing were nearly invariant.

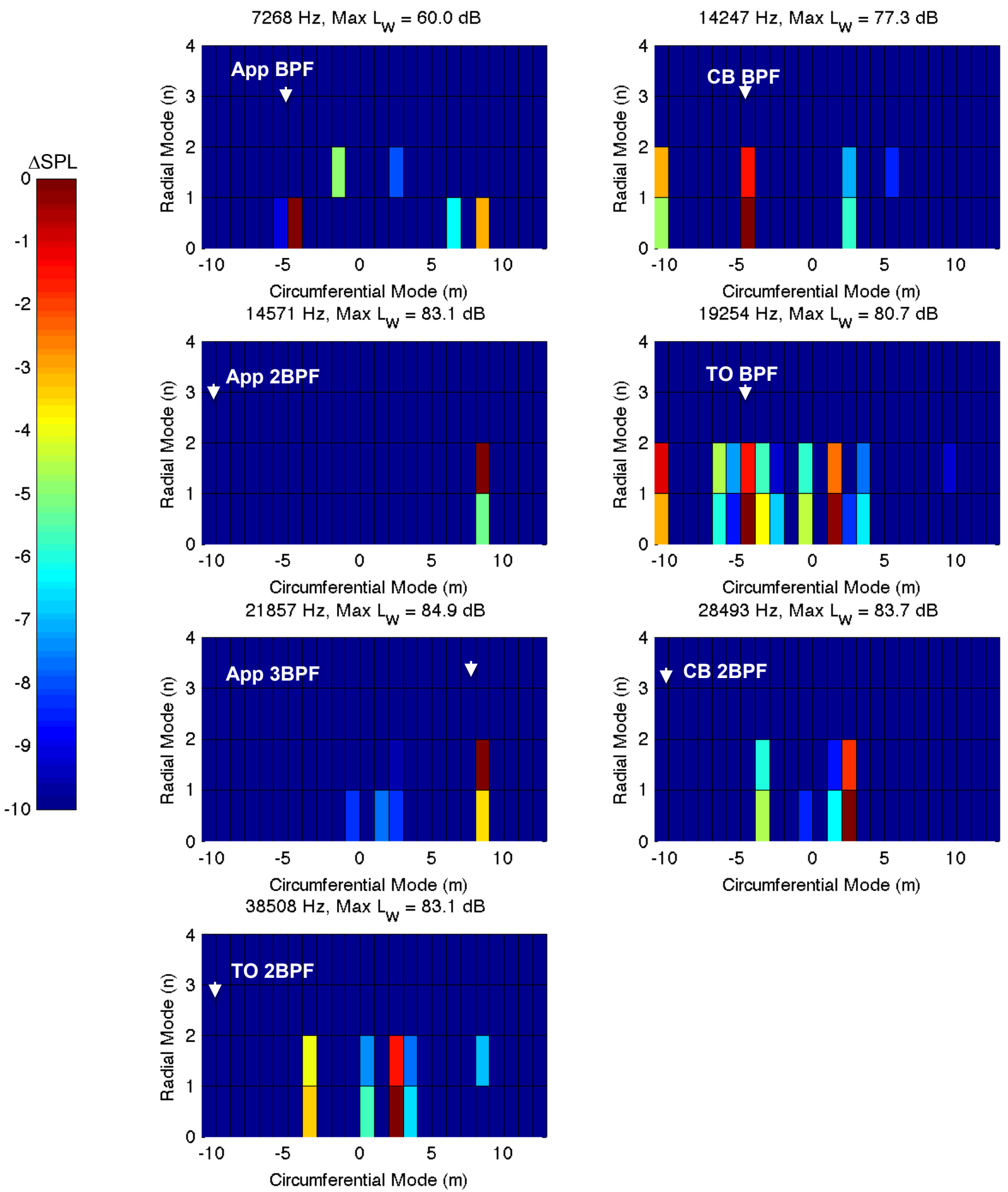

Figure 22.-HWB modal analysis (outer and inner actuators driven)-Two radials in inversion (refer to Table II for target modes). 

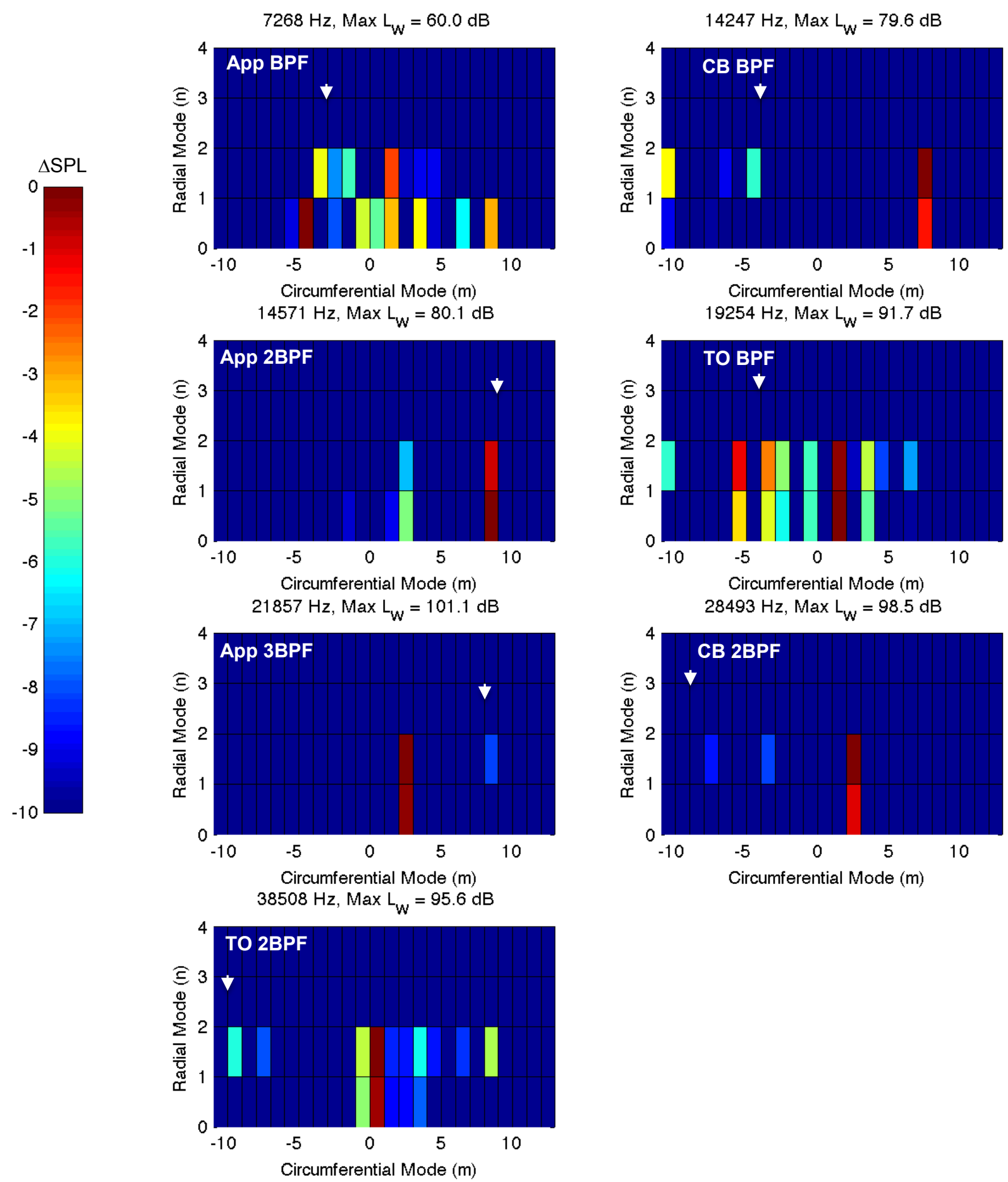

Figure 23.-HWB modal analysis (outer and inner actuators driven)-Three radials in inversion (refer to Table II for target modes). 

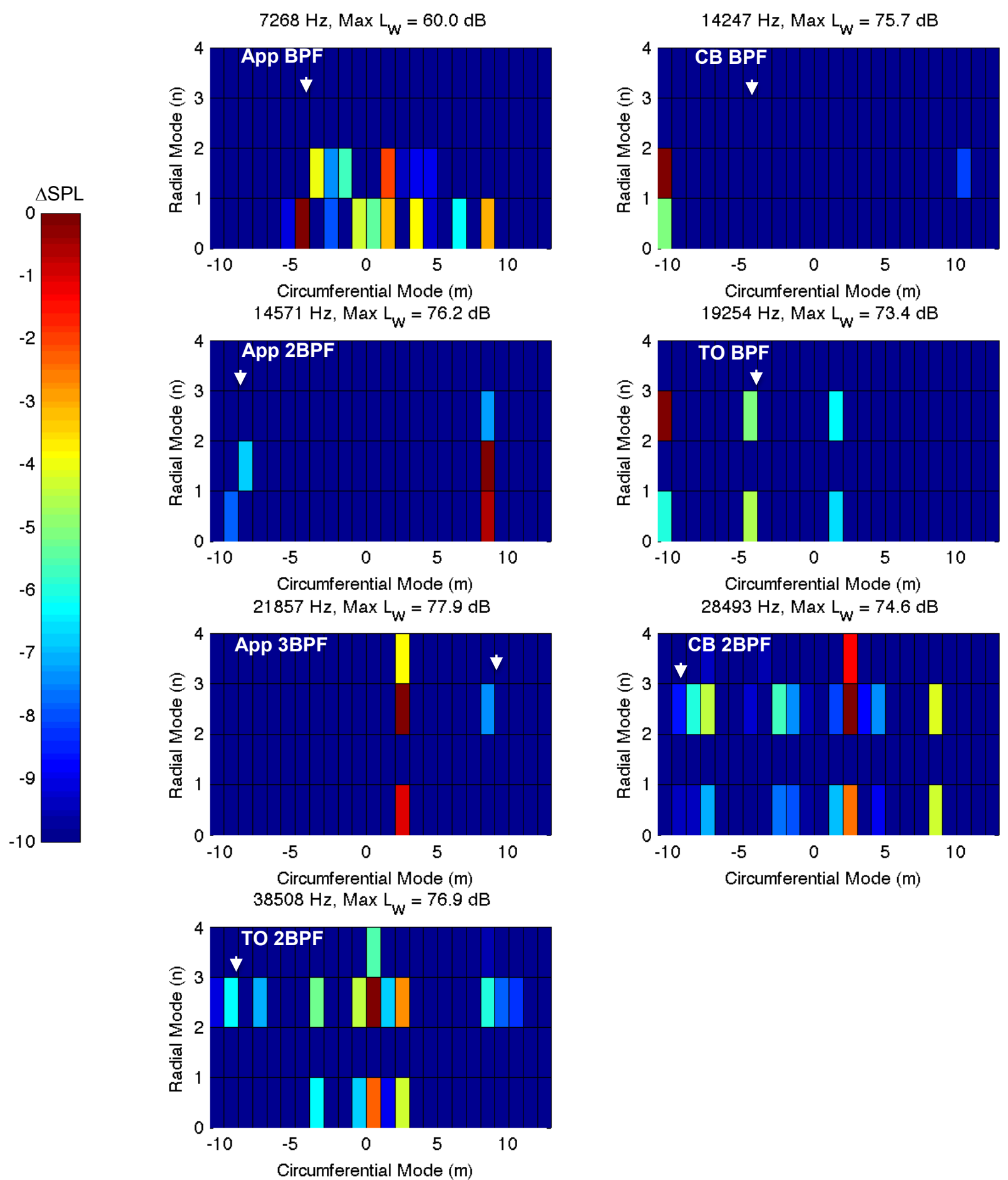

Figure 24.-HWB modal analysis (outer and inner actuators driven)—Four radials in inversion (refer to Table II for target modes). 

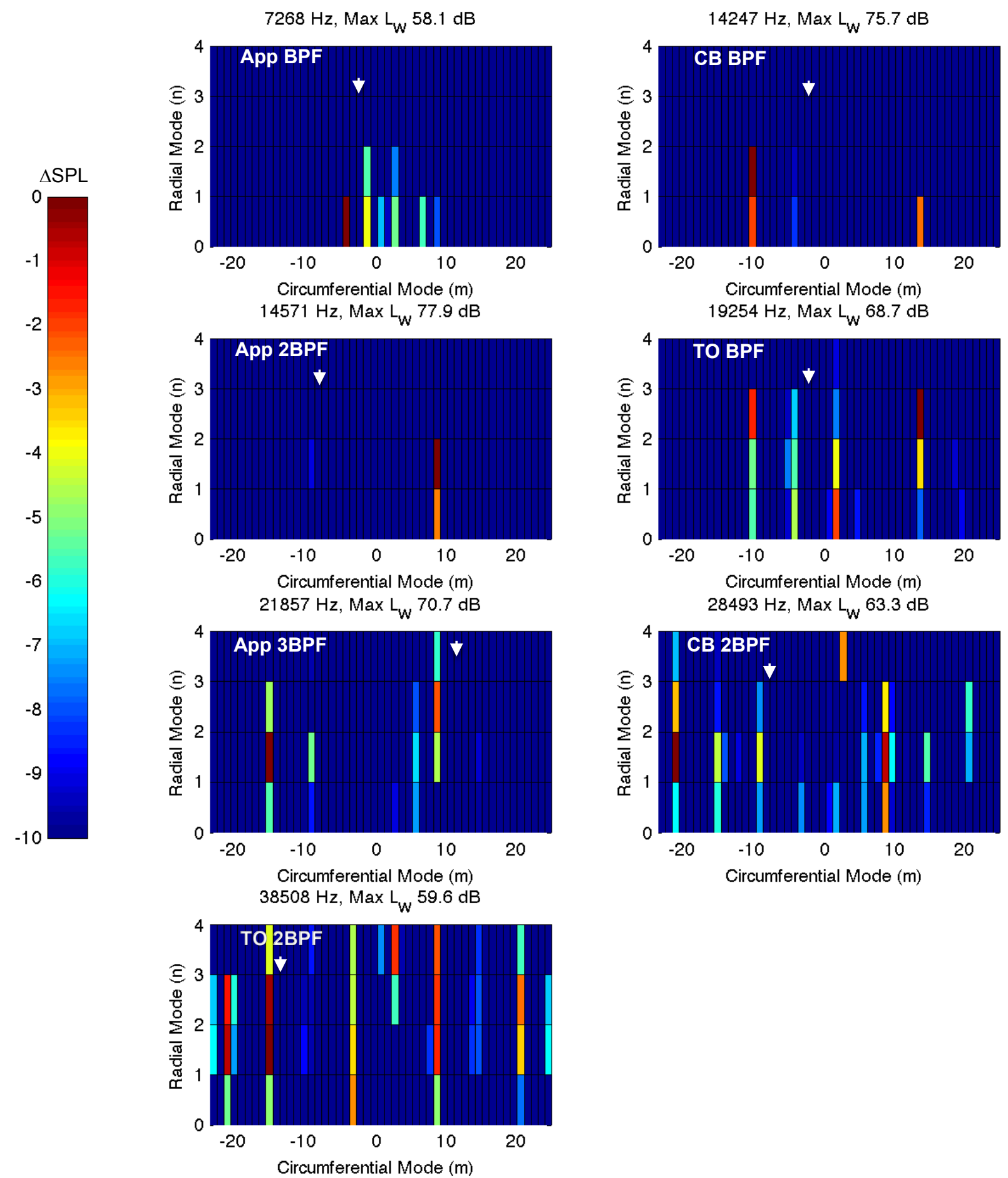

Figure 25.-HWB modal analysis (outer and inner actuators driven)—Beam-form solution (refer to Table II for target modes).

\subsubsection{Far-Field Array Contours From Nacelle Radiation}

Far-field noise data were acquired to document the acoustic engine signature interaction with the HWB plan-form (which could be due to shielding, reflection, and diffraction) and provide data for the development of noise propagation codes. Time series data were recorded at a $200 \mathrm{kHz}$ sample rate. A Kaiser window function ( $2^{14}$ points) was then applied and a Fourier transform used to convert the data to "as measured" spectra. Each spectrum was then corrected on a frequency-by-frequency basis for the individual 
microphone response and the effect of the grid cap (using calibration curves supplied by the manufacturer). Finally, the data were converted to a lossless condition by correcting for the atmospheric attenuation of propagating sound. Note that the data presented are at the measurement location and include the spherical spreading of sound. At this point, the tones of interest may be extracted from the overall spectrum.

Figure 26 to Figure 34 show the far-field SPL contours for a representative sample of the configurations tested at various traverse planes as described in Figure 20(a) and (b). For the contour plots, the nacelle centerline and radiation plane is at $(0,0)$. The wall, if present, will be at $X=+4.75$ in.

(a) SIGNAL 1 (Both Radial Driver Sets Acuated)
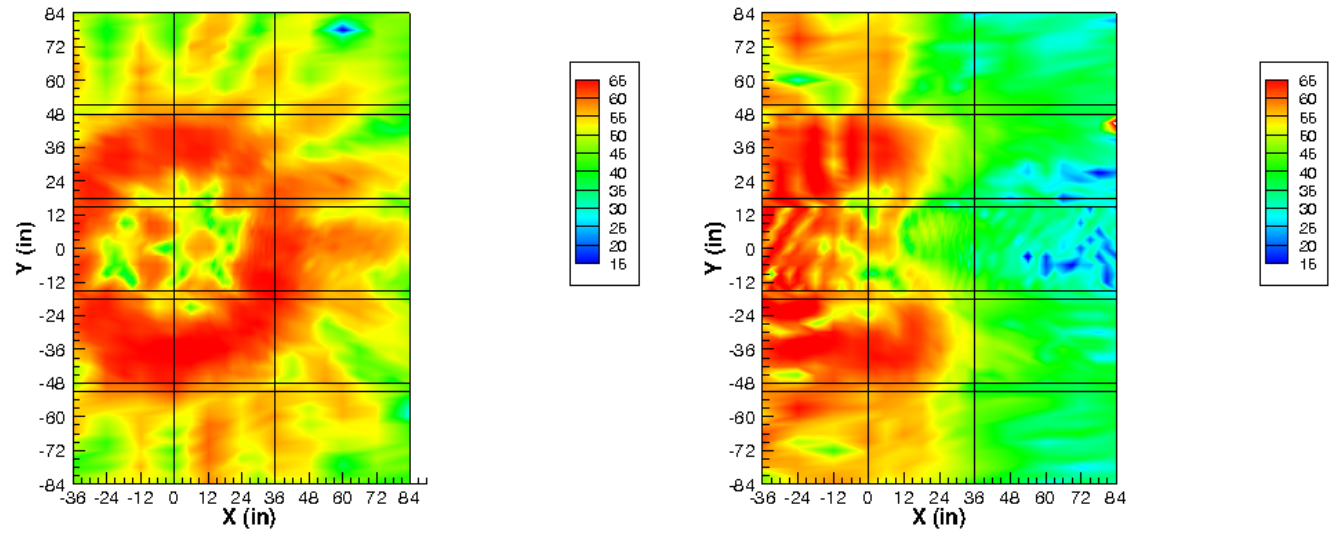

(b) SIGNAL 2 (Inner Radial Set Driver Actuated)
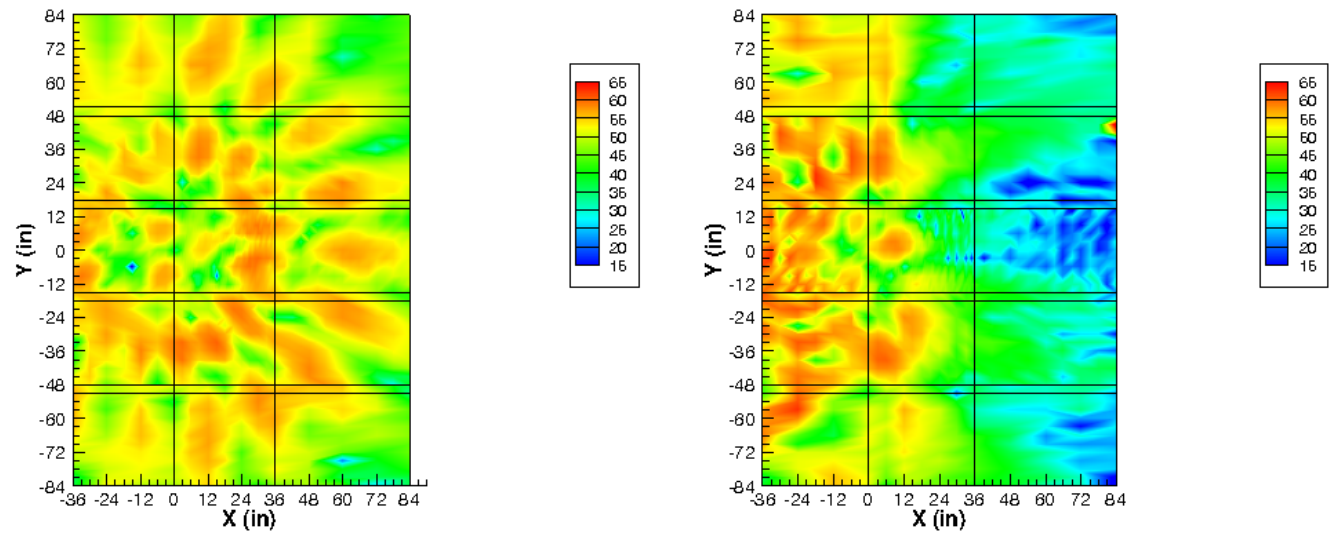

(c) SIGNAL 3 (Outer Radial Driver Set Actuated)
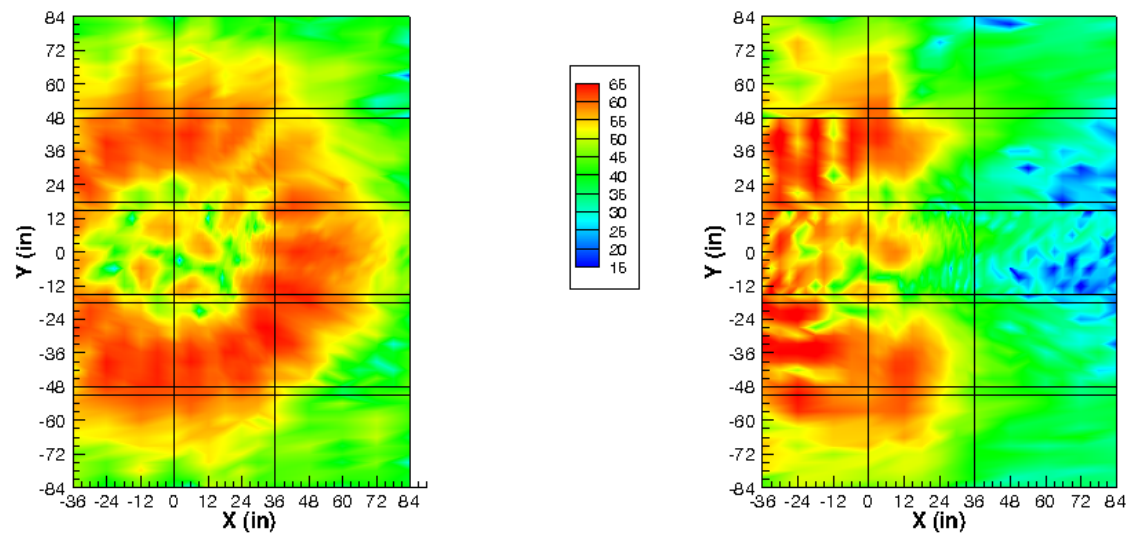

Figure 26.-Far-field SPL contour plot showing effect of radial signal. Left column is from isolated exhaust nacelle; right column is nacelle mounted on $2-\mathrm{D}$ wing with trailing edge. $x / d=2.5, \theta=0^{\circ}$, Approach, $2 \mathrm{BPF}, \mathrm{H} 1$ measurement plane. 
In general the plots show the SPL value in either an X-Y or Y-Z plane. In all cases the plane was fixed in space, and the position of the model adjusted to effect the orientation change. This necessitated the acquiring of data twice for each configuration: nacelle alone and nacelle plus wing/wall (for brevity the configuration with the nacelle mounted to the wing/wall will be referred to as the mounted-nacelle). In those pairs the location of the nacelle remained fixed in space. This allows for direct comparison between the no wall/wall configuration pairs - but when comparing between two different geometric configurations some discrepancy occurs as a result of the distance between the nacelle radiation and farfield traverse planes changes.

Figure 26 shows the effect of varying relationship of the signal to the radial actuators. Figure 26(a) is the result when the inner and outer actuators are both excited; Figure 26(b) and (c), the inner- and outerset of radial actuators are actuated independently, respectively. It appears that the combined set is a superposition of each set of radial actuators. Changing the relationship of the actuator signals has a clear influence on the far-field radiation. This is present in both the isolated nacelle radiation (left hand side) and the mounted-nacelle radiation patterns (right hand side).

Figure 27 and Figure 28 show the effect of varying the measurement planes (refer to Figure 20(a) and (b)). In each orientation, two planes were acquired. Figure 27 shows the two different horizontal measurement planes (think of them as planes in behind of the aircraft). Figure 28 shows the two different vertical measurement planes (think of them as planes below the aircraft). The modal structure is seen clearly in the horizontal planes, and as the distance increases from the nacelle the spherical spreading is apparent. The closest vertical plane to the nacelle captures the radiation of the bottom edge of the modal lobe, but as this distance increases, the bulk of the lobe is missed by the measurement plane.

Figure 29 shows the effect of varying the fan harmonic at the approach condition. This results in the primary circumferential mode changing per Table II. At BPF (Figure 29(a)) the far-field pattern is broken. This may be due to mode $m=5$ 's principal lobe angle radiating outside the measurement plane and the plane capturing higher-order spillover modes. At 2 BPF (Figure 29(b)) the increasing frequency corresponds to a higher cut-off ratio and a principal mode angle closer to the nacelle centerline and thus captured by the measurement plane. Finally, at 3 BPF (Figure 29(c)) multiple radials are cut-on, of which the lowest two are highly cut-on and are discernible in the far-field as seen by the two concentric circular patterns in the plot on the left.

Figure 30 shows the effect changing the rating point. Here the frequency will increase, but the circumferential mode order will remain constant while the number of radials increases with the higher rating point. $2 \mathrm{BPF}$ was chosen as the fan harmonic. The earlier mentioned principal lobe pattern is seen in the approach rating point (Figure 30(a)). As the rating increases to cutback (Figure 30(b)) the focus becomes narrower, and at takeoff (Figure 30(c)) there is significant energy at the centerline as well as very broad extent due to the high number of radials cut-on. It is interesting to observe that because of the narrowness of the beaming, the wing/wall does not block any of the lobe radiation at cutback and takeoff rating points. However, at approach the wing/wall reflects a significant portion of the lobe.

In Figure 31 we see changes in the acoustic interaction due to the wing/wall, as a result of varying the location of the nacelle exhaust exit plane relative to the trailing edge in terms of nacelle diameter $(x / d)$. The nominal condition 2BPF, approach, $x / d=2.5$ is repeated in Figure 31(b). At this location the mode radiation pattern is blocked and reflected to the left (above the aircraft). A little spill over to the right (under the aircraft) can be detected. Moving the nacelle further from the trailing edge $(x / d=3.14$, see Figure 31(a)) provides even greater shielding and lower spill over in to the shaded zone. On the other hand, overhanging the nacelle, $(x / d=-0.5$, see Figure $31(\mathrm{c}))$ results in almost no shielding. Note that Figure 31 presents a case where the distance between the nacelle and the measurement plane changes as the nacelle is moved relative to the wing and hence the measurement plane. 
(a) Horizontal Plane 1
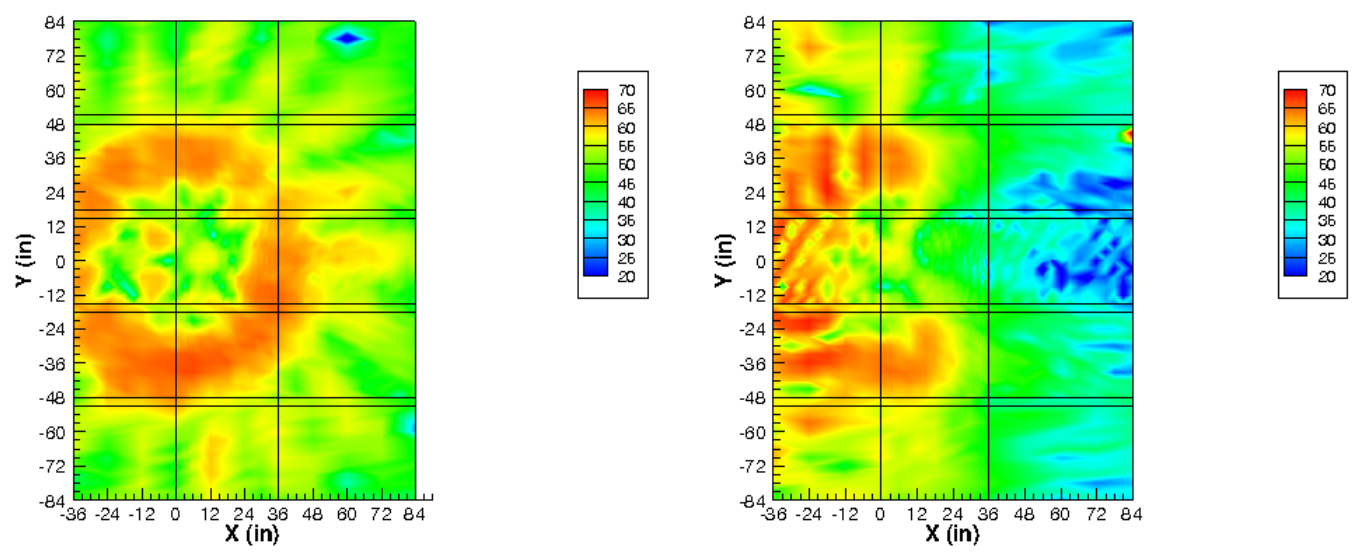

(b) Horizontal Plane 2
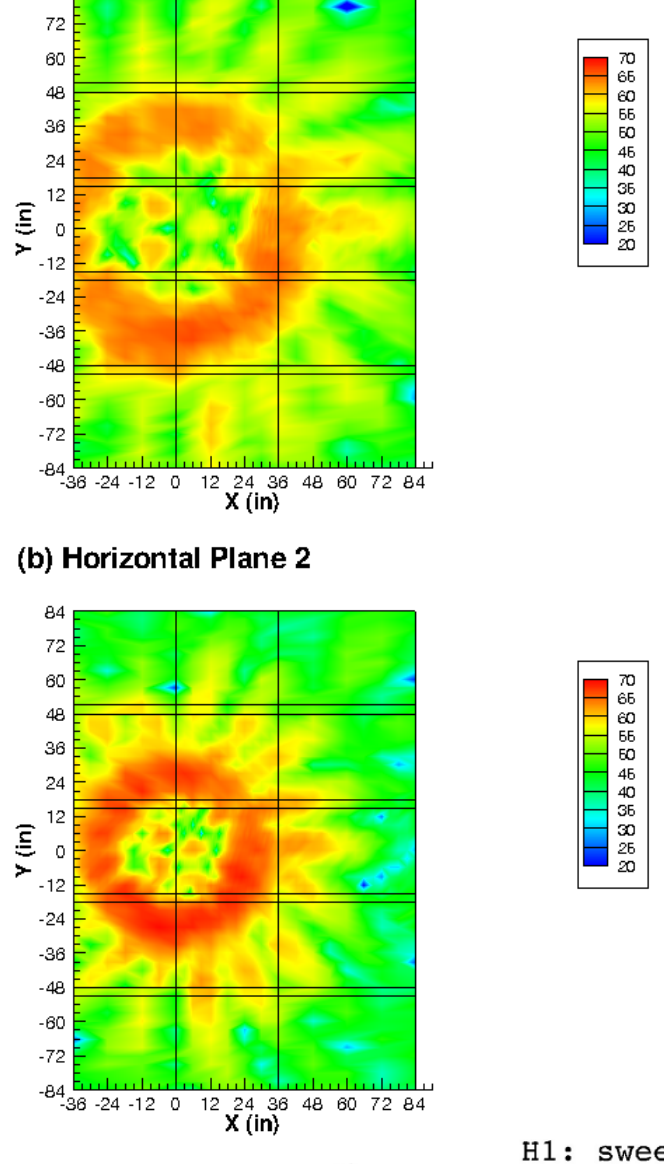

\section{政}
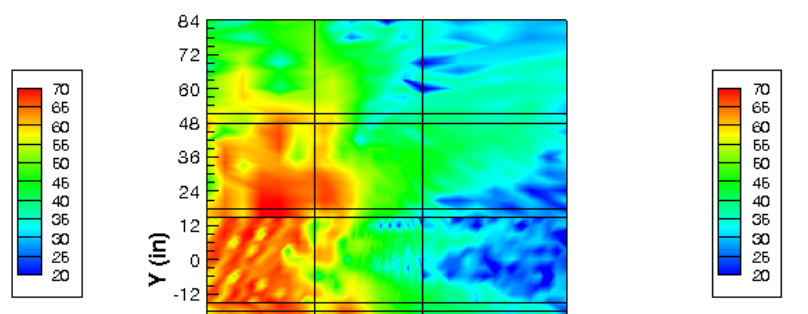

H는

H2: sweep @

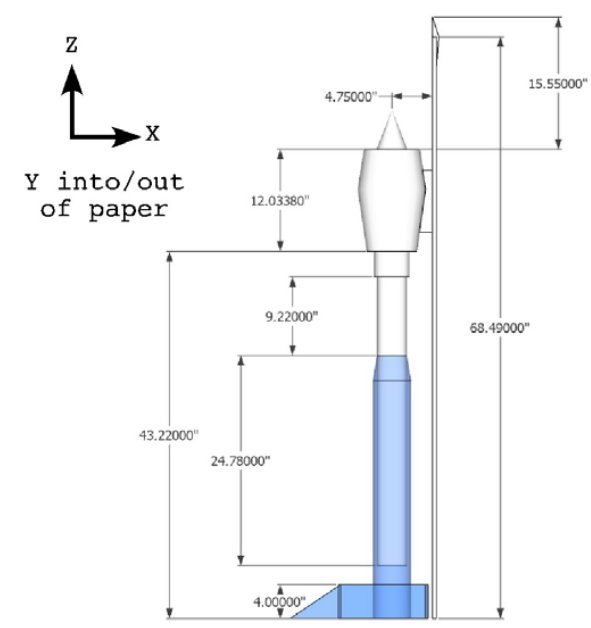

Figure 27.-Far-field SPL contour plot at different horizontal measurement planes $(\mathrm{H} 2, \mathrm{H} 1)$. Left column is from isolated exhaust nacelle; right column is nacelle mounted on 2-D wing with trailing edge. $x / d=2.5, \theta=0^{\circ}$, Approach, 2PBF, Signal 1. 
(a) Vertical Plane 1
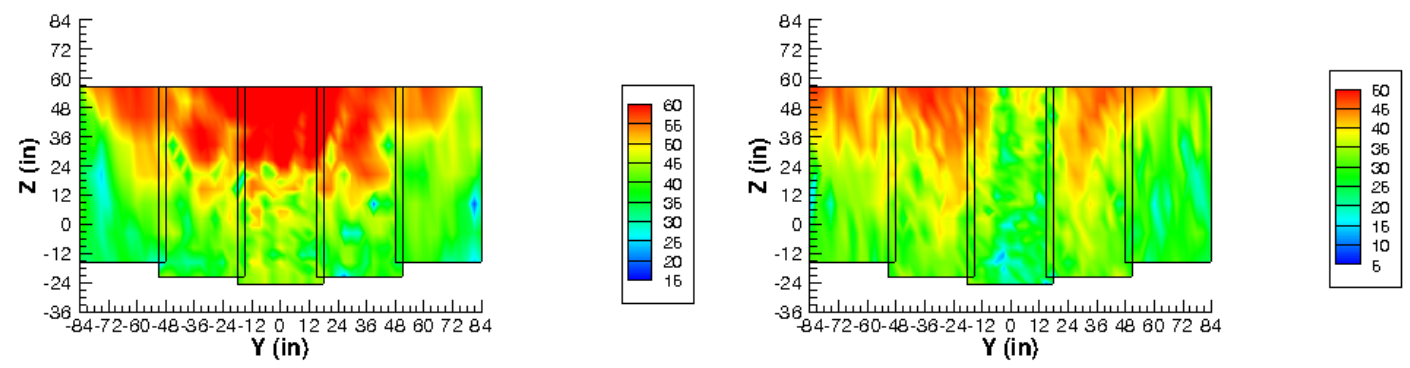

(b) Vertical Plane 2
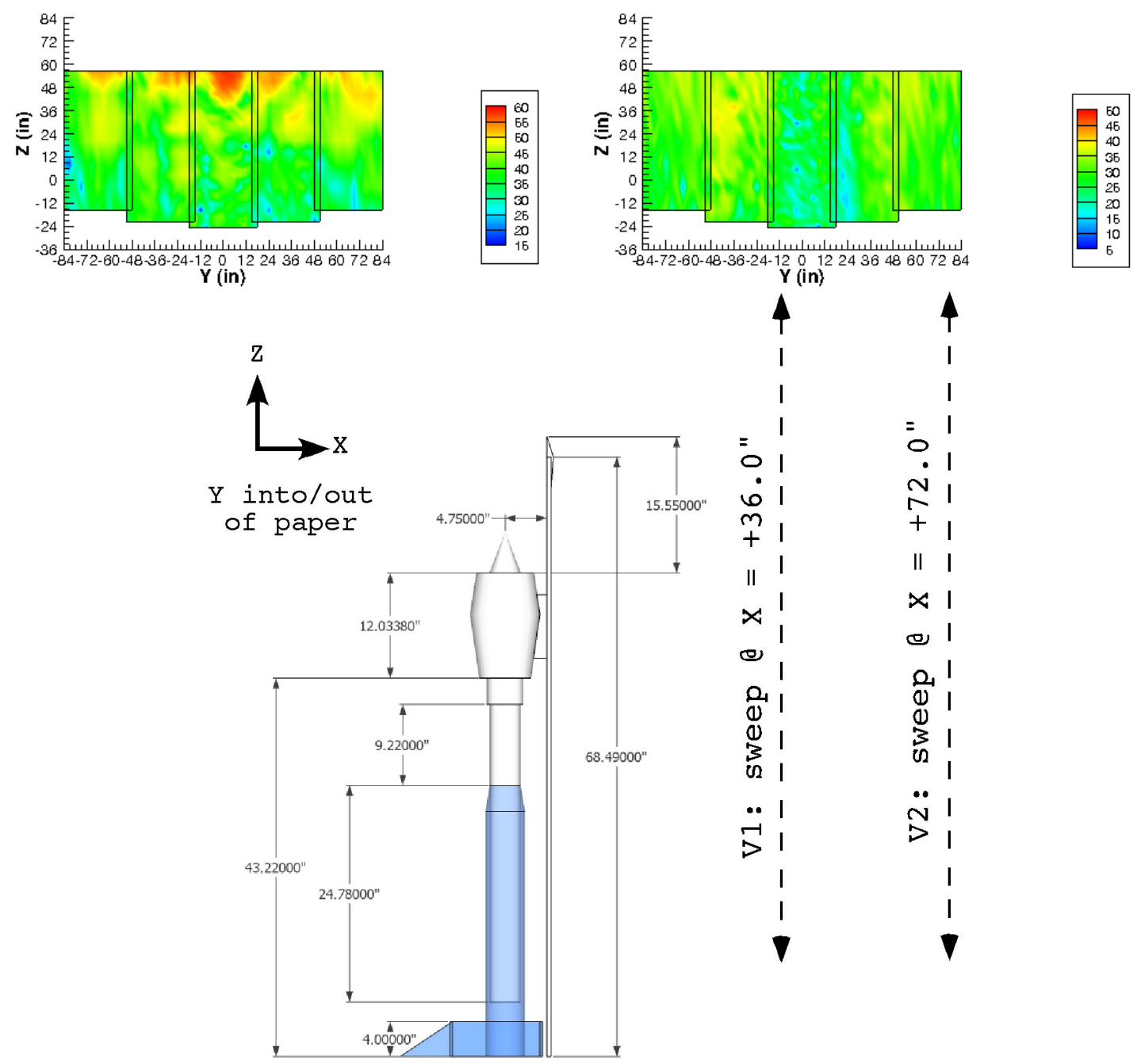

Figure 28.-Far-field spl contour plot at different vertical measurement planes (V2,V1). Left column is from isolated exhaust nacelle; right column is nacelle mounted on $2-\mathrm{D}$ wing with trailing edge. $x / d=2.5, \theta=0^{\circ}$, Approach, $2 \mathrm{PBF}$, Signal 1 
(a) $1 \times B P F$
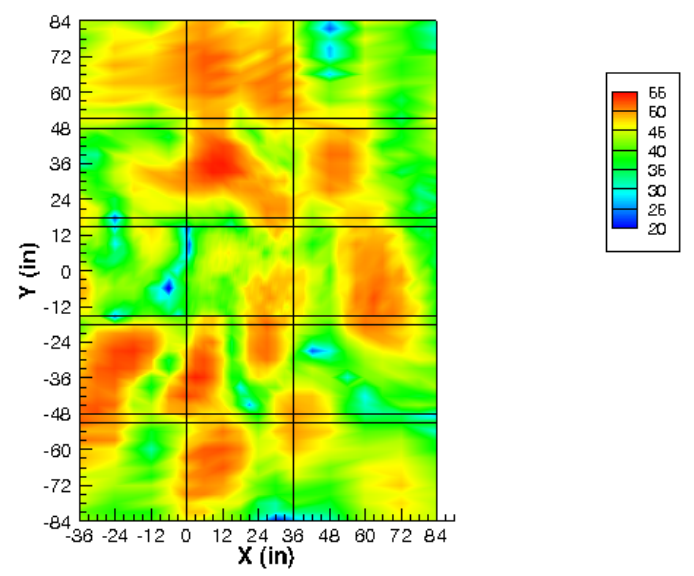

\section{(b) $2 \times B P F$}
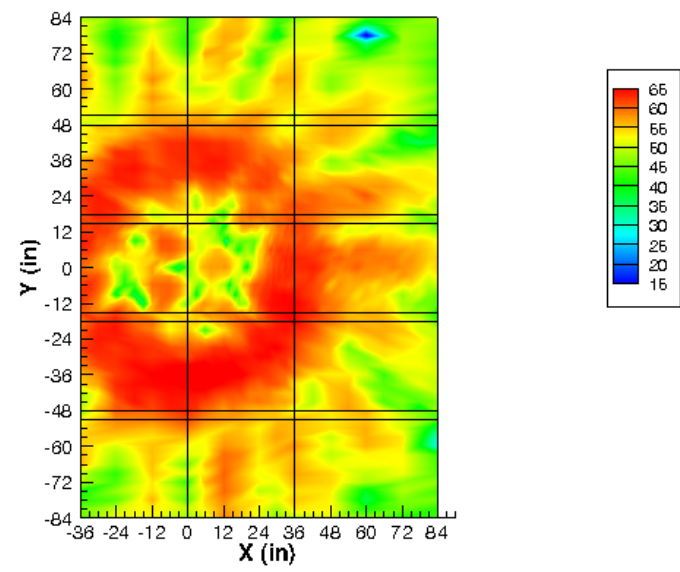

\section{(c) $3 \times B P F$}

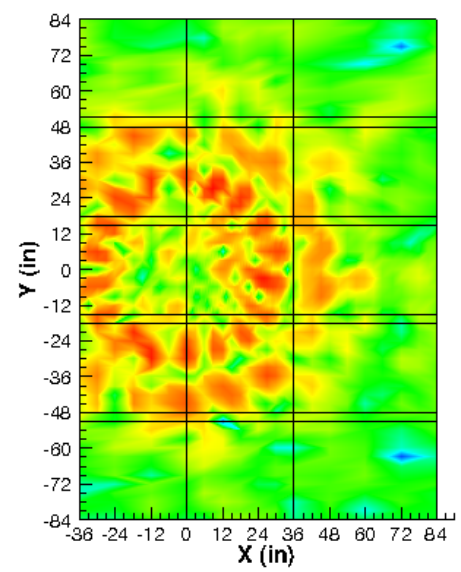

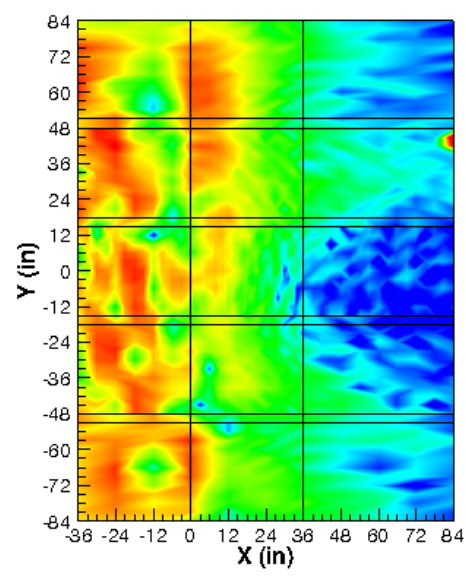

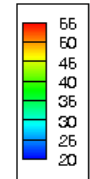

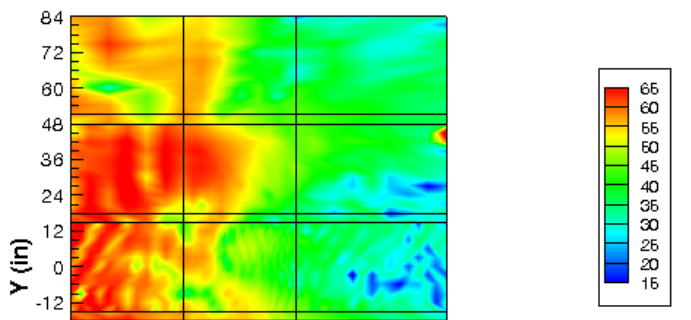

Figure 29.-Far-field SPL contour plot at first three fan harmonics (1 BPF, 2 BPF, 3 BPF). Left column is from isolated exhaust nacelle; right column is nacelle mounted on 2-D wing with trailing edge. $x / d=2.5, \theta=0^{\circ}$, Approach, $\mathrm{H} 1$ measurement plane, Signal 1. 


\section{(a) Approach}
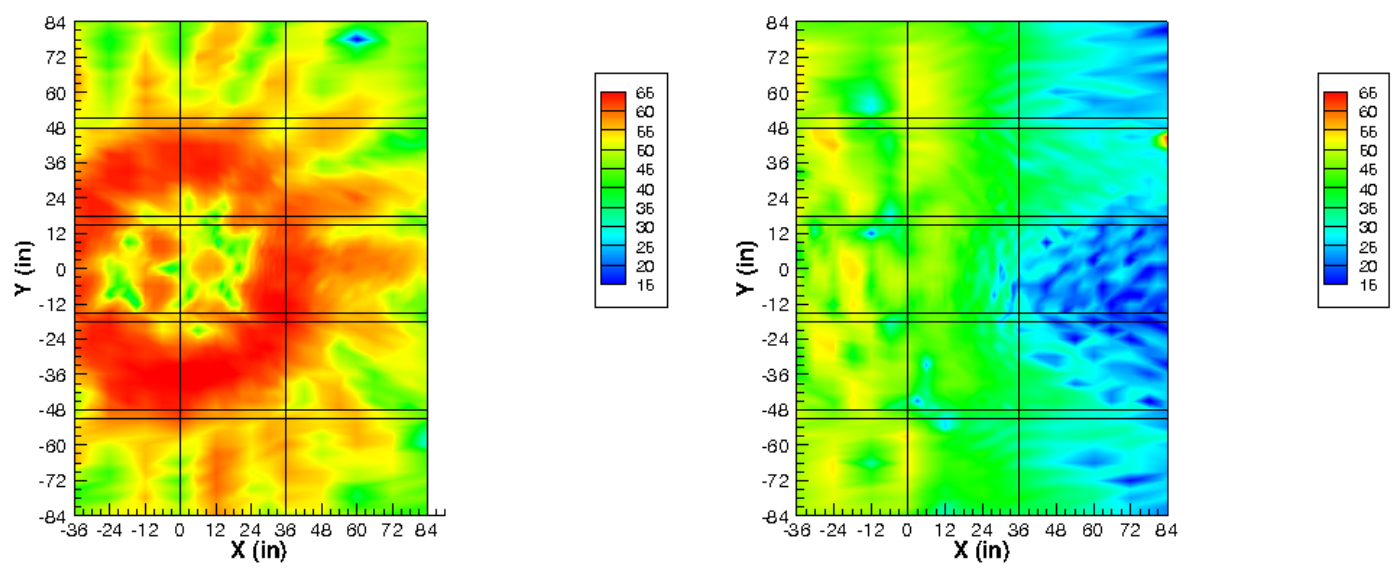

\section{(b) Cutback}
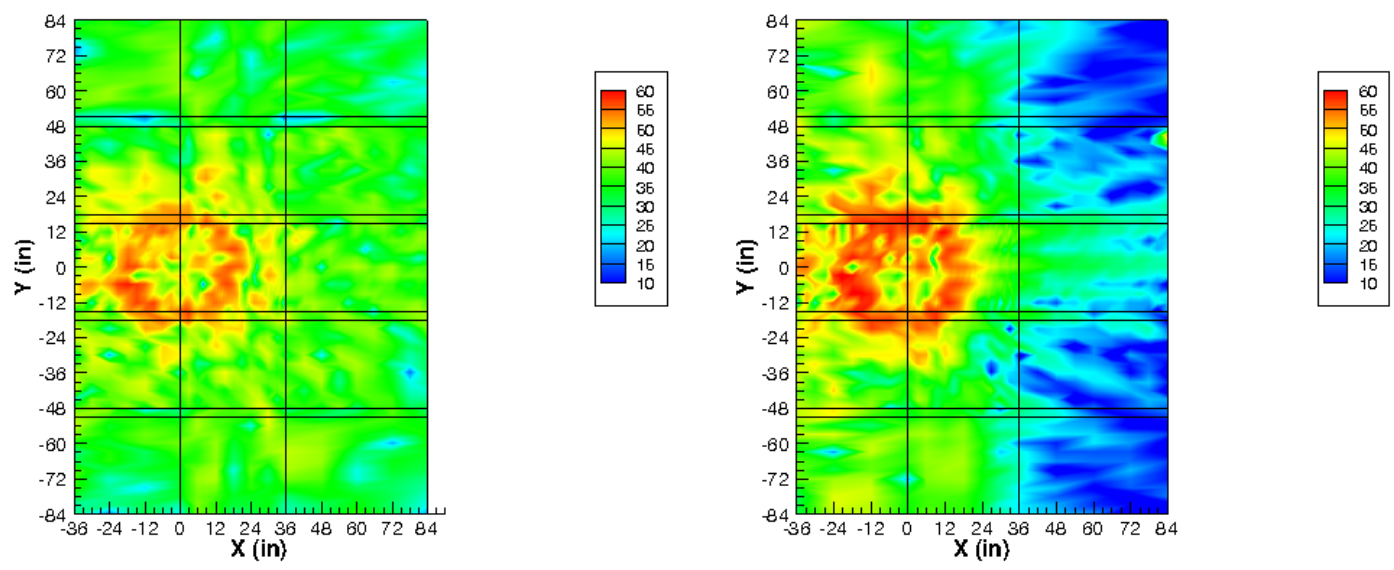

\section{(c) Takeoff}
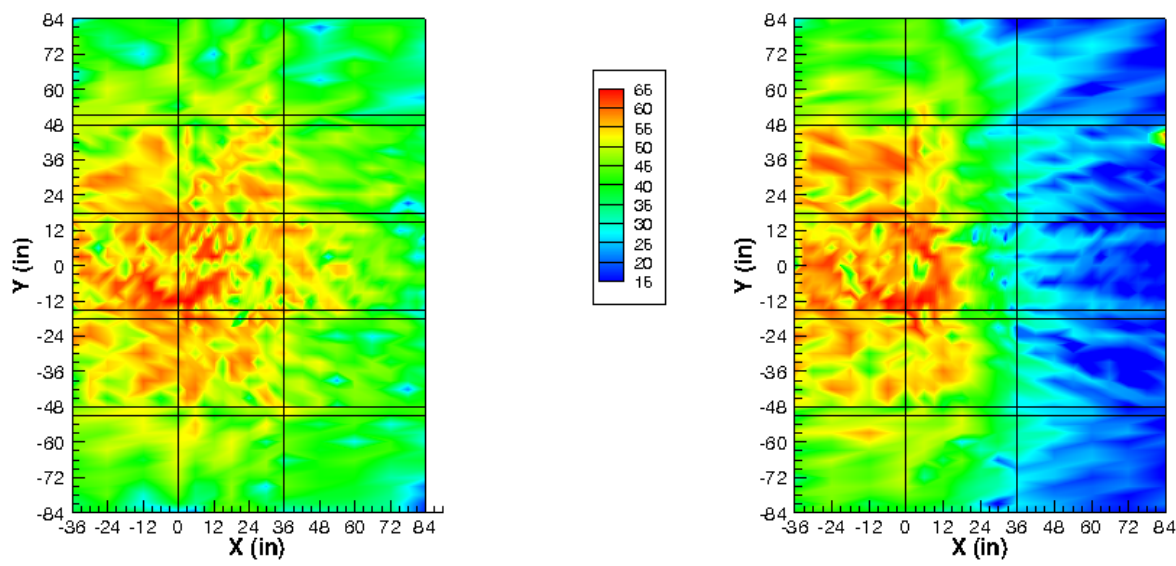

Figure 30.-Far-field SPL contour plot at rating points (Approach, Cutback, Takeoff). Left column is from isolated exhaust nacelle; right column is nacelle mounted on 2-D wing with trailing edge. $x / d=2.5, \theta=0^{\circ}, 2 \mathrm{BPF}, \mathrm{H} 1$ measurement plane, Signal 1. 


\section{(a) $x / d=3.14$}

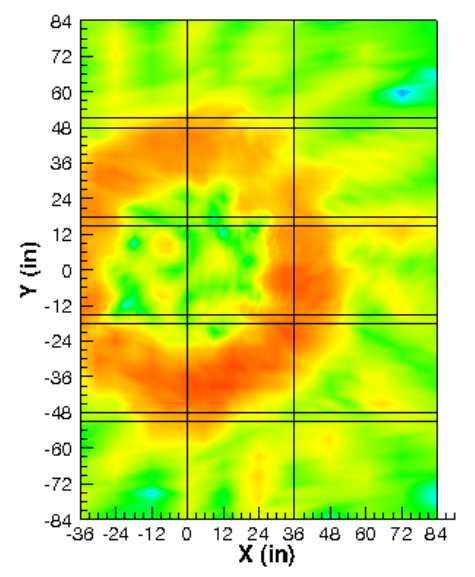

(b) $\mathrm{x} / \mathrm{d}=\mathbf{2 . 0}$

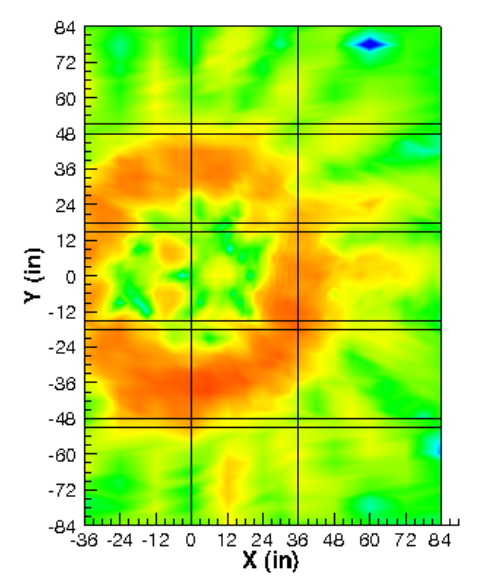

(c) $x / d=-0.5$

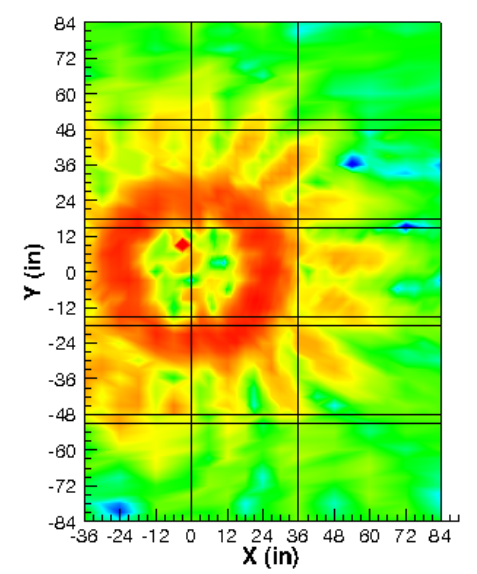

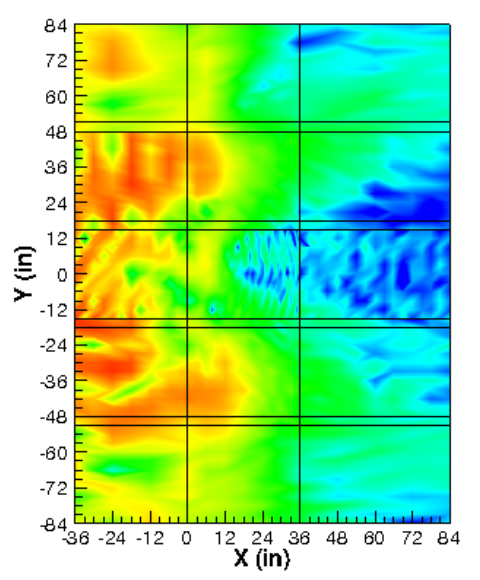
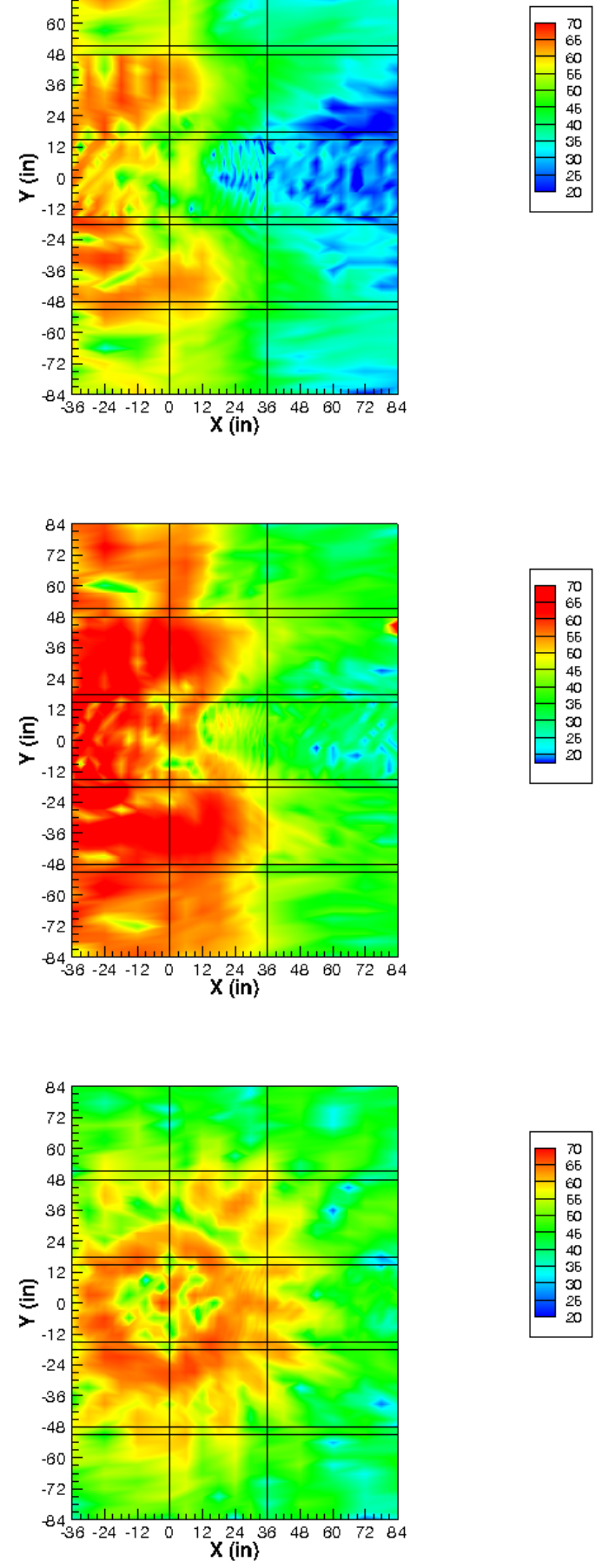

Figure 31.-Far-field SPL contour plot at various $x / d$ Locations $(3.14,2.5,-0.5)$ Left column is from isolated exhaust nacelle; right column is nacelle mounted on 2-D wing with trailing edge. $\theta=0^{\circ}$, Approach, $2 \mathrm{BPF}, \mathrm{H} 1$ measurement plane, Signal 1.

Recall the model wing was simplified to a 2-D form in order to provide a simpler modeling case for code validation. To parametrically account for the fact that the aircraft will have a 3-D plan-form, the angle of the nacelle centerline relative to trailing edge line was varied $\left(0^{\circ}\right.$ is defined as the center-line 
perpendicular to the trailing edge line). The far-field SPL radiated from the exhaust nacelle at $0^{\circ}, 22.5^{\circ}$, and $45^{\circ}$ angles in shown Figure 32(a), (b), and (c), respectively. Again the measurement plane is fixed in space, so the first effect noticed is the plane is now slicing through the lobe at higher angles as the nacelle angle changes. This has an effect on the shielded contours in the same manner, in that at the highest angle only a portion of the lobe is captured.

(a) Nacelle Angle $=0$ deg

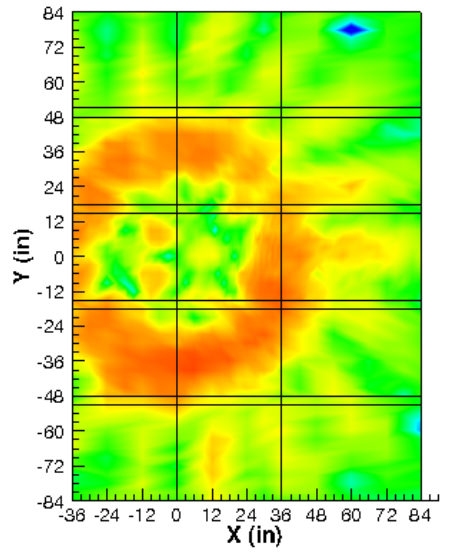

(b) Nacelle Angle $=\mathbf{2 2 . 5} \mathrm{deg}$

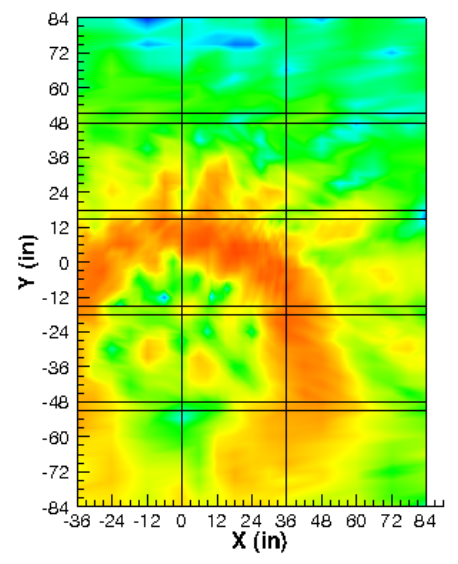

(c) Nacelle Angle $=\mathbf{4 5} \mathrm{deg}$

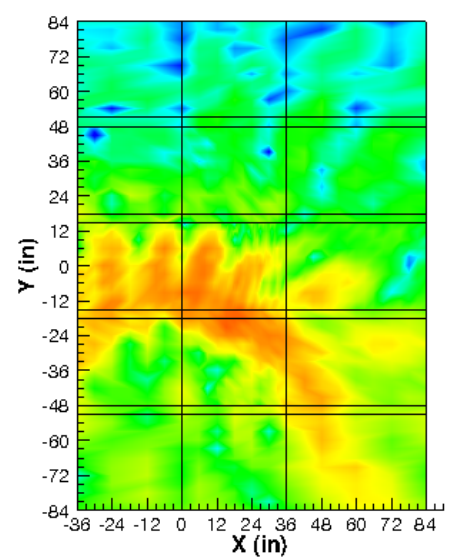

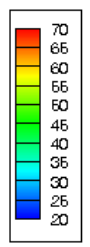
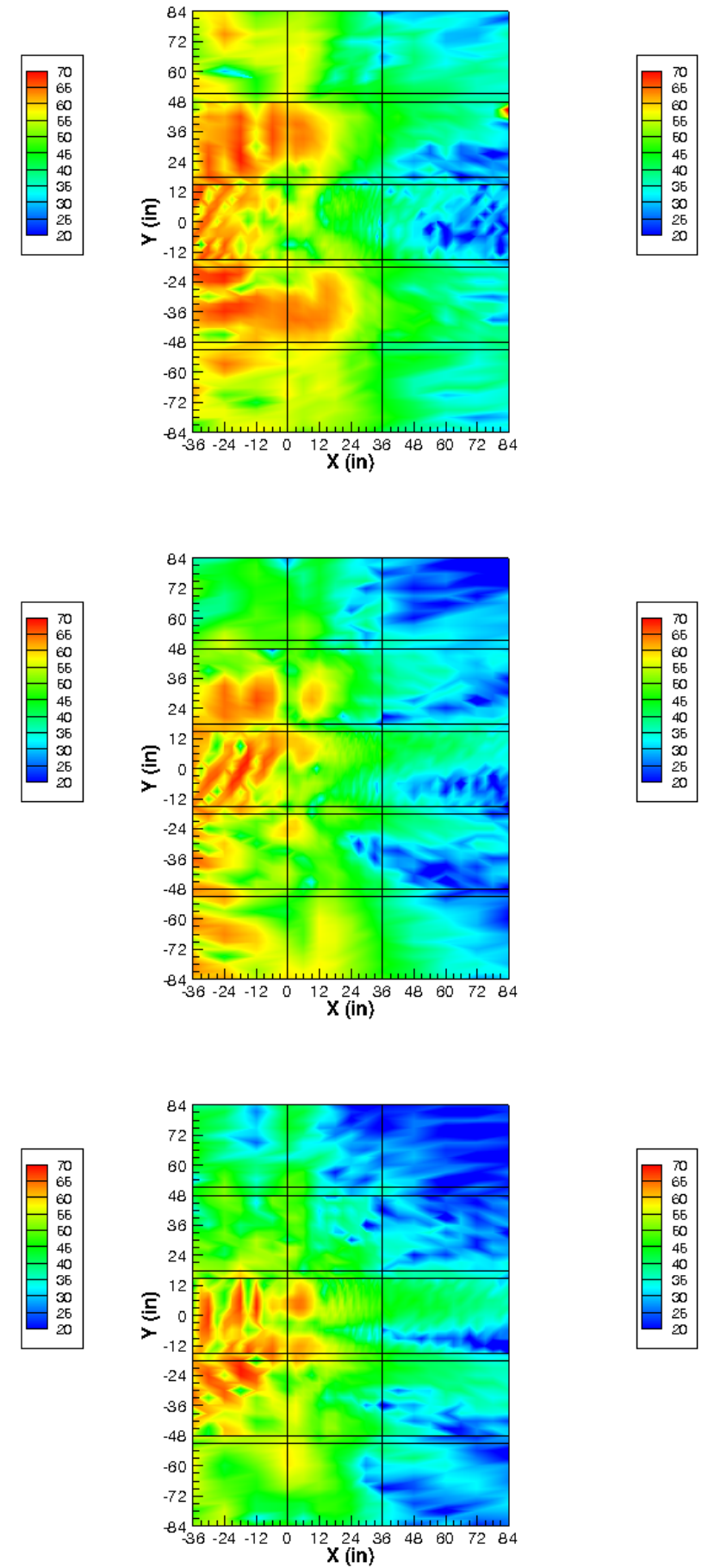

Figure 32.-Far-field SPL contour plot at various nacelle angles $\left(\theta=0^{\circ}, 22.5^{\circ}, 45^{\circ}\right)$. Left column is from isolated exhaust nacelle; right column is nacelle mounted on 2-D wing with trailing edge. $x / d=2.5$, Approach, $2 \mathrm{BPF}, \mathrm{H} 1$ measurement plane, Signal 1. 
The effect on acoustic interaction due to the addition of a pair of verticals is illustrated in Figure 33 . Two cant angles and two axial positions of the verticals were tested. In terms of the plot, the single nacelle was on the positive side of the $\mathrm{Y}$-axis. Additional shielding on in the $(-\mathrm{X},-\mathrm{Y})$ quadrant occurs as a result of the presence of the verticals. It appears as if the vertical opposite of the nacelle reflects a portion of the radiation onto the $(+\mathrm{X},+\mathrm{Y})$ quadrant of the contour.

(a) Exhaust Nacelle Alone

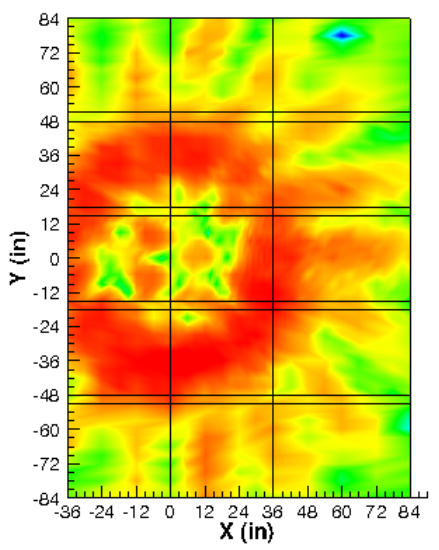

(b) $x$-vert $/ \mathrm{d}=0.90 \quad$ Angle $=10 \mathrm{deg}$

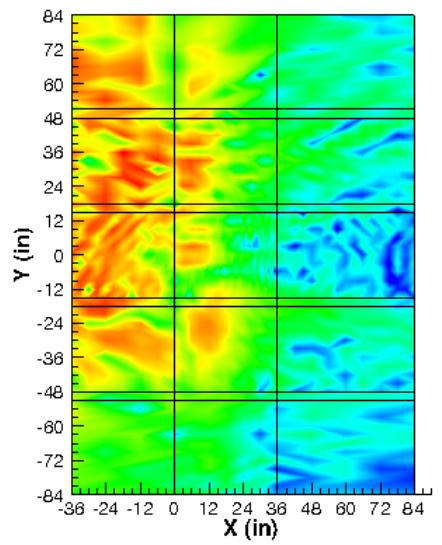

(c) $x$-vert/d=1.41 Angle=10 deg

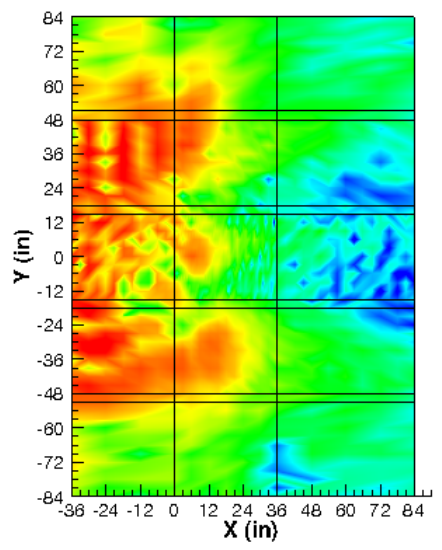

(d) Exhaust Nacelle + Trailing Edge Wing Only

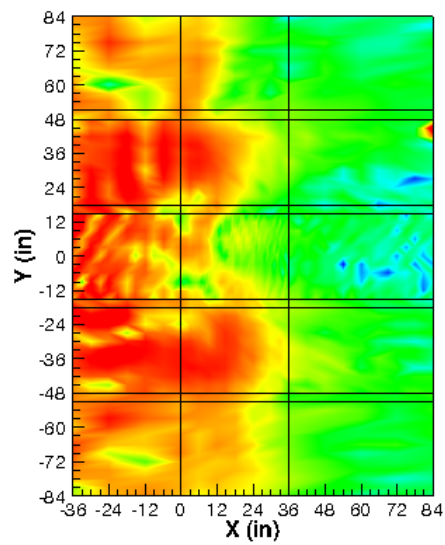

(e) $x$-vert $/ \mathrm{d}=0.90$ Angle $=30 \mathrm{deg}$

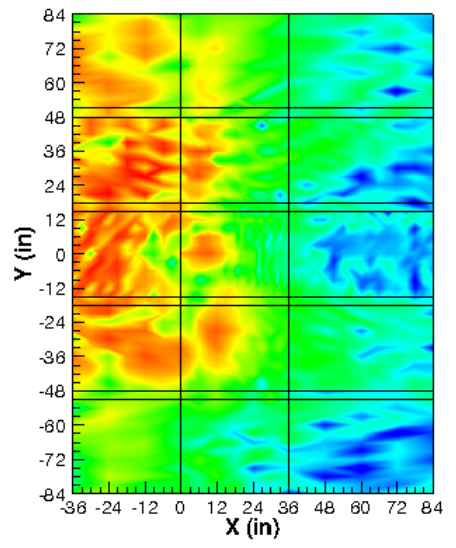

(f) $x$-vert/d Angle=30 deg
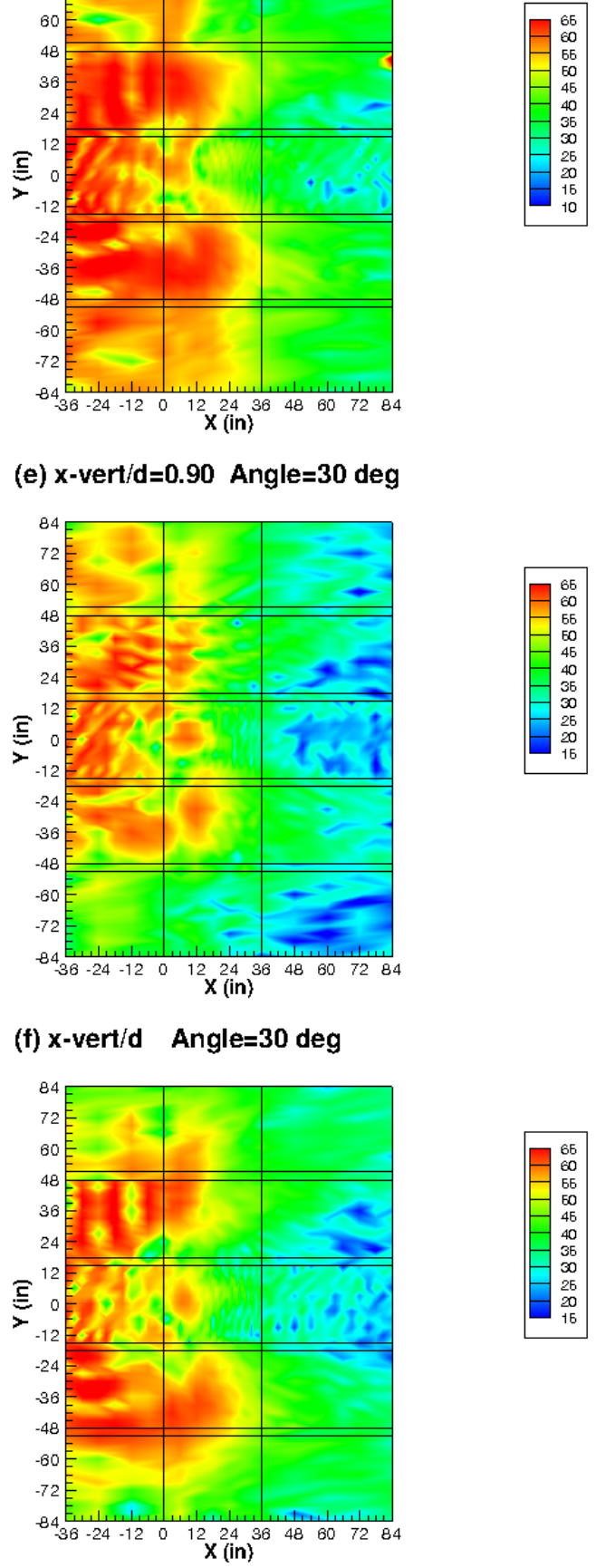
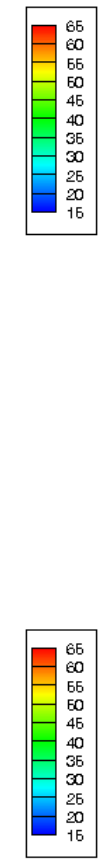

Figure 33.-Far-field SPL contour plot at with verticals mounted. Left column is from isolated exhaust nacelle; right column is nacelle mounted on 2-D wing with trailing edge. $x / d=2.5, \theta=0^{\circ}$, Approach, $2 \mathrm{BPF}, \mathrm{H} 1$ measurement plane, Signal 1. 
Finally, the difference between inlet nacelle/leading edge and exhaust nacelle/trailing edge patterns for the mounted nacelle is illustrated in Figure 34. First by comparing the exhaust and inlet nacelle at the same geometric positions $\left(x / d=2.5, \theta=45^{\circ}\right.$, see Figure 34(a) and (b)) and then observing the effect of moving the inlet nacelle far from the leading edge (Figure 34(c)), as this is a more representative case for the forward portion of the aircraft's radiation (the nacelle sits at the aft of the aircraft as seen in Figure 1). The inlet nacelle appears to slightly reduce the beaming of the principal lobe.

(a) Exhaust $x / d=2.5$

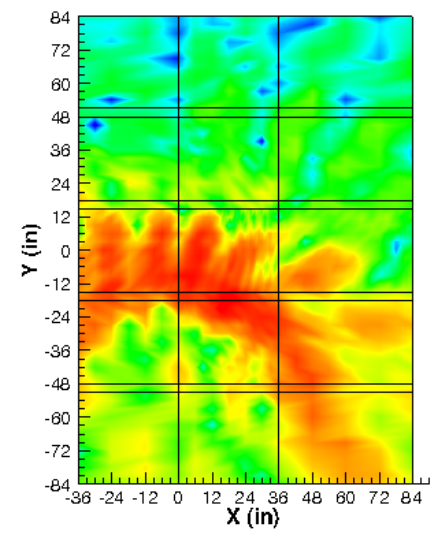

(b) Inlet $x / d=2.5$

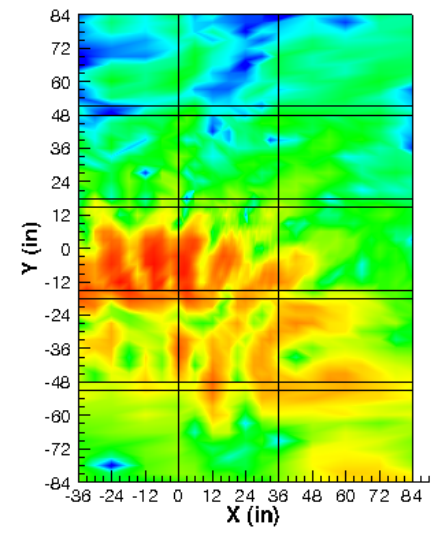

(c) Inlet $x / d=4.0$

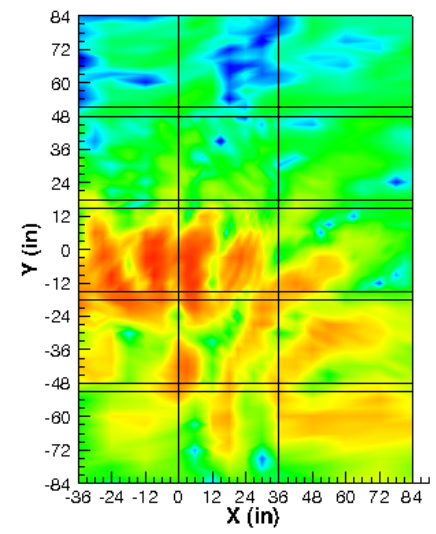

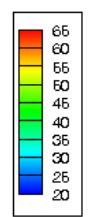
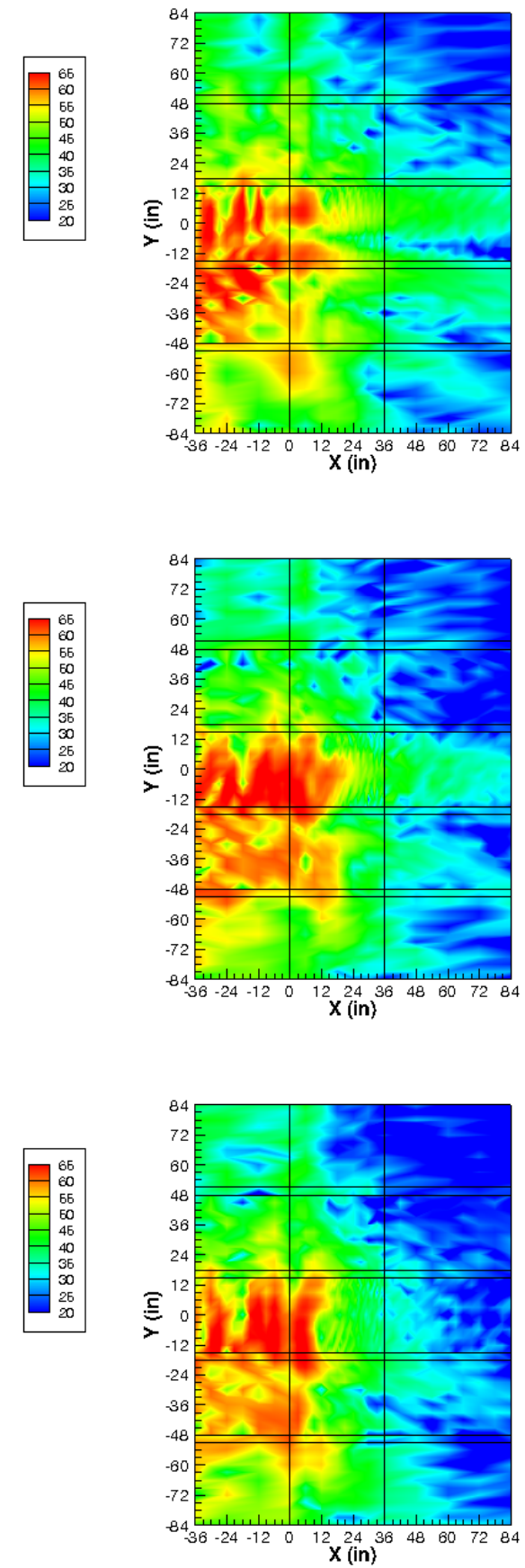

Figure 34.-Far-field SPL contour plot comparing exhaust to inlet (EX, IN, IN). Left column is from isolated inlet nacelle; right column is nacelle mounted on 2-D wing with leading edge. $\theta=45^{\circ}$, Approach, $2 \mathrm{BPF}, \mathrm{H} 1$ measurement plane, Signal 1. 
The power in the measurement plane was computed for each configuration. By comparing an isolated nacelle configuration to the corresponding case with the wall in place, a qualitative value of the shielding can be determined. This computation has several limitations, not the least of which is the limited geometry, but can provide some insight as to trends. Figure 35 to Figure 38 show the shielding achieved for each configuration as defined by this computation. Each plot shows the del-PWL (delta-PWL) (positive number indicates effective shielding) for all four-measurement planes, the three rating points, and the fan harmonics. The overall effect confirmed is that the horizontal planes do not show significant shielding as a result of the acoustic pattern being reflected upward from the wing and contained with-in the measurement plane. The vertical planes show the qualitative shielding as a result of the wing blocking a significant fraction of the radiated acoustics.

Figure 35 shows the change in PWL achieved at various axial positions of the nacelle relative to the trailing edge. It is pretty straightforward and expected observation that as the nacelle is stationed closer to the trailing edge, the effectiveness of the plan-form is reduced slightly ( $x / d$ from 3.14 to 2.0 ) in the vertical planes. At $x / d=3.14$ the range of del-PWL is roughly 15 to $20 \mathrm{~dB}$, at $x / d=2.0$ it is closer to 10 to $15 \mathrm{~dB}$. As the nacelle exhaust plane is positioned even with the trailing edge, or even over hanging ( $x / d$ from 0.0 to -0.5 ) the effectiveness of the wing as a shield is significantly reduced to near $0 \mathrm{~dB}$. There is a significant decrease in the computed shielding value between $x / d=2.5$ and 0.0 , indicating a finer investigation into the axial spacing maybe warranted to achieve an optimum. The horizontal planes show insignificant changes in PWL between the isolated- and mounted-nacelle configurations. This is due to the acoustic radiation being reflected and fully captured by the measurement plane.
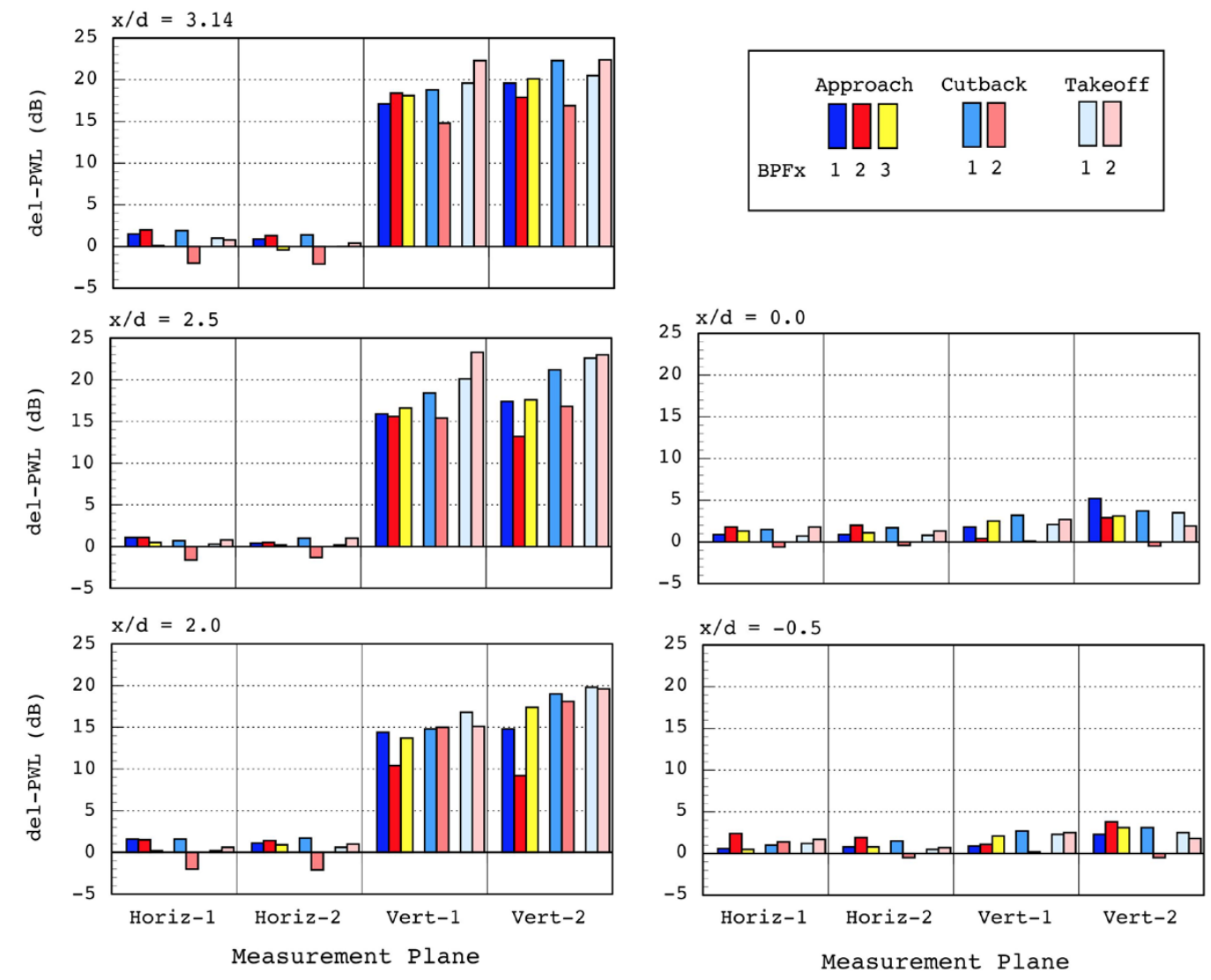

Figure 35.-Computed PWL values for all measurement planes at varying X/D. Exhaust Nacelle, $\mathrm{H} 1$ measurement plane, $\theta=0^{\circ}$, Signal 1 . 
Figure 36 shows the effect of varying the nacelle angle on the change in computed PWL. As the angle of the principal lobe is changed relative to the wing trailing edge the shielding is affected. Depending on the mode and nacelle angle, this lobe angle may beam directly toward the trialing edge, and a resulting increase in the del-PWL is noted as the angle relative to the trailing edge is increased. At $60^{\circ}$ nacelle angle a few decibels of del-PWL occurs in the horizontal plane. At this angle the relative angle between the radiated lobe and the trailing edge is large enough that such that the measurement plane does not capture all of the acoustic reflection, not necessarily a true shielding effect.

Figure 37 shows the changes in shielding as a result of the mounting of the verticals. Subtle differences are noted in the qualitative values, as the effect is more noted in the earlier contour plots. The del-PWL measured in the vertical planes is fairly consistent 15 to $20 \mathrm{~dB}$, similar to the mounted-nacelle with no verticals installed.

Figure 38 compares the exhaust nacelle to inlet nacelle. The data indicate that the rounder leading edge results in higher shielding than the sharp trailing edge, at the same $x / d$ position, perhaps due to less sharp diffraction. Of course as the axial position is increased to be more representative of the distance to the trailing edge, the attenuation from the 2-D plan form is greater, a change from 15 to $20 \mathrm{~dB}$ to 20 to $25 \mathrm{~dB}$.

Limited repeatability and acoustic signal to noise ratio were presented in the earlier paper (Ref. 7) using simpler code validation modes.
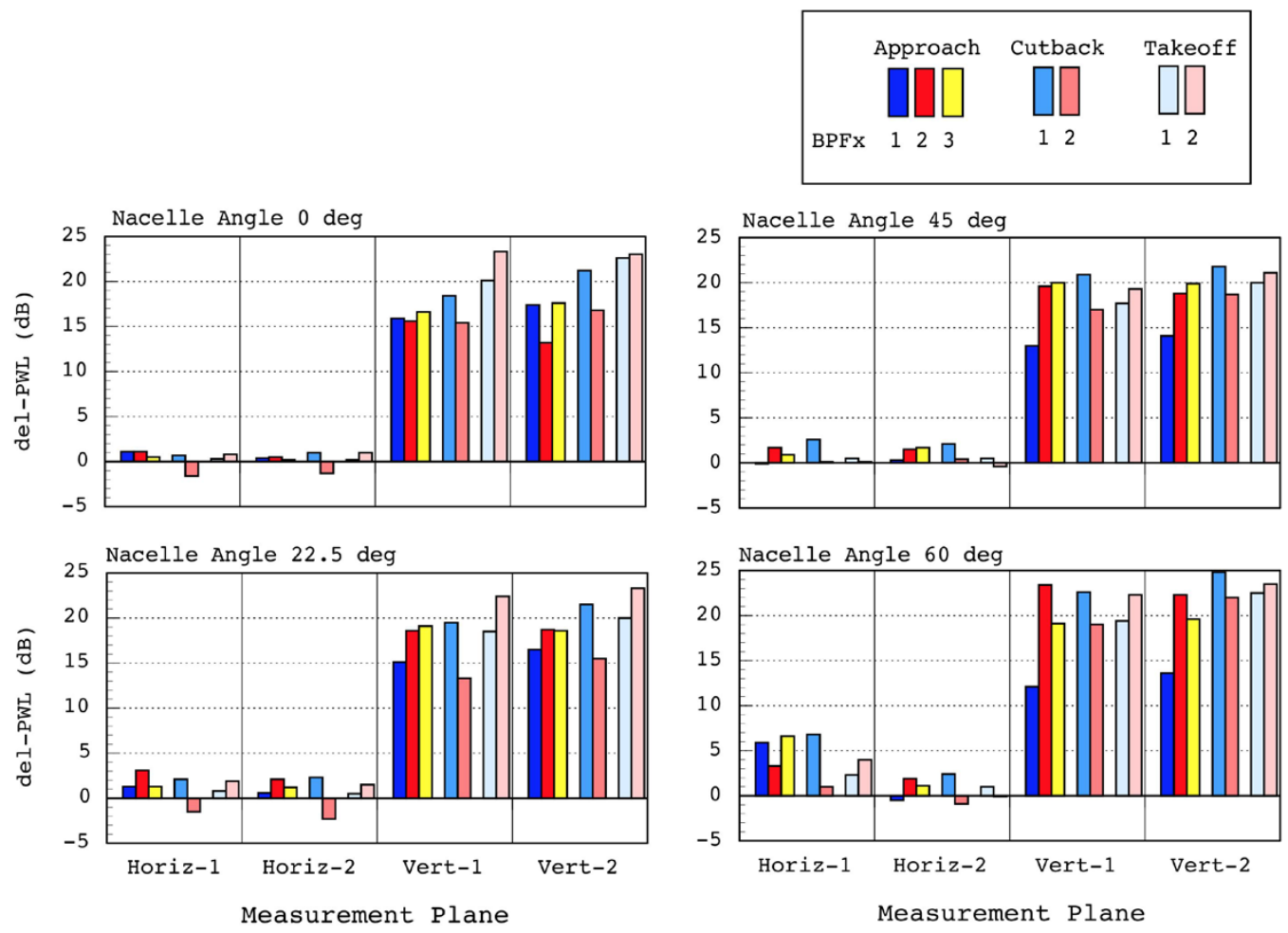

Figure 36.-Computed PWL values for all measurement planes at varying nacelle angle. Exhaust Nacelle, $\mathrm{H} 1$ measurement plane, $x / d=2.5$, Signal 1. 

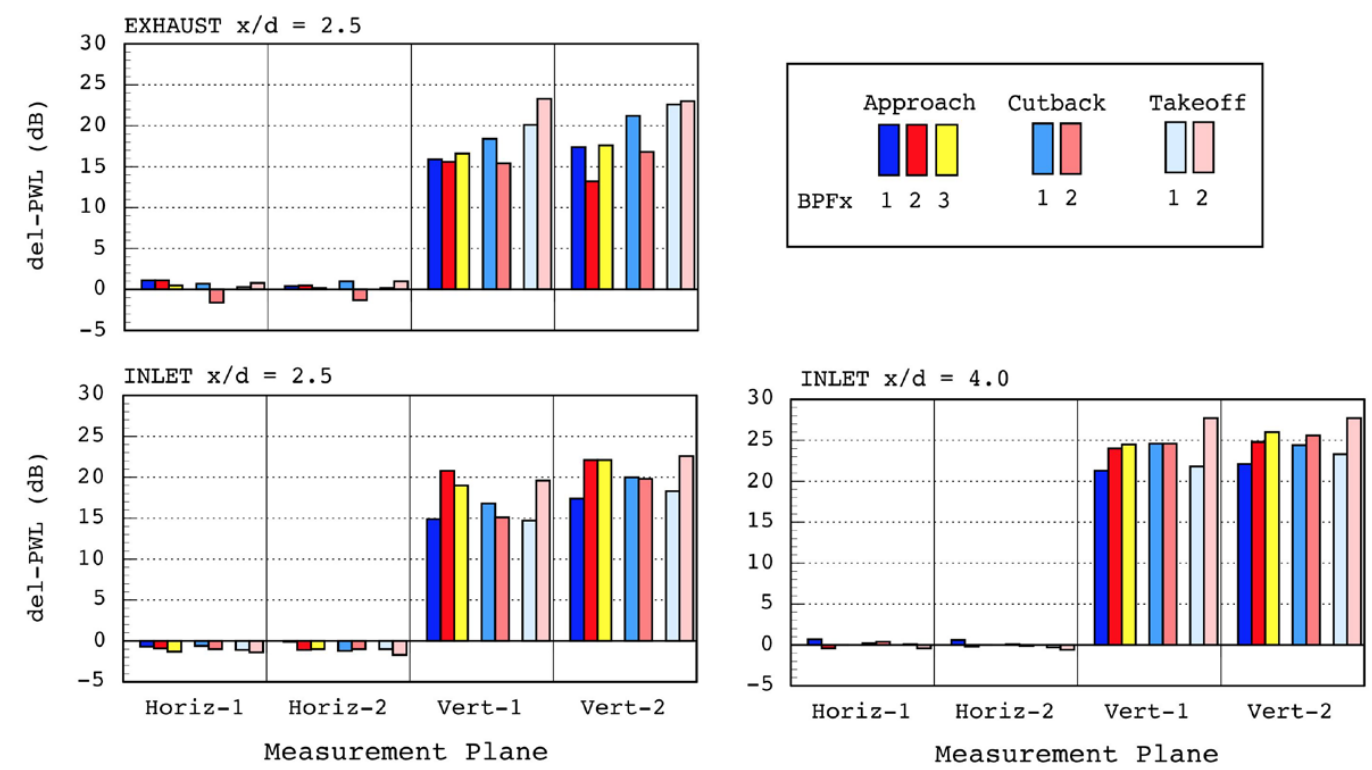

Figure 37.-Computed PWL values for all measurement planes for inlet nacelle. Various nacelle, $\theta=0^{\circ}, x / d=2.5, \mathrm{H} 1$ measurement plane, Signal 1
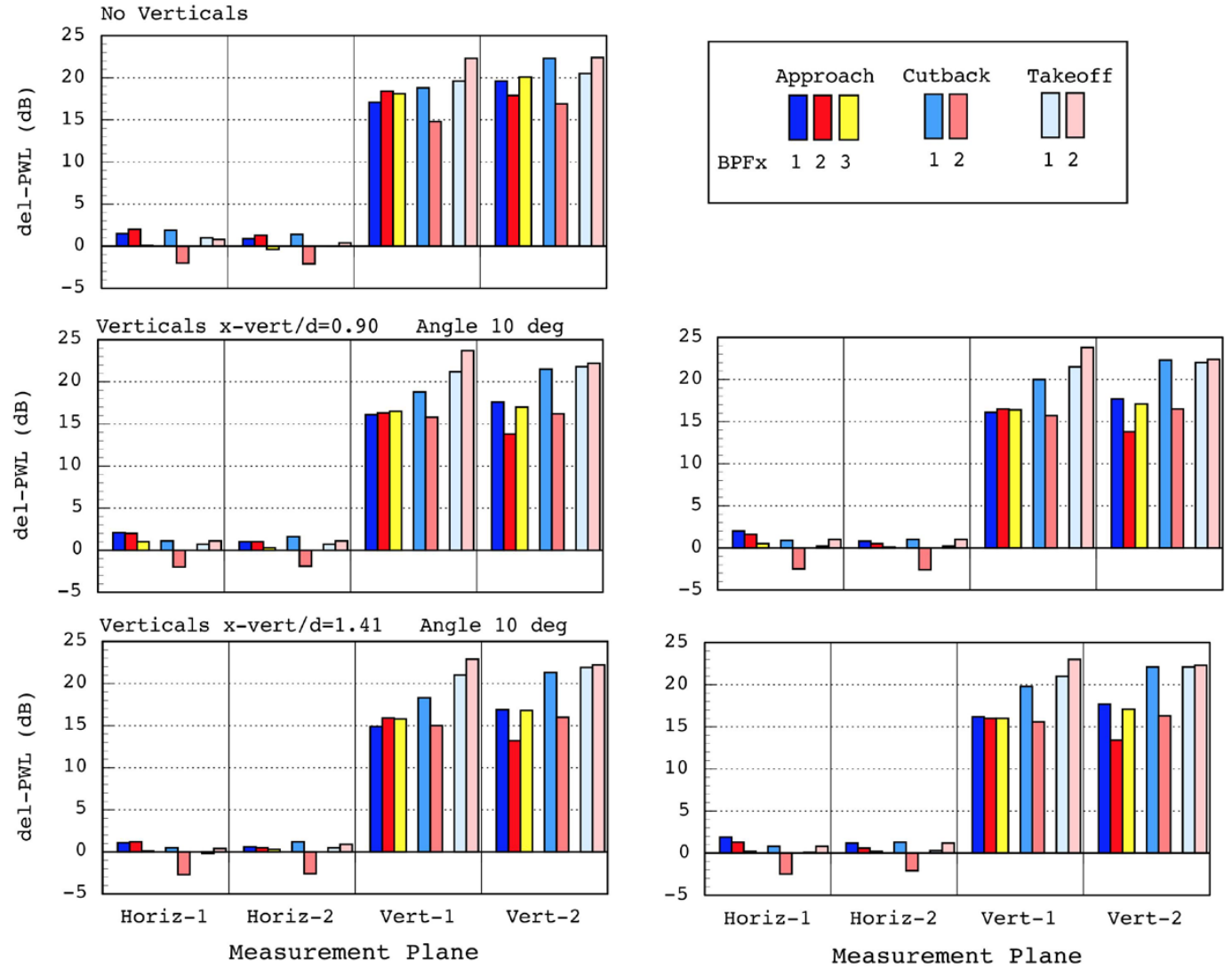

Figure 38.-Computed PWL values for all measurement planes with verticals installed. Exhaust nacelle, $\theta=0^{\circ}, x / d=2.5, \mathrm{H} 1$ measurement plane, Signal 1 . 


\subsection{Conclusion}

The Ultrasonic Configurable Fan Artificial Noise Source test fixture is a configurable fan-mode simulator for scale model testing of engine acoustic propagation and interaction with the airframe. The work herein was a direct follow-on to an earlier work that documented a simpler set of modes. The frequencies, and hence more complex modal structure, reported in this paper were based on the operating points for the engine proposed for the Hybrid Wing Body aircraft. A sample subset of data was presented for illustrative purposes representing the range of cases tested. In-duct modal analysis and external radiation patterns confirm that consistent targeted combinations of frequencies and modal structure can be achieved. Data from in-duct circumferential array microphones and far-field acoustic linear array traverses were acquired. From this data the modal content and far-field power was computed.

A variety of geometric configurations were tested using both an exhaust nacelle/trailing edge and inlet nacelle /leading edge. Nacelle axial location and angle were varied. The effect of vertical stabilizer installation was determined at several positions. Data were acquired for the first three fan harmonics of the rating points at various nacelle angle and axial position on the wing. The shielded/unshielded configurations were compared. Overall the biggest impact on the far field sound power level is the distance between the nacelle acoustic radiation plane and the wing/wall edge.

The more complex signature required for this work required deliberate compromises. These include underspecifying the mode generation and measurement resulting from geometric space constraints. Also, the electronic circuitry utilized for the in-duct microphone had issues with interference due to necessity to utilize low cost microphones. It is believed that these compromises, while providing opportunities for improvement, did not affect the goals of the research - the far-field modal and shielding characteristics are as would be intuitively expected and thus suitable for first-order approximation of the fan tone noise shielding benefits for system study purposes. 


\section{Appendix-Nomenclature}

\section{Acronyms}

\begin{tabular}{|c|c|}
\hline $\mathrm{A} / \mathrm{D} ; \mathrm{D} / \mathrm{A}$ & Analog-to-Digital; Digital-to-Analog \\
\hline ATL & Acoustic Testing Laboratory \\
\hline $\mathrm{BPF}$ & Blade Passing Frequency \\
\hline CVM & Code Validation Modes \\
\hline DADS & Digital Acoustic Data System \\
\hline del-PWL & delta-PWL \\
\hline GUI & Graphical User Interface \\
\hline HWB & Hybrid Wing Body \\
\hline $\mathrm{kS} / \mathrm{s}$ & kilo-samples per second \\
\hline MLS & Maximum Length Sequence \\
\hline $\mathrm{N}+2$ & second generation aircraft beyond current state-of-the-art \\
\hline PWL & power level (computed) \\
\hline SPL & Sound Pressure Level \\
\hline UCFANS & Ultrasonic Configurable Fan Artificial Noise Source \\
\hline \multicolumn{2}{|l|}{ Symbols } \\
\hline$a$ & area \\
\hline$d$ & inlet duct diameter \\
\hline$c$ & speed of sound \\
\hline$f$ & frequency \\
\hline$k$ & wave-number \\
\hline$m$ & circumferential mode order \\
\hline$n$ & radial mode order \\
\hline$p$ & pressure \\
\hline$r$ & radius \\
\hline$x$ & axial distance on model \\
\hline$A$ & axial spacing matrix \\
\hline$B$ & beam-forming matrix \\
\hline$C$ & cross-spectral matrix \\
\hline$H$ & transfer function matrix \\
\hline$J$ & Bessel function-First kind \\
\hline$L$ & sound power \\
\hline$M$ & microphone index \\
\hline$N$ & number of traverse stops \\
\hline$P$ & mode pressure \\
\hline$S$ & steering vector \\
\hline$T$ & transfer matrix \\
\hline$Y$ & Bessel function-Second kind \\
\hline$(\mathrm{X}, \mathrm{Y}, \mathrm{Z})$ & traverse co-ordinate system \\
\hline$\alpha$ & eigenvalue \\
\hline$\beta$ & annular duct weighting coefficient \\
\hline$\theta$ & nacelle angle relative to edge \\
\hline$\delta$ & vertical cant angle \\
\hline$\Delta$ & traverse stop resolution \\
\hline$\eta$ & cut-off ratio \\
\hline$\psi$ & annular duct basis function \\
\hline
\end{tabular}




\section{References}

1. Hill, G.A. and Thomas, R.H., "Challenges and Opportunities for Noise Reduction Through Advanced Aircraft Propulsion Airframe Integration and Configurations," presented at the 8th CEAS Workshop on Aeroacoustics of New Aircraft and Engine Configurations, Budapest, Hungary, Nov. 11-12, 2004.

2. Thomas, R.H., "Subsonic Fixed Wing Project N+2 Noise Goal Summary," presentation at the NASA Acoustics Technical Working Group, December 4-5, 2007, Cleveland, OH.

3. Czech, M.J., Thomas, R.H., and Elkoby, R., "Propulsion Airframe Aeroacoustic Integration Effects for a Hybrid Wing Body Aircraft Configuration," International Journal of Aeroacoustics, Vol. 11, Number 3+4, 2012.

4. Thomas, R.H., Burley, C.L., and Olson, E.D., "Hybrid Wing Body Aircraft System Noise Assessment with Propulsion Airframe Aeroacoustic Experiments," International Journal of Aeroacoustics, Vol. 11, Number 3+4, 2012.

5. "Acoustic Prediction Methodology and Test Validation for an Efficient Low-Noise Hybrid Wing Body Subsonic Transport," Final Report under NASA Contract Number NNL07AA54C, September 2008.

6. HWB N+2 Phase II Final Report, NASA ARMD Subsonic Fixed Wing Project, "Acoustic Prediction Methodology and Test Validation for an Efficient Low-Noise Hybrid Wing Body Subsonic Transport," NASA Contract Number NNL07AA54C, Final Report, February 2011.

7. Daniel L. Sutliff, Clifford A. Brown, and Bruce E. Walker, "Hybrid Wing Body Shielding Studies Using an Ultrasonic Configurable Fan Artificial Noise Source," NASA/TM-2012-217685 also AIAA-2012-2076, Nov 2012.

8. Beth Cooper, Alan Eckel, Trent Butcher, and David Nelson, "New Acoustical Testing Laboratory at NASA," Sound \& Vibration. Dec. 2000: 10-18.

9. Schroeder, M.R., Number Theory in Science and Communication, Springer-Verlag 1984.

10. http://www.wildlife-sound.org/equipment/technote/micdesigns/ultrasonic.html, accessed 27-October, 2011. 



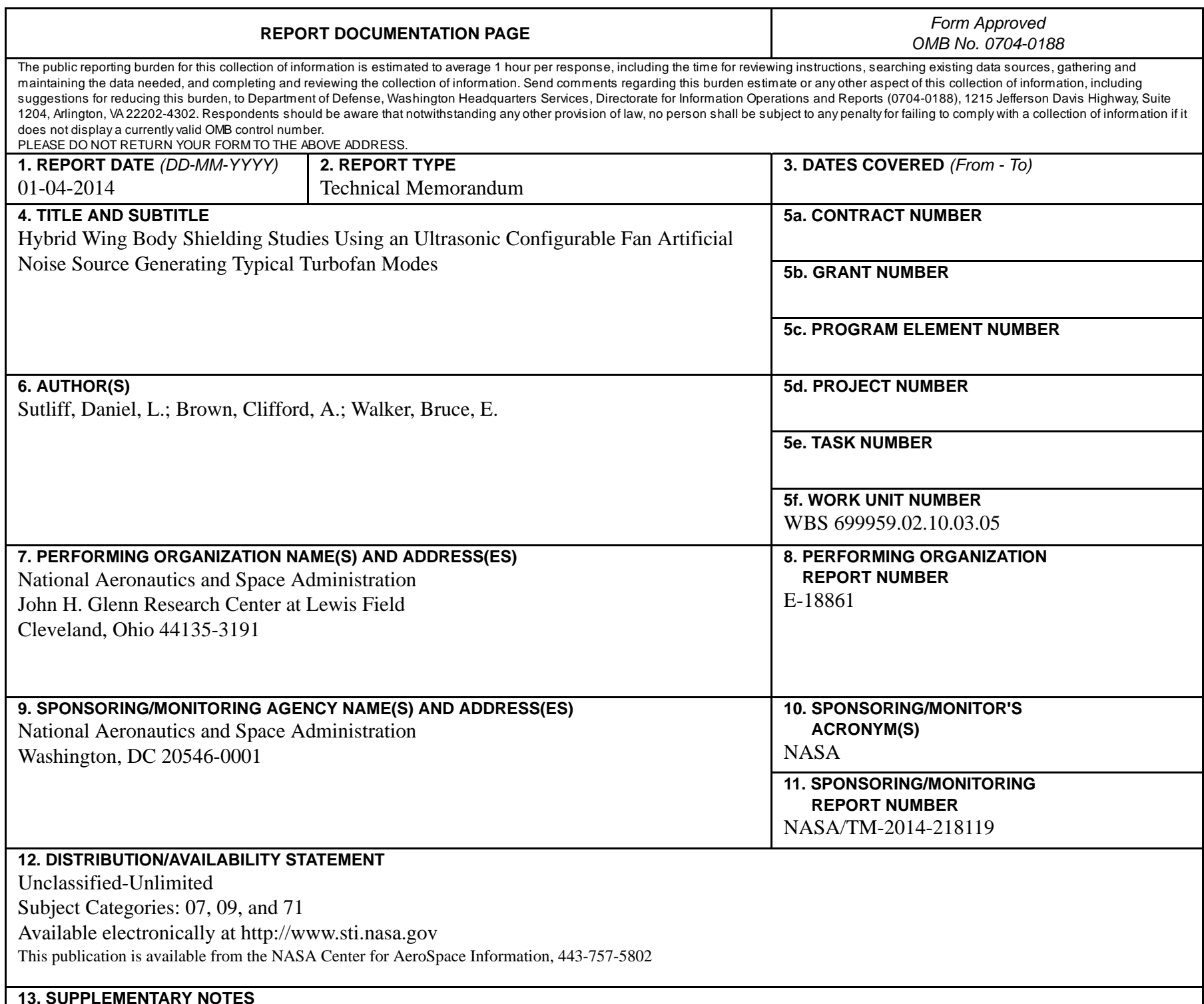

\section{SUPPLEMENTARY NOTES}

\section{ABSTRACT}

An Ultrasonic Configurable Fan Artificial Noise Source (UCFANS) was designed, built, and tested in support of the NASA Langley Research Center's 14- by 22-ft wind tunnel test of the Hybrid Wing Body (HWB) full 3-D 5.8 percent scale model. The UCFANS is a 5.8 percent rapid prototype scale model of a high-bypass turbofan engine that can generate the tonal signature of proposed engines using artificial sources (no flow). The purpose of the test was to provide an estimate of the acoustic shielding benefits possible from mounting the engine on the upper surface of an HWB aircraft using the projected signature of the engine currently proposed for the HWB. The modal structures at the rating points were generated from inlet and exhaust nacelle configurations--a flat plate model was used as the shielding surface and vertical control surfaces with correct plan form shapes were also tested to determine their additional impact on shielding. Radiated acoustic data were acquired from a traversing linear array of 13 microphones, spanning 36 in. Two planes perpendicular, and two planes parallel, to the axis of the nacelle were acquired from the array sweep. In each plane the linear array traversed four sweeps, for a total span of 168 in. acquired. The resolution of the sweep is variable, so that points closer to the model are taken at a higher resolution. Contour plots of Sound Pressure Levels, and integrated Power Levels, from nacelle alone and shielded configurations are presented in this paper; as well as the in-duct mode power levels.

\section{SUBJECT TERMS}

Aeroacoustics; Ducted fans; Fan noise; Test

\begin{tabular}{|l|l|l|l|l|l|}
\hline \multicolumn{2}{|l|}{ 16. SECURITY CLASSIFICATION OF: } & 17. LIMITATION OF & $\begin{array}{l}\text { 18. NUMBER } \\
\text { ABSTRACT }\end{array}$ & $\begin{array}{l}\text { 19a. NAME OF RESPONSIBLE PERSON } \\
\text { OF }\end{array}$ \\
\cline { 1 - 2 } $\begin{array}{l}\text { a. REPORT } \\
U\end{array}$ & $\begin{array}{l}\text { b. ABSTRACT } \\
\text { S }\end{array}$ & $\begin{array}{l}\text { c. THIS PAGE } \\
\text { U }\end{array}$ & UU & 52 & $\begin{array}{l}\text { 19b. TELEPHONE NUMBER (include area code) } \\
443-757-5802\end{array}$ \\
\hline
\end{tabular}



